\title{
Das Institut für Deutsche Sprache im Jahre 2001
}

\section{Inhalt}

1. Vorbemerkungen

2. Mitarbeiter und Arbeiten der Abteilungen und Arbeitsstellen

3. Tagungen, Kolloquien und Vorträge externer Wissenschaftler am IDS

4. Lehraufträge und Vorträge von IDS-Mitarbeitern

5. Im Berichtsjahr erschienene Publikationen von IDS-Mitarbeitern

6. Kontakte des IDS zu anderen Institutionen, Studienaufenthalte und Besuche in- und ausländischer Wissenschaftler am IDS, Praktika, Besuchergruppen

7. Gremien des Instituts für Deutsche Sprache

8. Besondere Nachrichten

9. Personalstärke, Anschrift, finanzielle Angaben

10. Veröffentlichungen im Jahre 2001

\section{Vorbemerkungen}

\subsection{Aufgaben und Ziele}

Das Institut für Deutsche Sprache (IDS) wurde 1964 in Mannheim gegründet und hat hier seitdem seinen Standort. Es ist die zentrale staatlich geförderte Einrichtung zur Erforschung und Dokumentation der deutschen Sprache in ihrem gegenwärtigen Gebrauch und ihrer neueren Geschichte. Als Mitglied der Wissenschaftsgemeinschaft Gottfried Wilhelm Leibniz (WGL) wird das Institut finanziell je zur Hälfte vom Bund und vom Land BadenWürttemberg getragen. Hinzu kommen in wechselndem Umfang Mittel von forschungsfördernden Organisationen wie der Deutschen Forschungsgemeinschaft und der Volkswagen-Stiftung. Förderung erfährt das IDS auch von der Stadt Mannheim und dem Verein der Freunde des Instituts für Deutsche Sprache e. V. In seinen laufenden wissenschaftlichen Arbeiten und seiner Forschungsplanung orientiert sich das Institut an folgenden Richtlinien (i.d.F. vom 5.11.1998), die seinen generellen Auftrag näher bestimmen:

Richtlinien für die wissenschaftliche Arbeit des Instituts für Deutsche Sprache (IDS)

(1) Das IDS hat die Aufgabe, die deutsche Sprache in ihrem gegenwärtigen Gebrauch und in ihrer neueren Geschichte wissenschaftlich zu erforschen und zu dokumentieren. Untersucht wird die deutsche Sprache in 
ihren verschiedenen Ausprägungen. Fragen der Sprachentwicklung, der Sprachnormung, der Sprachkritik, des Sprachkontakts und des Spracherwerbs werden berücksichtigt und zwar auch im europäischen Kontext.

(2) Das IDS verfolgt in erster Linie längerfristige Vorhaben, die umfangreiche Datensammlungen, eine größere apparative Ausstattung und eine Arbeit in Forschungsgruppen erforderlich machen. Kleinere befristete Projekte sind den größeren Forschungsvorhaben zugeordnet. Bei der Vorbereitung und Durchführung seiner Vorhaben arbeitet das IDS mit Hochschulinstituten und mit anderen Forschungseinrichtungen zusammen.

(3) Ziele der Untersuchungen sind übergreifende Darstellungen des Sprachsystems und der Sprachverwendung sowie vergleichende Beschreibungen innersprachlicher Varianten und Darstellungen des Deutschen im Vergleich mit anderen Sprachen.

(4) Das IDS nutzt die Möglichkeiten der Datenverarbeitung für seine Forschungen. Es baut maschinenlesbare Korpora zum geschriebenen und gesprochenen Deutsch auf, nutzt zu deren Auswertung Ergebnisse und Methoden der Computerlinguistik und entwickelt auch selbst Verfahren zur Korpuserschließung. Die Korpora sind Grundlage der linguistischen Forschungen des IDS, können aber auch von externen Partnern für wissenschaftliche Zwecke verwendet werden.

(5) Die Forschungsergebnisse des IDS stehen als linguistische Grundlagen für den Unterricht in Deutsch als Muttersprache und als Fremdsprache, für die Sprachberatung, die Sprachnormung, die sprachliche Informationsverarbeitung und die Sprachtherapie zur Verfügung.

(6) Das IDS leistet wissenschaftliche Dienste für seine eigenen Forschungsvorhaben (DV-Unterstützung, Bibliothek, Textsammlungen, Dokumentationen, Archive). Die Dienste stehen auch Forschern und Forschergruppen außerhalb des IDS zur Verfügung.

(7) Aufgabe des IDS ist auch, Verbindungen zwischen der sprachgermanistischen Forschung im In- und Ausland herzustellen und zu erhalten. Diesem Zweck dient vor allem die Veranstaltung von Fachtagungen und Kolloquien.

Neben der Direktion und der Verwaltung besteht das Institut aus folgenden Abteilungen und zentralen Arbeitsstellen:

\section{Abteilung Grammatik}

In der Abteilung werden die grammatischen Strukturen des Deutschen erfasst und beschrieben, u. a. auch im Vergleich mit anderen Sprachen.

\section{Abteilung Lexik}

Bearbeitet werden lexikologische und lexikografische Aufgaben; hierzu untersucht die Abteilung definierte lexikalische Bereiche und erstellt möglichst umfassende Dokumentationen des deutschen Wortschatzes. 


\section{Abteilung Pragmatik/Sprachverwendung}

Forschungsgegenstände der Abteilung sind sprachliches Handeln und sprachliche Variabilität, d.h. die Ausprägung und Entwicklung von Sprachunterschieden. Besondere Berücksichtigung findet der mündliche Sprachgebrauch.

\section{Öffentlichkeitsarbeit und Dokumentation, Bibliothek}

In der Arbeitsstelle sind die Bereiche Öffentlichkeitsarbeit und Presse, Publikationswesen, Dokumentation und Bibliothek zusammengefasst.

\section{Zentrale Datenverarbeitungsdienste (ZDV)}

Die ZDV unterstützt die computerbasierten Arbeiten der Abteilungen durch die Bereitstellung und Pflege der erforderlichen Hard- und Software.

\subsection{Allgemeines und Bemerkenswertes}

Herausragendes Ereignis im Berichtsjahr war wieder die Jahrestagung, die 37. seit der Gründung des IDS, diesmal zu dem Thema: „Sprache - Recht - Öffentlichkeit". Angesichts dieses Themas mit multidisziplinären Implikationen ist nicht weiter verwunderlich, dass neben mehreren Hundert Germanisten aus dem In- und Ausland auch viele Juristen an der Tagung teilnahmen. Näheres zu den Vorträgen und Diskussionen findet sich in Abschnitt 3.1.

Die Forschungsarbeiten und wissenschaftlichen Dienste des Instituts orientierten sich an dem seit 1998 geltenden mittelfristigen Arbeitsplan, der aber aufgrund personeller und sachlicher Neuerungen in einigen Teilbereichen modifiziert wurde. Über die einzelnen Projekte und Serviceleistungen wird aus den Forschungsabteilungen und wissenschaftlichen Arbeitsstellen in den folgenden Abschnitten berichtet.

Neben den laufenden Arbeiten, zu der auch die aufgabenspezifische Vorlesungs- und Vortragstätigkeit der Mitarbeiter gehörte, war das Institut während des Berichtsjahrs besonders häufig in die öffentliche sprachpolitische Diskussion einbezogen. Das mehrere Jahre lang vorherrschende Thema "Rechtschreibreform" wurde durch die Themen „Anglizismen im Deutschen" und ,Mehrsprachigkeit"abgelöst. Mehrere Wissenschaftlerinnen und Wissenschaftler des IDS wurden wiederholt zu Interviews der verschieden Medien aufgefordert oder zu Podiumsgesprächen und Vorträgen über diese dominanten Themen eingeladen. An verschiedenen Veranstaltungen zum „Europäischen Jahr der Sprachen" waren auch Vertreter des IDS beteiligt. Mit seinem Memorandum „Politik für die deutsche Sprache" (abgedruckt im Sprachreport 2/2001, S. 8-10), das auch mehreren staatlichen Stellen übermittelt wurde, setzte sich der Institutsdirektor unter anderem für die verstärkte schulische Förderung der deutschen Sprache und der Mehrsprachigkeit ein.

Die wissenschaftlichen Außenkontakte des Instituts wurden weiter entwickelt. Hierzu gehörte der Abschluss eines Kooperationsvertrags mit der 
Philologischen Fakultät der Lomonosov-Universität in Moskau, der am 22.6.2001 unterschrieben wurde. Er soll besonders die Zusammenarbeit im Bereich der linguistischen Medienforschung erleichtern.

Der Wissenschaftliche Beirat des IDS setzte im Berichtsjahr den Dreijahresturnus seiner Evaluation der Abteilungen des Instituts fort. Abgeschlossen wurde mit insgesamt gutem Ergebnis die Bewertung der Abteilung Grammatik. Als zunächst letzte der drei Abteilungen wurde am 22./ 23. November die Abteilung Lexik , begangen'. Eine zusammenfassende Bewertung legt der Beirat den anderen Organen des Instituts im Frühjahr 2002 vor.

Über die vielen weiteren Aktivitäten im Institut und anderswo, an denen Wissenschaftlerinnen und Wissenschaftler des IDS beteiligt waren, und auch über die im Berichtsjahr erschienenen Publikationen informieren die folgenden Kapitel.

\section{Mitarbeiter und Arbeiten der Abteilungen und Arbeitsstellen}

\subsection{Grammatik}

\section{Abteilungsleiterin:}

Prof. Dr. Gisela Zifonun

\section{Wissenschaftliche Mitarbeiterinnen und Mitarbeiter:}

Prof. Dr. Joachim Ballweg - PD Dr. Hardarik Blühdorn (seit 1.4.2001) - Dr. Eva Breindl-Hiller - Dr. Elke Donalies - Helmut Frosch - Lutz Gunkel (seit 1.3. 2001) - Dr. Klaus Heller - Dr. Ursula Hoberg (bis 31.12.2001) - Dr. Marek Konopka (seit 1.4. 2001) - Jaqueline Kubczak - PD Dr. Gereon Müller (seit 1.4.2001) - Dr. Renate Pasch - Dr. Horst Schwinn (seit 1.4.2001) Helmut Schumacher - Dr. Angelika Storrer (bis 30.9. 2001 beurlaubt)- Prof. Dr. Bruno Strecker - Dr. h. c. Klaus Vorderwülbecke - Dr. Ulrich Hermann Waßner

\section{Sekretärinnen: Ruth Maurer - Karin Laton}

Die Abteilung Grammatik hat folgende Zielsetzungen:

a. Sie erforscht und dokumentiert die grammatischen Strukturen der deutschen Gegenwartssprache, auch im Vergleich mit anderen Sprachen und in sprachtypologischer Perspektive.

b. Sie erschließt grammatisches Wissen für ein sprachinteressiertes Publikum durch ein grammatisches Informationssystem mit angegliedertem Lernsystem.

Damit sucht die Abteilung einerseits ihrem im engeren Sinne wissenschaftlichen Auftrag gerecht zu werden, andererseits aber will sie eine breitere Öffentlichkeit für die Sache der Grammatik als des zentralen sprachlichen Kenntnissystems interessieren und gewinnen. Für die Abteilungsarbeit sind derzeit folgende Schwerpunkte gesetzt: 
- Grammatik und neue Medien, mit dem hypermedialen grammatischen Informationssystems GRAMMIS, den Drittmittelprojekten „ProGr@mm“ und „Hypertextualisierung und Textgrammatik (HyTex)“.

- Handbuch der deutschen Konnektoren

- Grammatik des Deutschen im europäischen Vergleich

Daneben wird noch das Projekt „Valenzlexikon deutscher Verben VALBU“ weitergeführt. Die Geschäftsstelle der Zwischenstaatlichen Kommission für deutsche Rechtschreibung ist als Arbeitsbereich mit Daueraufgaben ebenfalls der Abteilung Grammatik zugeordnet. Projektarbeiten der Arbeitsstelle „Graphie und Orthographie“ (bis zum Jahr 2000) konnten trotz des altersbedingten Ausscheidens des Bearbeiters in reduziertem Umfang weitergeführt werden.

\section{GRAMMIS}

Mit dem Projekt GRAMMIS steht die Entwicklung eines umfassenden multimedialen, elektronisch vernetzten Informationssystems zur deutschen Grammatik, auf das weltweit über das Internet zugegriffen werden kann, auf dem Programm. (http://hypermedia.ids-mannheim.de/grammis) Das grammatische Informationssystem soll einem breiten sprachinteressierten Publikum grammatisches Wissen in verständlicher und leicht zugänglicher Weise vermitteln. Es wird unter Nutzung moderner Hyper- und Multi-Media-Techniken in mehreren Stufen rechnergestützt entwickelt. Alle Informationseinheiten sind in XML (Extensible Markup Language) verfasst und damit unabhängig von jeweils verfügbaren Computerplattformen zugänglich. Das System setzt sich aus mehreren Komponenten zusammen, von denen zunächst folgende bearbeitet werden, weil sie den informatorischen Kern des Systems bilden:

- Systematische Grammatik (Kurzform auf GRAMMIS WWW-Seiten: Grammatik)

- Glossar grammatischer Termini (Kurzform auf GRAMMIS WWW-Seiten: Glossar)

- Grammatisches Wörterbuch (Kurzform auf GRAMMIS WWW-Seiten: Wörterbuch)

- Bibliografie zur deutschen Grammatik

Um das Auffinden gewünschter Informationen für nicht sprachwissenschaftlich vorgebildete Nutzer zu erleichtern, sollen als weitere Komponenten hinzukommen:

- Hauptschwierigkeiten der deutschen Grammatik

- Häufig gestellte Fragen zur deutschen Grammatik

- ein Suchleitsystem

Wissenschaftliche Grundlage von GRAMMIS ist in weiten Teilen (mit Ausnahme der Einheit, Wortbildung") die am IDS verfasste "Grammatik der 
deutschen Sprache“ (Zifonun et al. 1997); die Informationseinheiten werden jedoch nicht einfach in ein neues Medium umgesetzt, sondern so aufbereitet, dass auch ein weniger fachkundiges Publikum angesprochen werden kann. Soweit möglich werden aktuelle Forschungsergebnisse anderer Abteilungsprojekte einbezogen.

Im Zentrum der Arbeit an GRAMMIS stand im Berichtsjahr die Entwicklung weiterer Informationseinheiten zu den Bereichen, Systematische Grammatik', ,Glossar grammatischer Termini' (ca. 280 Einheiten), ,Grammatisches Wörterbuch' sowie, auf technischer Seite, die Optimierung der Benutzerschnittstelle. Fertig gestellt wurden im Berichtsjahr - soweit dies bei einem Hypertext festgehalten werden kann - die Einheiten,Wortbildung', ,Wortstellung', ,Präpositionalphrasen', ,Komplemente' und ,Supplemente'. Fortgeschritten sind die Arbeiten in den Bereichen ,Konnektoren', ,Nominalphrasen' und ,Adverbialia'.

Im Bereich ,Bibliografie zur deutschen Grammatik‘ werden ständig Neuerscheinungen und - Zug um Zug - Arbeiten aus den Jahren 1995 bis 1998 aufgenommen, die zum Zeitpunkt der Übernahme der Daten durch das IDS nur unzureichend erfasst waren.

(Verantwortlich: Bruno Strecker)

\section{ProGr@mm}

Seit dem 1. April 2001 gibt es ein neues Projekt in der Abteilung Grammatik: ProGr@mm. Das Akronym steht für Propädeutische Grammatik und ist als ein interaktives Lernsystem das hochuldidaktische Kind von GRAMMIS.

ProGr@mm stellt primär einen Grundkurs zur deutschen Grammatik für die universitäre Lehre dar. Modulare Einheiten der Grammatik werden als Hypertext vernetzt und unter Nutzung neuer Medien und Techniken - teilweise multimedial - aufbereitet und im Internet zur Verfugung gestellt. Die GRAMMIS-Komponenten ,Terminologisches Wörterbuch', ,Grammatisches Wörterbuch', ,Rechtschreibwörterbuch“ und ,Bibliografie zur deutschen Grammatik' werden auch von ProGr@mm genutzt. Der Kern von ProGr@mm, die ,Propädeutische Grammatik', ist inhaltlich orientiert an der ,Systematischen Grammatik' von GRAMMIS und dadurch auch an der „Grammatik der deutschen Sprache“. Für die ,Propädeutische Grammatik“ werden sowohl eigene Texte entwickelt als auch vorhandene GRAMMISTexte für die didaktische Zielsetzung bearbeitet. Sie enthält darüber hinaus geführte Touren durch den Grundkurs. Zu einem späteren Zeitpunkt wird ProGr@mm ,Seminarbausteine` enthalten, die einzelne Bereiche der Grammatik vertiefen und in thematischen Grammatikseminaren an den Hochschulen genutzt werden können.

Im Berichtszeitraum wurden das Konzept für ProGr@mm entwickelt, die technische Voraussetzung für das Projekt geschaffen und die inhaltlichen Bereiche ,Komplemente', ,Supplemente', ,Tempussystem ' und ,Wortarten' und die dazugehörenden Übungs- und Kontrollaufgaben fertig gestellt. 
Die schon entwickelten Teile von ProGr@mm werden im Wintersemester 2001/02 im Proseminar „Grundkurs Grammatik“ (Prof. Gisela Zifonun) an der Universität Mannheim erprobt und evaluiert.

ProGr@mm ist ein Drittmittelprojekt, das vom Bundesministerium für Bildung und Forschung über drei Jahre im Rahmen des Förderungsprogramms „Neue Medien in der Bildung“ finanziert wird. Innerhalb des IDS bildet Progr@mm zusammen mit GAIS (GesprächsAnalytisches InformationsSystem) aus der Abteilung Pragmatik das abteilungsübergreifende Projekt „Korpustechnologie und linguistische Informationssysteme des IDS“. In beiden Projekten kooperiert die Abteilung unter „PortaLingua“, dem Lern- und Studienportal für Sprach- und Kommunikationswissenschaften, mit Projektgruppen mehrerer Universitäten.

(Verantwortlich: Horst Schwinn)

\section{Hypertextualisierung und Textgrammatik (HyTex)}

Seit November 2001 wird im Projekt HyTex („Hypertextualisierung und Textgrammatik") erforscht, wie die Annotation textgrammatischer Strukturen durch XML-Markup die automatische Konversion sequentiell organisierter Dokumente in Hypertextdokumente leiten und unterstützen kann (vgl. www.hytex.info). Basierend auf theoretischen Überlegungen zur Hypertextkohärenz werden Strategien entwickelt, implementiert und an einem textgrammatisch annotierten Fachtextkorpus evaluiert. Die textgrammatische Annotation nutzt Kategorien aus der „Grammatik der deutschen Sprache“ sowie aus den Projekten „Grammatik des Deutschen im europäischen Vergleich" und „Handbuch der deutschen Konnektoren“. Das Projekt wird als Teil der verteilten Forschergruppe „Texttechnologische Informationsmodellierung" (vgl. www.text-technology.de) von der DFG finanziert.

(Verantwortlich: Angelika Storrer)

\section{Handbuch der deutschen Konnektoren}

Das „Handbuch der deutschen Konnektoren“ beschreibt ca. 300 Wortschatzeinheiten des Deutschen, die inhaltliche Relationen zwischen Sätzen herstellen. Darunter fallen Einheiten wie und, denn und als, die traditionell als Konjunktionen zusammengefasst werden, aber auch viele Adverbien wie dann oder unterdessen und Partikeln wie deshalb, doch, ja oder allein, die in Grammatiken als Konjunktionaladverbien, Abtönungspartikeln oder Fokuspartikeln beschrieben werden. In einem ersten Teil werden die linguistischen Grundlagen der Beschreibung dargestellt, das Verfahren der syntaktischen Subklassenbildung erläutert und es werden die formalen Gebrauchsbedingungen der einzelnen syntaktischen Klassen beschrieben. Semantik und Pragmatik der Konnektoren werden in einem zweiten Teil ausführlich behandelt. Parallel zur Druckversion wird eine Hypertextversion des Handbuchs erarbeitet, die in das elektronische grammatische Informationssystem GRAMMIS integriert wird. 
Im Berichtsjahr wurde an der Druckfassung des ersten Teils des Handbuches, an der semantischen Klassifikation der Konnektoren und an der Erweiterung der Hypertextversion gearbeitet.

Begleitend zu den Arbeiten am Handbuch wird eine Bibliografie erstellt, von der ein einschlägiger Auszug mit derzeit ca. 1.300 Titeln in der Form einer verschlagworteten, abfragbaren Bibliografie-Datenbank auf den WWWSeiten des IDS öffentlich zugänglich gemacht wurde. (www.ids-mannheim.de/gra/konnektoren/)

(Verantwortlich: Renate Pasch)

\section{Grammatik des Deutschen im europäischen Vergleich (GDE)}

Das Ende 1998 begonnene Projekt ,Grammatik des Deutschen im europäischen Vergleich" konnte nach Klärung konzeptioneller Fragen und mit personeller Verstärkung in seine eigentliche Arbeitsphase eintreten. Mit dem Vorhaben soll eine Profilierung der grammatischen Eigenschaften des Deutschen vor dem Hintergrund der entsprechenden Optionen in anderen europäischen Sprachen erreicht werden. Wir versprechen uns auch eine Verbesserung der wissenschaftlichen Grundlagen für die Grammatikvermittlung im Bereich „Deutsch als Fremdsprache“. Nicht zuletzt soll das Projekt auf dem Wege eines besseren Verständnisses sprachstruktureller Gemeinsamkeiten und Kontraste Zugang zu dem kulturellen Wert der Sprachenvielfalt im zusammenwachsenden Europa vermitteln. Dabei ist eine eingehendere Kontrastierung mit europäischen Sprachen wie Englisch, Französisch, Polnisch sowie Ungarisch und eine flexible Einbeziehung anderer Sprachen bei Beschreibungsbedarf geplant.

Im Berichtsjahr wurden in der Reihe amades zwei „Studien zur Grammatik des Deutschen im europäischen Vergleich“ veröffentlicht: „Der Relativsatz“ und „Das Pronomen Teil I: Überblick und Personalpronomen“. Dabei wurde ein einheitliches Darstellungsformat mit den Schwerpunkten ,Typologie', ,Belegung der Varianzparameter in den Kontrastsprachen “ und ,Das Deutsche' zugrunde gelegt. Die Projektgruppe erhofft sich durch diese projektbegleitende Publikation erster Ergebnisse einen intensiven Austausch mit der Fachöffentlichkeit, die der für 2005 geplanten Veröffentlichung des Bandes „Grammatik des Deutschen im europäischen Vergleich" zugute kommen soll. Weitere Arbeitsschwerpunkte lagen im Bereich der morphologischen Kategorien Genus, Kasus und Numerus sowie bei den Determinativen. Zur Problematik der morphologischen Kategorien im deutschen Nominal und der Paradigmenbildung wurden Lösungsvorschläge erarbeitet, der Fachöffentlichkeit in Vorträgen vorgestellt und projektbegleitend veröffentlicht.

(Verantwortlich: Gisela Zifonun)

\section{Valenzlexikon deutscher Verben (VALBU)}

Die Arbeiten zur Fertigstellung des ,Valenzlexikons deutscher Verben“ (Projekt VALBU) wurden fortgesetzt. Das Wörterbuch ist primär für die Verwen- 
dung im Bereich Deutsch als Fremdsprache konzipiert und enthält neben den Informationen zur Valenz genaue morphosyntaktische und semantische Erläuterungen zum Gebrauch der Verben sowie Hinweise zu Wortbildung, Stil, und Phraseologie. Die Strukturbeschreibungen sind durch zahlreiche, überwiegend beleggestützte Verwendungsbeispiele illustriert.

Die Überarbeitung und Endredigierung der 630 Wortartikel wurde abgeschlossen. Gelöst wurden die Probleme des Layouts für die Druckfassung des sehr umfangreichen Manuskripts. Für die Einleitung in das Wörterbuch und die weiteren Rahmentexte liegen Entwürfe vor.

Die Zusammenarbeit mit den ausländischen Partnern, die zweisprachige Versionen von VALBU entwickeln oder verwandte kontrastive Valenzwörterbücher ausarbeiten, konzentrierte sich auf die Unterstützung der japanischen und slowakischen Arbeiten zur kontrastiven Verbvalenz. Fortgesetzt wurde auch die Zusammenarbeit mit dem ungarischen Projekt zur Nominalvalenz und der belgischen CONTRAGRAM-Gruppe, die mehrsprachige Verbvalenz-Wörterbücher erarbeitet.

(Verantwortlich: Helmut Schumacher)

\section{Graphie und Orthographie}

Im Bereich „Graphie und Orthographie“ wurde als Manuskript abgeschlossen: „Bernhard Rust: Regeln für die deutsche Rechtschreibung 1944“ (Reihe amades IDS, 2 Bd.). Im Band II sind Ergebnisse verwertet, die innerhalb des Projektes „Orthographiedarstellungen im 19. Jahrhundert" gewonnen wurden. Innerhalb dieses Projektes wurde mit der kommentierten Dokumentation „Auseinandersetzungen um die deutsche Orthographie im 19. Jahrhundert" begonnen, und zwar mit den Themen ,Historische Reflexionen", ,s-Schreibung' und ,Groß- und Kleinschreibung' (Reihe Documenta Orthographica Olms Verlag). Zu dem Thema ,Orthographie - Grammatik - Rhetorik“ wurde ein Rohmanuskript erstellt. Das „Archiv zur Geschichte der Orthographie und der Reformbemühungen" wurde um zahlreiche insbesondere amtliche Rechtschreibbücher sowie um den Nachlass des österreichischen Reformers Ernst Pacolt erweitert.

Die Geschäftsstelle der Zwischenstaatlichen Kommission für deutsche Rechtschreibung hatte im Berichtszeitraum mehrere zweitägige Kommissionssitzungen inhaltlich und organisatorisch vorzubereiten. Eine der Sitzungen fand auf Einladung des Ministeriums der Deutschsprachigen Gemeinschaft in Eupen (Belgien), eine andere in Berlin statt. Der Geschäftsführer der Kommission nahm, zum Teil zusammen mit weiteren Kommissionsmitgliedern, auch an Sitzungen des Beirats für deutsche Rechtschreibung teil. Im Hinblick auf den dritten turnusmäßigen Bericht der Kommission wurden im Berichtszeitraum eine Reihe von Untersuchungen zur Umsetzung der neuen Rechtschreibung in den verschiedensten Bereichen betrieben. Fortgesetzt wurde die Zusammenarbeit mit den politisch verantwortlichen Stellen, mit Wörterbuchredaktionen, Nachrichtenagenturen, Medien, Schulbuchau- 
toren und Schulbuchverlagen, mit pädagogischen Psychologen, Rechtschreibdidaktikerinnen und -didaktikern sowie Grammatikern. Weiterhin wurde eine umfangreiche Berater- und Auskunftstätigkeit ausgeübt. Publikationen, Interviews und Podiumsdiskussionen gab es im In- und Ausland.

(Verantwortlich für die Geschäftsstelle: Klaus Heller)

\subsection{Abteilung Lexik}

\section{Abteilungsleiterin:}

Prof. Dr. Ulrike Haß-Zumkehr

Wissenschaftliche Mitarbeiterinnen und Mitarbeiter:

Cyril Belica - Dr. Michael Brodhäcker - Brigitte O. Endres - Sabine Erb (seit 1.7.2001) - Dr. Kristine Fischer-Hupe - PD Dr. Claudia Fraas (bis 31.10.2001) - Daniel Glatz - Prof. Dr. Gisela Harras - Dr. Manfred Hellmann (bis 30.6.2001) - Prof. Dr. Dieter Herberg - Dr. Irmtraud Jüttner (bis 31.3.2001) - Dr. Heidrun Kämper - Meike Lauer (seit 15.5.2001) - Dr. Michael Kinne - Dr. Elisabeth Link - Dr. Anja Lobenstein-Reichmann - Dr. Sonja Müller-Landmann (bis 31.3.2001) - Pantelis Nikitopoulos - Isolde Nortmeyer - Kristel Proost - Dr. Herbert Schmidt - Dr. des. Ulrich Schnörch - Dr. Doris Steffens - Dr. Kathrin Steyer - Dr. Gerhard Strauß - Dr. Elke Tellenbach (bis 30.6.2001) - Oda Vietze - Doris al-Wadi - Christian Weiß (seit 1.7.2001) - Dr. Edeltraud Winkler.

\section{Sekretärinnen:}

Susanne Bergmann (bis 31.10.2001) - Petra Brecht - Joyce-Ann Thompson - Sigrid Ziehr (bis 31.8.2001)

Die Abteilung Lexik erforscht und beschreibt den Wortschatz der deutschen Sprache anhand ausgewählter Elemente (Wörter, Wortteile, Redewendungen) bzw. Bereiche des Wortschatzes (Fremdwortschatz, neuer Wortschatz, Kommunikationsverben). Im Vordergrund des Interesses stehen dabei die Bedeutung und die Verwendungsbedingungen von Wörtern sowie die Veränderungsprozesse, denen der deutsche Wortschatz im Laufe der Zeit unterliegt, und hier vor allem die (zeit-)historischen und die aktuell beobachtbaren Veränderungen. Erforschung und Beschreibung münden in z.T. mehrbändige Wörterbücher und in lexikologische und semantische Konzepte und Methoden. Grundlegend hierfür sind umfangreiche elektronische Korpora schriftlicher (deutschsprachiger) Texte. $\mathrm{Zu}$ den Aufgaben der Abteilung Lexik gehört, diese Korpora kontinuierlich auszubauen und die für eine linguistische Auswertung notwendigen Recherchewerkzeuge zu entwickeln. Um die Forschungsergebnisse aus den verschiedenen Projekten der Abteilung sowohl über einzelne Wörter bzw. Wortschatzelemente als auch über Strukturen innerhalb des Wortschatzes zugänglich zu machen, wird an einem datenbankbasierten und internetfähigen Informationssystem gearbeitet: Wissen über Wörter (WiW), das lexikalisch-lexikologische, korpusba- 
sierte Informationssystem des IDS. Dieses neue Medium ermöglicht die Fortführung bewährter lexikografischer Praxis bei erhöhter Nutzerfreundlichkeit, aber auch die Gewinnung neuer Erkenntnisse über den Wortschatz durch strikte Korpusfundierung, neue Klassifizierungen und vielfach verknüpfbare Suchanfragen.

Im Berichtsjahr wurde ein Projekt der Abteilung inhaltlich abgeschlossen und für die Publikation auf CD-Rom vorbereitet: Das umfangreiche Wörterbuch „Wörter in Texten der Wendezeit - Ein alphabetisches Wörterbuch zum Wende-Korpus des IDS 1989/90", kurz „Wende-Wörterbuch“ (verantwortlich: Manfred W. Hellmann). Es soll in der IDS-Reihe amades - Arbeiten und Materialien zur deutschen Sprache veröffentlicht werden.

\section{Wissen über Wörter (WiW)}

Das Institut für Deutsche Sprache hat Ende 1997 mit dem Aufbau eines „Internet-Wörterbuchs“ begonnen. Im Endausbau wird WiW den Wortschatz der deutschen Sprache (geplant sind 250.000 bis 300.000 Stichwörter) in umfassender Weise dokumentieren, allgemeinverständlich erklären und wissenschaftlich erläutern. Entscheidend ist die Hypertext-Struktur von WiW, die für die Lexikografie eine neue Dimension eröffnet, weil sie den Nutzerinnen und Nutzern, gleich ob Experten oder Sprachinteressierten, eine flexible, auf individuelle Bedürfnisse abgestimmte Informationsauswahl und -tiefe mit entsprechenden Möglichkeiten der Navigation anbietet. Die Vernetzungen zwischen Wortartikeln einerseits und ihren Detailinformationen andererseits dienen nicht nur einer gezielteren Informationsauswahl seitens der Nutzer, sondern lassen auch mehr als bisher üblich über die systematischen Vernetzungen innerhalb des Wortschatzes erkennen. WiW ist als offenes Informationssystem konzipiert, das Schnittstellen zu anderen Projekten und Informationssystemen aufweisen kann und ausbaufähig gehalten wird, denn nur so lässt sich die Dokumentation des deutschen Wortschatzes stets auf aktuellem Stand halten. In die WiW-Datenbank können Ergebnisse aller wortschatzbezogenen Projekte des IDS sowie mittelfristig auch Ergebnisse externer Wortschatzforschungen gebündelt und auch in Beziehung zueinander gesetzt werden. Die Artikelstruktur von WiW unterscheidet sich in wesentlichen Aspekten von der Artikelstruktur gedruckter Wörterbücher, denn hier werden die einzelnen Informationen zu einem Stichwort miteinander und mit den entsprechenden Informationen zu anderen Stichwörtern durch sog. Links vernetzt. So können etwa semantische Netze, Wortfamilien und sachliche Zusammenhänge anschaulich gemacht werden (vergleiche auch: http://www.ids-mannheim.de/wiw/).

Die Daten- bzw. Artikelstruktur des Systems wurde 2001 in einem Kolloquium mit externen Experten anhand von Probeartikeln und Kurzvorträgen zu konzeptionellen Aspekten des Projekts diskutiert, an weiteren Stichwörtern getestet und erneut überarbeitet. Einen großen Stellenwert hatte die Weiterentwicklung teilautomatischer Verfahren zur Erzeugung projektspezifi- 
scher Listen von Partnerwörtern, die wertvolle Hypothesen zu vielen lexikologischen Angaben liefern können. Diese beruhen auf einem besonders zusammengestellten WiW-Korpus, das im Berichtsjahr definiert wurde. Parallel zu diesen Arbeiten wurde ein datenbankbasiertes Software-Paket ausgewählt und es wurde damit begonnen, dieses den Anforderungen des Projekts anzupassen. Priorität hat die Entwicklung eines lexikografischen Arbeitsplatzes, bei dem die Arbeitsteilung von der Materialsammlung über die ,Füllung' bestimmter Informationsfelder bis hin zu Konsistenzprüfung und Endredaktion festgelegt ist. Parallel dazu muss die mehrdimensionale Nutzeroberfläche mit ihrer Verlinkungs- und Navigationsstruktur entworfen werden.

(Verantwortlich: Ulrike Haß-Zumkehr)

\section{Neologismenforschung}

Seit 1997 werden die Neologismen (Neuwörter und Neubedeutungen) der $90 \mathrm{er}$ Jahre des 20 . Jahrhunderts erforscht, soweit sie sich im allgemeinsprachlichen Teil des Wortschatzes der deutschen Standardsprache etabliert haben.

Ziel des Projektes ist die lexikografische Beschreibung und Dokumentation von ca. 800 dieser Neologismen im Rahmen des Internet-Wörterbuchs „Wissen über Wörter (WiW)"(s.o.). Die Wortartikel werden umfassender und detaillierter, als es in einem Printwörterbuch möglich wäre, Informationen zu den herkömmlichen Datentypen sowie zahlreiche Textbelege enthalten. Darüber hinaus werden sie Informationen zu speziellen Datentypen wie Neologismenart, Aufkommen, Buchung in Wörterbüchern, enzyklopädisches Hintergrundwissen vermitteln. Aufgrund der Pilotfunktion, die das Projekt für WiW hat, ist es an der konzeptionellen Entwicklung von WiW beteiligt.

2001 wurden ca. 220 Wortartikel fertiggestellt, die in der DTD-Struktur computergespeichert sowie als Ausdrucke vorliegen. Weitergeführt wurde die Ermittlung von Neologismen.

(Verantwortlich: Dieter Herberg)

\section{Erklärende Synonymik kommunikativer Ausdrücke} des Deutschen (ESKA)

Unter kommunikativen Ausdrücken werden in erster Linie Verben wie auffordern, versprechen, behaupten, mitteilen, loben, erklären usw. verstanden. Für diese Gruppe von Prädikaten ist ein begriffliches Gerüst entwickelt worden, das die semantischen Eigenschaften der Ausdrücke abzuleiten gestattet, so dass die lexikalischen Informationen unter verschiedenen Aspekten repräsentiert sind wie: unter dem Aspekt der Konzeptualisierung der sprachlichen Ausdrücke, unter dem Aspekt ihrer semantischen Feldzugehörigkeit, dem Aspekt ihrer Argumentstruktur in Abhängigkeit von ihrer Konzeptualisierung sowie unter dem Aspekt ihrer jeweiligen lexikalischen Eigenschaften. Dem Benutzer der Datenbank soll so ermöglicht werden, sich die ihn interessierenden Daten(ausschnitte) je nach Bedürfnis zusammenzustellen. 
Im Berichtsjahr 2001 wurden die Gruppen der Direktive (auffordern), Repräsentative (mitteilen) und Kommissive (versprechen) weitgehend abgeschlossen, die Gruppe der Expressive (loben) und Deklarative (erklären) wurde überarbeitet, und die medialen und instrumentalen Prädikate wurden weiter bearbeitet. Es besteht Konsens darin, dass die weitere Arbeit an ESKA bis zur Beendigung noch auf dem alten UNIX-System weitergeführt werden und erst die vollständige Datenbank in eine PC-fähige Version konvertiert werden soll. Die Zusammenarbeit mit der TU Darmstadt (Prof. Dr. Rudolf Wille) wurde fortgesetzt und besonders die Darstellung der Argumentstruktur der Verben im System TOSCANA (Tools for Concept Analysis) feinkörniger gestaltet. Es wurden vier Beiträge verfasst, die in einem Sammelband enthalten sind und sich ausführlich mit der semantischen Komponente von ESKA befassen, vgl. dazu unter DFG-Projekt.

(Verantwortlich: Gisela Harras)

\section{Tendenzen der Lexikalisierung kommunikativer Konzepte (DFG- Projekt)}

In diesem Projekt, das im Mai 1999 begonnen wurde, sollen einmal die speziellen Lexikalisierungseigenschaften von Sprechaktverben und zum andern die Art und Weise ihrer Konzeptualisierung erforscht werden. Es geht also darum, sowohl die Eigenartigkeit der Lexikalisierung dieser Verben als auch ihren Bestand bezüglich eines begrifflichen Gesamtsystems zu untersuchen.

$\mathrm{Zu}$ diesen beiden Problemkreisen sind von den beiden Mitarbeitern umfangreiche Papiere erarbeitet worden, aus denen zwei Aufsätze (von Glatz und Proost) für einen im Berichtsjahr fertig gestellten Sammelband hervorgegangen sind. Zusammen mit den Beiträgen von Harras und Winkler umfasst der Band: Gisela Harras (Hrg.) ,Kommunikationsverben - konzeptuelle Ordnung und semantische Repräsentation" die folgenden Aufsätze: Gisela Harras: „Performativität, Sprechakte und Sprechaktverben“; Daniel Glatz: „Ereignisstruktur von Kommunikationsverben“; Edeltraud Winkler: „Möglichkeit der semantischen Dekomposition von Kommunikationsverben"; Kristel Proost: ,Zum Lexikalisierungsraum kommunikativer Konzepte“; Gisela Harras: ,,Synonymie und Synonymik“; Edeltraud Winkler: „Aufbau und Gliederung einer Synonymik deutscher Sprechaktverben“.

Im Juli 2001 wurde der Fortsetzungsantrag für das Projekt von der Deutschen Forschungsgemeinschaft in vollem Umfang, d. h. für zwei weitere Jahre, genehmigt.

(Verantwortlich: Gisela Harras)

\section{Deutsches Fremdwörterbuch (DFWB)}

Das Deutsche Fremdwörterbuch verzeichnet den deutschen Fremdwortschatz vom 15. Jahrhundert bis zur Gegenwart und stellt mit seiner bedeutungsgeschichtlichen Zielsetzung und umfassenden Quellendokumentation das Standardwerk der historischen Fremdwortlexikographie für Sprachge- 
schichtler, Germanisten, Linguisten, Lexikologen und Historiker aller Bereiche dar.

Im Berichtsjahr wurde weiter an der Fertigstellung von Bd. 5, der die Buchstaben $\mathrm{E}$ und $\mathrm{F}$ (Eau de Cologne - Futurismus) enthalten wird, gearbeitet. Die Arbeiten an den Artikeln zum Buchstaben E wurden - bis auf vereinzelte Nachträge, Kürzungen des Umfangs - zum Beginn des Jahres abgeschlossen (ca. 450-500 Druckseiten). Von den Artikeln der Alphabetstrecke F ist etwa ein Drittel bearbeitet (ca. 250-300 Manuskriptseiten).

(Verantwortlich: Gerhard Strauß)

\section{Frühneuhochdeutsches Wörterbuch (FWB)}

Als „frühneuhochdeutsch“ bezeichnet man das Deutsch, das von der Mitte des 14. Jahrhunderts bis zum 17. Jahrhundert gesprochen und geschrieben wurde. Auf überlieferte Texte bauen wir unser Bild von der Gesellschaft und der Geschichte, indem wir aus den Texten „Wirklichkeiten“ (re)konstruieren. Da es schlechterdings nichts gibt, weder Reales noch Gedachtes, auf das man nicht mittels Wörtern Bezug genommen oder das man nicht gar durch den Wortgebrauch konstituiert hätte, sind sprachliche Zeugnisse vergangener Zeit anderen (oft sehr raren) Überlieferungsträgern weit überlegen und eignen sich besonders als Grundlage historischer (Re)Konstruktion. Die Bedeutung von Wörterbüchern, die die vergangenen Sprachstufen erschließen helfen, ist dabei schwerlich zu überschätzen. Das Frühneuhochdeutsche Wörterbuch, welches eine Lücke in der Reihe der Wörterbücher zu den historischen Sprachstufen des Deutschen schließt, wird daher ein willkommenes Hilfsmittel für all diejenigen werden, die sich mit der Sprache und Kultur der frühen Neuzeit auseinandersetzen, die an der Epoche zwischen der späten Scholastik und der frühen Aufklärung, an der Zeit der Erfindung des Buchdrucks, der Entdeckung Amerikas oder an der Reformation (usw.) interessiert sind.

Im Rahmen der 1993/4 begonnenen Beteiligung des IDS am „Frühneuhochdeutschen Wörterbuch“ (Anderson, Goebel, Reichmann) wurde Bd. 4 (b/p 2. Hälfte) von Joachim Schildt abgeschlossen. Für den Bd. 7 (gn-hz) ist die erste Lieferung von gnaben bis grossprechen fertiggestellt und erschienen (Anja Lobenstein-Reichmann), für den Bd. 12 (f/v) wurden Artikel von $f / v$ bis fechel fertiggestellt (Elisabeth Link).

\section{Historisches Textkorpus}

Weil die regulären IDS-Korpora weitgehend die Sprache der Nachkriegszeit enthalten, wurde von 1992-1998 an Ergänzungen zum 18. und 19. Jahrhundert gearbeitet. Seit 1999 werden Texte des (frühen) 20. Jahrhunderts digitalisiert, um der Erforschung des aktuellen Wortschatzes eine Vergleichsgrundlage zu verschaffen. Dieses Korpus umfasst drei historisch begründete Phasen: die Zeit der Weimarer Republik (1919-1932), die Zeit des Nationalsozialismus (1933-1945) und die frühe Nachkriegszeit (1945-1955). Es 
wurden bis Ende 1999260 Texte aus Politik, Presse, Literatur und Recht mit insgesamt knapp 1 Mio. laufenden Wörtern aufgenommen und zum größten Teil auch schon recherchierfähig aufbereitet. Das Historische Korpus zum 20. Jahrhundert schließt so die Lücke zwischen dem Historischen Korpus zum 18. und 19. Jahrhundert und dem 1999 begonnenen Projekt „Deutsches Referenzkorpus" (siehe Abschnitt "Arbeitsgruppe für Korpustechnologie"), das Texte ab 1956 enthält und über die Gegenwart hinaus fortschreibt. Alle Korpora sind in ähnlicher Weise aufgebaut, so dass vergleichende Recherchen möglich sind.

Der Ausbau des Historischen Korpus konnte im Jahr 2001 aus personellen Gründen nicht wesentlich fortgeführt werden. Es wird in der vorliegenden Form aber bereits intensiv von den diversen lexikografischen Projekten des IDS genutzt.

(Verantwortlich: Ulrike Haß-Zumkehr)

\section{Sprache im Nachkriegsdeutschland (DFG-Projekt)}

Was sagt eine Gesellschaft über sich aus, wenn sie ihre politische Vergangenheit - Adolf Hitler und den Nationalsozialismus - als „Dämon“ „das Böse“ oder die „Macht der Finsternis“ bezeichnet? Was bedeutet es, wenn eine Gesellschaft ihre Gegenwart als "Sintflut", „Endzeit" oder „Chaos“ empfindet? Was heißt es, wenn die Zukunft in Hochwertwörtern wie ,abendländische Kultur“, ,christliche Werte“, „Freiheit und Gerechtigkeit“ versprachlicht wird? Diese und andere Fragen werden in dem seit April 2000 von der DGF geförderten Projekt von einer interdisziplinären Arbeitsgruppe beantwortet, zu der neben zwei Sprachwissenschaftlerinnen auch ein Zeithistoriker gehört. Dem Projekt liegt die Ausgangsüberlegung zugrunde, dass zu keiner anderen Zeit der neueren deutschen Geschichte das Denken in Zeitkategorien so zentral war, wie in der früheren Nachkriegszeit. Daher werden die untersuchten Texte nach den drei Zeitdimensionen geordnet: Aussagen über die vergangene Nazizeit, die Zerstörung und Not der (damaligen) Gegenwart und die künftige Gestaltung der Gesellschaft.

Im Jahr 2000 wurde das Korpus des Projekts festgelegt. Insofern es sich um die Rekonstruktion eines Elitendiskurses handelt, stammen die Quellen aus den Domänen Politik, Theologie/Kirche, Recht, Literatur und Wissenschaft/Philosophie. Eine weitere Gruppe sind Texte aus der Täter-/Opferperspektive. Texte des Korpus werden gelesen, nach einem Klassifikationssystem markiert und digitalisiert/eingescannt. Die Phase der Korpusgewinnung und -strukturierung endete im Dezember 2000. Im Jahr 2001 wurden die klassifizierten Texte (z.T. vor dem Hintergrund einer zeitgeschichtlichen Chronologie) ausgewertet hinsichtlich ihrer Geschichte, ihrer Themen sowie der sprachlichen Repräsentationen der Themen. Grundlage dieser Auswertung ist ihre Reflexion auf der Zeitachse 1945-1955 in Bezug auf die sechs Domänen. Das Ergebnis wird eine monographische Darstellung von Nachkriegsdeutsch als dem Sprachstadium einer Umbruchzeit und die Offenle- 
gung ihrer sprachlichen Ausdrucksform sein, mit deren Abfassung begonnen wurde.

(Leitung: Heidrun Kämper)

Arbeitsgruppe für Korpustechnologie

Die Arbeitsgruppe für Korpustechnologie hat zwei Aufgaben: (1) elektronisches Textmaterial für die Arbeit aller Forschungsprojekte des IDS zu beschaffen, aufzubereiten, zu dokumentieren und als Datenbasis für externe wie interne Wissenschaftler bereitzustellen, die sich mit schriftgebundener Sprache und Wortschatzfragen befassen; (2) die IDS-Korpora geschriebener Sprache zu erschließen und zu analysieren und hierfür Erschließungsmethoden und darauf basierende Analysetechniken zu entwickeln. Elektronische Korpora und die Analysemethoden und -techniken zu deren Erschließung sind als Forschungsinstrumente nicht nur für die linguistische Forschung am IDS zentral, sondern - wie die stets zunehmende Zahl von Online-Nutzern (jährlich ca. 40.000 Online-Nutzungen, davon ca. 30.000 über das WWW, mit ca. 140.000 Suchanfragen) zeigt - auch für die nationale und internationale Germanistik sowie für interdisziplinäre Forschungsinteressen. Das Korpus-Recherche-Instrument COSMAS I steht seit 1997 auswärtigen Nutzern auch über einen WWW-Zugang ohne Registrierungspflicht (http://corpora.ids-mannheim.de/ cosmas) weltweit gebührenfrei zur Verfügung.

Im Jahr 2001 wurden mehrere korpusanalytische Machbarkeits- und Kurzstudien für interne und externe Kollegen z. B. folgender Institutionen durchgeführt: Klinik für Psychosomatik und Psychotherapie, Universität Giessen; Linguistische Datenverarbeitung/Computerlinguistik an der Universität Trier; Lehrstuhl für Psychologie II, Universität Mannheim; Universität Zürich. In Zusammenarbeit mit dem Projekt „Wissen über Wörter“ (WiW) wurde die erste Version einer korpusbasierten Kookkurrenz-Datenbank zu den ca. 1000 frequentesten Lemmata erstellt. Die Arbeitsgruppe hat sich an der Entwicklung des neuen Recherchesystems COSMAS II durch Beratung und durch schrittweise Übergabe von COSMAS-I-Algorithmen zur Integration in COSMAS II beteiligt.

Die IDS-Textkorpora wurden kontinuierlich erweitert um die neuen Jahrgänge der Zeitungskorpora Mannheimer Morgen, Berliner Zeitung, die tageszeitung, um das Korpus „Biografische Literatur“ (Alfred Kerr, Victor Klemperer) und um die Neuakquisitionen im Rahmen des DEREKO-Projekts (s. u.). Zum Korpus „Belletristik des 20. Jahrhunderts“ kamen Werke von Agnes-Marie Grisebach, Stefan Heym, Hans Joachim Schädlich, Klaus Schlesinger, Bernhard Schlink und Christa Wolf hinzu. Das unter dem Recherche-Instrument COSMAS I verfügbare Korpus-Angebot wurde somit auf rund 1130 Millionen Textwörter (das sind etwa 2,8 Millionen Buchseiten) erweitert und ist somit die größte deutschsprachige Korpussammlung weltweit. Im Bereich der bibliographischen Dokumentation wurde das Textmaterial weiterhin in aufwändiger Handarbeit nach dem aktuell gültigen 
Stand des im IDS entwickelten Korpustext-Bibliografiemodells aufgewertet. Über die aktuell verfügbaren Teilkorpora kann man sich auf der Homepage der AG Korpustechnologie (http://www.ids-mannheim.de/kt) eingehender informieren.

(Verantwortlich: Cyril Belica)

\section{Deutsches Referenzkorpus - DEREKO (Drittmittelprojekt)}

Das vom Land Baden-Württemberg finanzierte Kooperationsprojekt „Deutsches Referenzkorpus“ (DEREKO) begann im Mai 1999. Kooperationspartner sind das Institut für Maschinelle Sprachverarbeitung (IMS) der Universität Stuttgart und das Seminar für Sprachwissenschaft (SfS) der Universität Tübingen. Ziel ist, die deutsche Gegenwartssprache (von 1956 bis zunächst 2001) möglichst breit und der Sprachwirklichkeit angemessen abzubilden. Das Projekt ist in die Gesamtvorhaben der Arbeitsgruppe für Korpustechnologie eingebunden und schließt unmittelbar an die Korpusakquisitionsarbeiten des IDS in den vergangenen Jahren an und nutzt die Ressourcen innerhalb des IDS.

In dieser Phase des Projekts lag der Schwerpunkt einerseits auf der Akquisition neuer Texte - hier wurden Korpora im Umfang von mehr als 500 Millionen Wörter zur Test-Nutzung freigegeben - andererseits auf der Fertigstellung eines einheitlichen Textmodells in Form einer DTD (Document Type Definition), die sich an dem internationalen "Corpus Encoding Standard“ orientiert. Die aktuelle DEREKO-Textsammlung enthält mehrere deutsche, Schweizer und österreichischer Tageszeitungen (alle mit mehreren Jahrgängen), ca. 150 belletristische Titel und Sachbücher, eine Sammlung von Texten aus Politik und Recht sowie Computer-Fachtexte. Besonders intensiv wurden im Jahr 2001 die urheberrechtlichen Fragen der künftigen Online-Nutzung dieser Korpora untersucht und juristisch geklärt.

(Verantwortlich: Cyril Belica und Ulrike Haß-Zumkehr)

\subsection{Abteilung Pragmatik}

\section{Abteilungsleiter:}

Prof. Dr. Werner Kallmeyer

\section{Wissenschaftliche Mitarbeiterinnen und Mitarbeiter:}

Dr. Jannis Androutsopoulos - Dr. Karl-Heinz Bausch - PD Dr. Nina Berend - Ibrahim Cindark - Sylvia Dickgießer - Dr. Mechthild Elstermann - Prof. Dr. Reinhard Fiehler - Daniela Heidtmann (ab 1.3.2001) - PD Dr. Inken Keim-Zingelmann - Ralph Knöbl - Prof. Dr. Katharina Meng - Ulrich Reitemeier - Dr. Rudolf Schmidt - Dr. Reinhold Schmitt - Dr. Wilfried Schütte PD Dr. Thomas Spranz-Fogasy - Dr. Peter Wagener (beurlaubt bis 30.6.2001).

Toningenieur: Wolfgang Rathke

Sekretärinnen: Doris Richter - Ulrike Willem 
Forschungsgegenstände der Abteilung sind sprachliches Handeln und sprachliche Variabilität, d.h. die Ausprägung und Entwicklung von Sprachunterschieden innerhalb des Deutschen. Das Aufgabenfeld umfasst im Einzelnen:

- die Formen des sprachlichen Handelns und ihre Einbettung in die gesellschaftliche Kommunikationsstruktur;

- die Beziehung zwischen der Sprachverwendung und ihren medialen Bedingungen;

- die Sprachvariation im Deutschen und Sprachkontakterscheinungen in Mehrsprachigkeitssituationen unter Beteiligung des Deutschen.

Im Berichtszeitraum konzentrierten sich die Arbeiten auf die folgenden Projekte:

\section{Sprachliche Integration von Aussiedlern}

Das Projekt beschäftigte sich mit der Frage, wie sich Aussiedler aus der GUS und Polen in die Sprach- und Kommunikationsgemeinschaft integrieren, die sie in Deutschland vorfinden. Die Abschlusspublikationen sind im Jahr 2001 erschienen bzw. werden für die Publikation vorbereitet. Die Ergebnisse des Projekts wurden in einem internationalen Kolloquium „Sprachliche Integration von Aussiedlern in den 90er Jahren - Forschungsbilanz und Forschungsperspektiven“ (IDS, 5.-7.4.01) vorgestellt.

\section{Eigenschaften gesprochener Sprache}

Die Arbeit zielt darauf ab, die Variabilität gesprochener Sprache herauszuarbeiten und mündliche Kommunikation - im Vergleich mit Schriftlichkeit und technisierter Kommunikation - durch die Diskussion ihrer verschiedenartigen Grundbedingungen als eigenständiges Verständigungssystem zu verdeutlichen. Eine Abschlusspublikation wird vorbereitet.

\section{Gesprächsrhetorik}

Eine umfangreiche systematische Darstellung zur „Gesprächsrhetorik“, die vor allem verbale Muster und Verfahren in Problem- und Konfliktgesprächen behandelt, wird zum Druck vorbereitet. Außerdem werden die Projektergebnisse und Beispielanalysen aus der Projektarbeit in das Gesprächsanalytische Informationssystem GAIS aufgenommen.

\section{Gesprächsanalytisches Informationssystem (GAIS)}

Entwickelt wird ein multimediales Informationssystem für die gesprächsanalytische Arbeit von Wissenschaftlern und Studenten. GAIS bietet Informationen und Arbeitsmöglichkeiten zur Gesprächsanalyse allgemein und speziell zu Ergebnissen und Verfahren der Gesprächsrhetorik. Das System umfasst ein Lexikon zur Gesprächsanalyse, Beispielanalysen, Korpora für weitere Analysen, Aufgabenstellungen und Lösungen für das Selbststudium 
sowie eine Literaturdatenbank. Ein wichtiger Teil des Systems sind außerdem Programme für die Korpusarbeit und die Aufbereitung von Daten gesprochener Sprache von der Transkription (DIDA) über die Synchronisation von Transkriptionstext und Ton (ALIGNER) bis zur Recherche in den Transkriptionsskorpora (COSMAS II). Die inhaltliche Strukturierung ist im ersten Schritt auf einen für den Hochschulbereich konzipierten Kurs „Gesprächsrhetorik: Beispiel Fernsehgespräche“ ausgerichtet und wird dann fortlaufend auf weitere Themen ausgeweitet.

Kommunikative soziale Stilistik

Zentrales Vorhaben der Abteilung ist das Projekt „Kommunikative soziale Stilistik“. Ziel ist die Erarbeitung einer Soziostilistik der Kommunikation in Deutschland. Im Zentrum steht der Zusammenhang zwischen der Ausprägung von kommunikativen Stilen, die als Ausdruck der sozialen Identität von gesellschaftlichen Gruppen und Individuen fungieren, und sozialen Prozessen der Integration, Differenzierung, Distanzierung und Ausgrenzung. Ergebnis ist eine grundlegende Darstellung von allgemeinen Stilbildungsprinzipien und Prozessmustern der sprachlich-sozialen Entwicklung, die an einer aspektreichen Auswahl von Kommunikationsvorgängen in unterschiedlichen Domänen der Gesellschaft demonstriert werden. Den Orientierungsrahmen für die einzelnen empirischen Untersuchungen bilden Dimensionen der gesellschaftlichen Gliederung, die in soziologischen und kulturwissenschaftlichen Arbeiten als Grundeigenschaften gesellschaftlicher Realität angesehen werden und deren Ausprägungen für die Bestimmung moderner Gesellschaften herangezogen werden:

- Vertikale Gliederung („Oben und Unten“). Durchgeführt wird ein Teilprojekt „Das kommunikative Handeln von Führungskräften“.

- Arbeit und Freizeit. Es laufen gegenwärtig zwei Teilvorhaben: „Kommunikation in Arbeitsgruppen“ und „Internetkommunikation als Arbeitsinstrument".

- Migration („Ansässige und Zuwanderer“). Hier läuft ein Teilprojekt „Sprachvariation Deutsch-Türkisch und kommunikative soziale Stile von Migranten". Ein weiteres Projekt zur kommunikativen Stilistik in der Welt russlanddeutscher Aussiedler wird vorbereitet.

- Lebensalter und Generationswechsel (,Jung und Alt"). Gegenwärtig läuft ein Teilprojekt „Stilbildung und Variationspraxis in der medialen und unmittelbaren Kommunikation von jugendkulturellen Sozialwelten“. Vorbereitet wird ein Teilvorhaben zur Alterskommunikation.

- Öffentlichkeit und Privatheit. Dieser Arbeitsbereich wurde vorerst zurückgestellt.

Zwei der genannten Teilvorhaben (,Sprachvariation Deutsch-Türkisch und kommunikative soziale Stile von Migranten“ sowie „Stilbildung und Variationspraxis in der medialen und unmittelbaren Kommunikation von jugend- 
kulturellen Sozialwelten") sind zugleich Teilprojekte im Rahmen der von der DFG geförderten Mannheimer Forschergruppe „Sprachvariation als kommunikative Praxis", die ihre Arbeit am 1.3.2000 aufgenommen hat.

Lautliche Variation des Deutschen -

Standardsprache und regionale Umgangssprachen

Das Projekt wird gegenwärtig vorbereitet. Ziel ist eine Datenbank von Aussprachevarianten deutscher Wörter in der Standardsprache und in den regionalen Umgangssprachen. Die Datenbank kann zur Herstellung eines Aussprachewörterbuches für spezifische Adressatenkreise (u.a. Deutsch als Fremdsprache) genutzt werden. Längerfristiges Ziel ist die Klärung des Zusammenhanges zwischen Sprachvariation und Prozessen des Sprachwandels.

Korpusausbau und Entwicklung von Korpustechnologie

Zunehmende Bedeutung bekommt der Bereich Korpusausbau und Entwicklung von Korpustechnologie für Daten gesprochener Sprache. Das korpustechnologische Ziel ist die Einrichtung eines vernetzten Gesamtsystems (Eingabe, Speicherung und Zugriff) für Korpora gesprochener Sprache. Die wichtigsten Komponenten eines solchen Systems sind ein Transkriptionseditor, ein System für Text-Ton-Alignment, eine Datenbank für Transkripte und Zeitmarken, eine Datenbank für den Zugriff auf sämtliche dokumentarische Daten, ein Recherchesystem und ein Ausgabesystem für Transkripte mit Zugriff auf die Tondaten. Die Arbeiten an den folgenden Komponenten des Gesamtsystems wurden im Berichtsjahr weitergeführt. Abgeschlossen wurden zwei drittelfinanzierte Projekte:

- Text-Ton-Alignment (ein Kooperationsprojekt mit dem Institut für maschinelle Sprachverarbeitung der Universität Stuttgart, Abschluss Ende Juni 2001),

- Datenbank Gesprochenes Deutsch für die computerunterstützte Erfassung und Erschließung der Tonaufnahmen des Deutschen Spracharchivs, der Verwaltungsinformationen und der Transkripte; ein drittmittelfinanziertes Projekt für die Programmierung des Systems wurde im Juli 2001 abgeschlossen. Die Datenbank wird sukzessive mit den vorhandenen digitialisierten und transkribierten Aufnahmen zum gesprochenen Deutsch gefüllt.

\section{Deutsches Spracharchiv (DSAv)}

Aufgabe des Deutschen Spracharchivs (DSAv) ist die Archivierung und Dokumentation von Daten gesprochener Sprache. Hier sind auch die Aufgaben für den internen und externen Service angesiedelt. Die zentrale Dokumentation aller in der Abteilung verwalteten Korpora gesprochener Sprache wurde fortgesetzt, ebenso die Inventur der vorhandenen Begleitmaterialien zum wichtigsten Dialektkorpus (Zwirner-Korpus). 
2.4 Arbeitsstelle Zentrale DV-Dienste (ZDV)

\section{Leiter:}

Eric Seubert

Wissenschaftliche Mitarbeiter:

Irfan Bilgili (ab 17.4.2001) - Franck Bodmer

Kaufmännisch-technischer Mitarbeiter:

Bernd Rolzhäuser

\section{Technische Mitarbeiterinnen und Mitarbeiter:}

Siegmund Gruschka - Matthias Hördt - Rainer Krauß - Ingrid Schellhammer

\section{Sekretärin:}

Irmgard Schlösser (bis 31.10.2001) - Susanne Bergmann (ab 1.11.2001)

Die ZDV definiert sich durch ihren Namen als die Arbeitsstelle, in der die zentralen Datenverarbeitungsdienste des IDS abgewickelt werden.

Sie hat die Aufgabe, die wissenschaftlichen Arbeiten der Forschungsabteilungen durch Bereitstellung und Pflege der erforderlichen Hard- und Software zu unterstützen. Hinzu kommen wissenschaftliche und nichtwissenschaftliche Programmierarbeiten in Zusammenarbeit mit den anderen Abteilungen und Arbeitsstellen.

Die Arbeiten der ZDV umfassen zur Zeit

- Sicherung des Betriebs der Rechner im IDS,

- Betreuung des lokalen Netzwerks und der zentralen Internet-Dienste des IDS,

- Erstellung von projektspezifischen Programmen, Skripten und sonstigen Bausteinen,

- Betreuung zentraler Software (darunter die Datenbanksysteme ORACLE und TAMINO) und Entwicklung zugehöriger Anwendungen,

- Entwicklung und Betreuung des Korpus-Recherchesystems COSMAS II sowie

- Mitarbeit bei Konzeption, Erstellung und Aktualisierung des Informationsangebots des IDS im WWW.

Technische und administrative Arbeiten an PCs

Die durch Abgänge einer Reihe von Mitarbeitern oder Neuanschaffung freigewordenen Rechner wurden nach vorliegenden Anträgen entsprechend aufgerüstet, konfiguriert und anschließend neuen Benutzern zugestellt.

Im vergangenen Jahr wurden so 30 neue PCs angeschafft, installiert und konfiguriert. Von den freigewordenen PCs wurden wiederum 50, wenn notwendig aufgerüstet, umkonfiguriert und ihren neuen Benutzern zugestellt. Die Anzahl der Diagnosen und nachfolgenden Reparaturen an Rechnerhard- 
ware, die in Eigenverantwortung von Mitarbeitern der Arbeitsstelle durchgeführt wurden, belief sich auf ca. 50 .

Die Datenbank zur besseren Verwaltung der PC-Hardware und -Software im Haus wurde strukturell weiter ausgebaut und mit Daten angereichert. Durch die Datenbank kann die bestehende Inventarisierung von DV-Materialien korrigiert, erweitert und aktualisiert werden.

Netzwerkanbindung und Netzwerkausbau

Die Netzwerkanbindung an das Weitverkehrsnetz von bisher $2 * 64 \mathrm{kBit} / \mathrm{sec}$ wurde auf vorläufig $2 \mathrm{Mbit} / \mathrm{sec}$ verbessert. Als Übertragungsmedium wird statt einer gemieteten ISDN-/Glasfaserleitung eine Richtfunkstrecke, die vom IDS zum Rechenzentrum der Uni Mannheim verläuft, benutzt. Bis die maximale Übertragungsrate von $10 \mathrm{Mbit} / \mathrm{sec}$ voll einsetzbar ist, wird mit der etwas geringeren Rate von $2 \mathrm{Mbit} / \mathrm{sec}$ gefahren.

Im dritten Quartal des Jahres wurde die Erweiterung des lokalen Netzwerks fortgesetzt. Die zunächst als drei sukzessive Schritte konzipierten Arbeiten konnten im Berichtsjahr in einem Zug ausgeführt werden. Diese umfassten neben der Installation der Etagen-Switches (zwei pro Stockwerk) die entsprechende Neuverkabelung aller Räume in den jeweiligen Etagen. Der Datendurchsatz auf der Backbone konnte damit von bisher $10 \mathrm{Mbit} / \mathrm{sec}$ (Ethernet 10b2) um das $200 \mathrm{fache}$ auf $2 \times 1 \mathrm{Gbit} / \mathrm{sec}$ (Glasfaser) gesteigert werden. Zur qualitativen Verbesserung in Form einer erhöhten Datenbandbreite sowie einer modernen Netzwerktechnologie und -topologie gesellt sich eine quantitative: die Anzahl der vorhandenen, aber noch nicht genutzten Netzwerkanschlüsse lässt für die nächsten Jahre Spielraum.

Mit Ausnahme der Bibliothek kann bereits jetzt das ganze IDS am Glasfasernetz partizipieren.

Das veraltete 10 -Mbit-Netzwerk samt alter Zimmerverkabelung bleibt für Notfälle bestehen.

In Ergänzung zur PC-Datenbank wurde eine Netzwerk-Datenbank eingerichtet, in der die neue Verkabelung datentechnisch aufbereitet ist.

\section{Zentrale Hardware und Software}

Nachdem der IDS-Server bereits in den vorangegangenen Jahren nach und nach als zentraler Server des IDS abgelöst wurde, musste er im Lauf des Jahres endgültig außer Betrieb genommen werden. Die bis zuletzt noch verbliebenen Dienste - Terminalserver für die X-Terminals der Abteilung „Pragmatik“ und Backup-Server für verschiedene Netzwerk-Dienste - wurden auf einen herkömmlichen, leicht veralteten PC unter Linux migriert. Die im Vorfeld gesicherten Daten wurden den Benutzern auf dem „neuen“ Rechner wieder zur Verfügung gestellt.

Ebenfalls abgeschaltet wurde der SINIX-Mehrplatzrechner MX300B (nach dem MX300A im vorangegangenen Jahr). Sein kompletter Datenbe- 
stand wurde gesichert und archiviert, die Benutzeranschlüsse wurden auf einen der verbleibenden MX300-Rechner umgehängt.

Der WWW-Server wurde wegen ständig steigender Zugriffe und der Einbindung anspruchsvollerer WWW-Applikationen auf einen leistungsstärkeren Rechner migriert. Der zuvor benutzte Rechner fungiert als BackupRechner unverändert weiter.

Aufgrund mangelnder Auslastung wurde für den Rechner für Statistikanwendungen (Mehrprozessor-Rechner mit zwei PentiumPro-Prozessoren) ein neues Anwendungsprofil erstellt. Nach einer deutlichen Erweiterung des Festplattenspeichers (durch Anschaffung eines externen Plattenarrays, das bis zu sieben Festplatten mit einer gesamten Kapazität von $250 \mathrm{~GB}$ aufnehmen kann) wird der Rechner nun für Multimediaanwendungen und als Speicher für Multimediadaten verwendet.

Statt des vorhandenen Betriebssystems DG/UX, das mittlerweile eine Insellösung im IDS darstellt, wurde eine aktuelle Version von LINUX eingespielt.

Anfang des Jahres wurde die aktuelle Version von ORACLE (8.1.7) auf dem zentralen Applikationsserver installiert. Das IDS verfügt damit wieder über ein zentrales und modernes Datenbanksystem, das über eine Reihe von Anwendungen allen Benutzern im Haus und weltweit über das WWW zur Verfügung steht.

Auf dem gleichen Rechner wurde auch das XML-basierte Datenbanksystem TAMINO installiert und in Betrieb genommen. Durch die Verwaltung von TAMINO und seiner Anwendungen unterstützt die ZDV nicht nur technisch, sondern in DV-Hinsicht auch konzeptionell die Forschungsarbeiten am Projekt „Wissen für Wörter" der Abteilung „Lexik“.

Zur optimalen Nutzung der Ressourcen von ORACLE und TAMINO wurden und werden die zuständigen Mitarbeiter und Mitarbeiterinnen umfassend geschult.

\section{Zentrale Datenbanksysteme und -anwendungen}

Die technische Betreuung des grammatischen Informationssystems GRAMMIS der Abteilung „Grammatik“ umfasste neben der Weiterentwicklung der GRAMMIS-Anwendungen

- die Datensicherung und den Datentransfer für den GRAMMIS-Server und den Datenbankserver,

- die Betreuung des GRAMMIS-Servers und der GRAMMIS-Anwendung auf dem Datenbankserver,

- die Benutzerbetreuung für GRAMMIS.

Für das Projekt ProGr@mm der Abteilung „Grammatik“ wurden die allgemeine technische Betreuung sowie die Implementierungsarbeiten übernommen. Die erste öffentliche Präsentation im Rahmen eines Seminars an der Universität Mannheim verlief nach einem mehrwöchigen Testeinsatz erfolg- 
reich. Die Arbeiten am Projekt sind noch nicht abgeschlossen, weitere Anforderungen werden sich im Einsatz entwickeln. Die Anwendung basiert (analog zu GRAMMIS) auf dem PL/SQL-Toolkit von ORACLE.

Für das DFG-Projekt „Zeitreflexion im ersten Nachkriegsjahrzehnt“ der Abteilung „Lexik“ wurde das im vorherigen Jahr entwickelte Dateiverwaltungssystem unter ORACLE weiter ausgebaut und verwaltet.

Im Rahmen der Arbeiten zum Deutschen Fremdwörterbuch der Abteilung "Lexik“ wurde mit der Implementierung von Programmen zur automatischen Erzeugung eines Basis-Stichwortregisters aus den vorliegenden $\mathrm{Be}-$ legartikeln der Buchstaben A-E heraus begonnen. Die Bereitstellung einer ORACLE-Datenbank mit zugehörigen Masken zur Recherche in den Registerdaten wird im kommenden Jahr vorgenommen.

Die Projektgruppe des IDS-Projekts „Wissen über Wörter“ der Abteilung „Lexik“ wurde über geeignete technische Lösungen beraten. In diesem $\mathrm{Zu}$ sammenhang wurden Funktionen ausgestestet, die u. a. ORACLE und TAMINO für die Verwaltung von XML-Dokumenten und ihrer Darstellung im WWW bieten. Die effizientere Umsetzung der Vorstellungen der Projektgruppe gab den Ausschlag für den Einsatz von TAMINO. Die dazugehörigen Arbeiten wurden bereits in Angriff genommen.

Das Katalogisierungssystem BISLOK der Bibliothek wurde ebenso wie die Datenbank ESKA der Abteilung „Lexik“ in gewohnter Weise technisch betreut.

\section{Korpusrecherchesystem COSMAS II}

Die ursprünglich für das Berichtsjahr geplante institutsweite Einführung von COSMAS II und die damit verbundene Ablösung von COSMAS I mussten auf Anfang des Jahres 2002 verschoben werden, da sowohl die Ausführungsgeschwindigkeit einiger Funktionen als auch der Umfang einzelner Funktionalitäten von COSMAS II (vor allem im Vergleich mit COSMAS I) noch nicht den Anforderungen der Benutzer im IDS entsprach. Im Januar 2002 wird eine gründlich überarbeitete und verbesserte Version von COSMAS II herausgegeben.

Im laufenden Jahr wurden neben zahlreichen kleineren Verbesserungen die Funktionalitäten für die Lemmatisierung und die Kollokationsanalyse vervollständigt. Das Hauptaugenmerk lag auf der Reduzierung der Ausführungszeiten für Datenbankaufbau, Suche, Sortierung, Kontext- und Frequenzermittlung. So konnten durch Implementierung einer alternativen Indexierungsmethode die Geschwindigkeiten für die Suche deutlich erhöht werden. Eine drastische Reduzierung des Archivumfangs ließ die Ausführungszeiten für die Sortierung und Kontextermittlung ebenso deutlich sinken.

Weitere Performanzsteigungen lassen sich möglicherweise durch parallelen (statt bisher sequenziellen) Zugriff auf die Textkorpora erreichen - unter der Voraussetzung einer entsprechenden Anpassung der Hardware. 
An der COSMAS-II-basierenden Anwendung zur Benutzung der Datenbank Gesprochenes Deutsch des „Deutschen Spracharchivs“ (Abteilung „Pragmatik“) wurden mehrere Korrekturen und kleinere Erweiterungen vorgenommen.

\section{Korpusbasierte Arbeiten}

Für die Arbeitsgruppe „Korpustechnologie“(Abteilung „Lexik“) wurden das ganze Jahr über routinemäßig die aktuellen Ausgaben der Tageszeitungen „Mannheimer Morgen“, „Berliner Zeitung“ und „taz“ über Konvertierungsroutinen als Korpora für COSMAS (I + II) verfügbar gemacht. Daneben konnten Werke von Stefan Heym, Martin Walser, Victor Klemperer u. a. nach entsprechender Konvertierung den COSMAS-Korpora hinzugefügt werden.

In Zusammenarbeit mit der Projektgruppe DeReKo (Arbeitsgruppe „Korpustechnologie“ der Abteilung „Lexik“) wurden die Arbeiten zur Konvertierung der in COSMAS eingebundenen Korpora aus den im IDS entwickelten und bisher benutzten „Mannheimer Konventionen“ in den SGML-basierenden Standard CES weiter voran getrieben. Nachdem von der Projektgruppe eine Umsetzungstabelle für Primärtext festgelegt wurde, konnten mit Hilfe des eigenentwickelten Korpus-Transformationscompilers TraDuCES alle von der Projektgruppe akquirierten Korpora konvertiert und nach erfolgreichem Abgleich mit der zugehörigen DTD den Kooperationspartnern von DeReKo zum Austesten zur Verfügung gestellt werden.

Eine Umsetzungstabelle für die bibliografischen Daten (BOT-Header) konnte von der Projektgruppe dagegen noch nicht vorgelegt werden. Eine Konvertierung dieser Daten ist daher nur sehr rudimentär möglich.

Derzeit laufen unabhängig von DeReKo die Arbeiten an der Konvertierung der IDS-eigenen Korpora.

\section{WWW-Service des IDS}

Im Bereich WWW-Service des IDS wurde zur konsistenten und aktuellen Haltung von (öffentlichen) Personalinformationen ein Prozedurpaket erstellt, das - völlig automatisiert - diese Informationen für verschiedene Anwendungsgebiete zur Verfügung stellt. Darunter fallen zum einen für die Ausgabe im WWW

1. die automatische Erzeugung zentraler Übersichtsseiten über alle Mitarbeiter/Hilfskräfte des IDS,

2. die automatische Erzeugung dezentraler Übersichtsseiten über die Mitarbeiter/Hilfskräfte der jeweiligen Abteilungen/Arbeitsstellen und

3. die automatische Authentifizierung des alphabetischen Katalogs der IDSPublikationen.

Zum anderen können aus den Personalinformationen nebenbei - ebenfalls automatisch - diverse interne Mailinglisten erzeugt und ständig aktuell gehaiten werden. Zuletzt wird auch die DV-Benutzerverwaltung (Benutzerken- 
nungen auf zentralen Rechnern, Mail-Adressen und zugehörige Mailboxen) erheblich erleichtert.

Die notwendigen Daten zu Ein- und Austritt sowie Beschäftigungsart und Einsatzort aller Mitarbeiter und Hilfskräfte werden von der Arbeitsstelle „Verwaltung" beigesteuert.

$\mathrm{Zu}$ den Aufsätzen der Zeitschrift Deutsche Sprache werden in Zusammenarbeit mit der Arbeitsstelle „Öffentlichkeitsarbeit und Dokumentation" mittlerweile auch rückwirkend die jeweiligen Abstracts im WWW verfügbar gemacht.

2.5 Zentrale Arbeitsstelle Öffentlichkeitsarbeit und Dokumentation

Leiterin:

Dr. Annette Trabold

Wissenschaftliche Mitarbeiterinnen und Mitarbeiter:

Franz Josef Berens - Dr. Sabine Karl - Monika Kolvenbach

EDV-Mitarbeiter:

Claus Hoffmann

Erstellung von Druckvorlagen:

Cornelia Häusermann - Joachim Hohwieler - Norbert Volz

Bibliothek:

Lucia Berst - Evgeneia Friedebach (bis 1.8.2001) - Birgit Günther - Eva Teubert (Leitung)

\section{Sekretariat/Sachbearbeitung:}

Barbara Stolz

In der Arbeitsstelle sind die Bereiche

- Öffentlichkeitsarbeit und Presse

- Publikationswesen

- Dokumentation

- Bibliothek

organisatorisch zusammengefasst.

$\mathrm{Zu}$ den laufenden Aufgaben der Arbeitsstelle zählen: Pflege von Pressekontakten, Redaktion der Zeitschrift „Sprachreport", Redaktion der Zeitschrift „Deutsche Sprache“, Gästebetreuung, Zusammenstellen von Besucherprogrammen, Betreuung des Vereins der Freunde des IDS, Bearbeitung von Praktikumsanfragen, Tagungsorganisation, Druckvorlagenerstellung für die IDS-Publikationen (siehe Kapitel 10), Erstellung von Präsentationspostern für Projekte und Abteilungen, Zentrale Adressverwaltung, Vertrieb der Eigenverlagspublikationen und die Bearbeitung bzw. themenspezifische Verteilung von Anfragen. Die Anfragen per E-Mail wachsen durch die allgemein steigende Internet-Nutzung weiterhin an. 
Neben diesen laufenden Aufgaben war der Schwerpunkt der Öffentlichkeitsarbeit 2001 die Organisation und Durchführung der Jahrestagung zum Thema „Sprache und Recht".

Erneut fanden in den Räumen des IDS zwei Ausstellungen statt, die erste trug den Titel „ÜberBLAU“ und zeigte Arbeiten von Christiane Gall-Hieber, Mannheim. Die zweite Ausstellung präsentierte unter dem Titel,thema_tisch“ zahlreiche in Bilder umgesetzte Sprachspiele. Auch das Thema ,Anglizismen in der deutschen Sprache" sorgte für Medieninteresse und Schlagzeilen. Das IDS wurde mehrfach um Stellungnahmen und Kommentare gebeten.

Die „Dokumentation zur Germanistischen Sprachwissenschaft: Sprachwissenschaftliche Forschungsvorhaben“ ist als abfragbare Datenbank im WWW veröffentlicht. (http://www.ids-mannheim. de/oea/forsch/). Diese Datenbank wird stets aktualisiert. Rund 890 Projekte sind zum Jahresende verzeichnet; der Schlagwortkatalog wird regelmäßig terminologisch kontrolliert.

Das Informationsangebot im Internet über das IDS ist im Vergleich zum Vorjahr um weitere 600 Seiten auf nun rund 2200 Seiten angewachsen. Monatlich können seit der zweiten Jahreshälfte 2001 im Durchschnitt 250.000 Zugriffe aus dem In- und Ausland auf IDS-Seiten gezählt werden. Das sind rund 90.000 mehr als in der zweiten Jahreshälfte 2000. In Zusammenarbeit mit der Arbeitsstelle Zentrale DV-Dienste wird das Informationsangebot des IDS im WWW weiter ausgebaut - so werden etwa Abstracts auch zurückliegender Ausgaben zu Aufsätzen der Zeitschrift „Deutsche Sprache“ erstellt - und stetig aktualisiert. Aktuelle Informationen über das IDS können unter $\mathrm{http}: / / \mathrm{www}$.ids-mannheim.de abgerufen werden.

In der Bibliothek werden mit dem Katalogisierungssystem BISLOK sämtliche Neuerwerbungen und - soweit möglich - Altbestände erfasst. Sie sind über Stich- und Schlagwortsuche auch sachlich erschließbar, seit der Kata$\log$ der Bibliothek online nutzbar ist.

Ausländische Gastwissenschaftler und Nutzer aus den umliegenden Hochschulen werden durch die Bibliotheksangestellten beraten, wobei nicht nur Beratungen in der Bibliothek selbst, sondern vermehrt solche per Post, Telefon und E-Mail zu verzeichnen sind.

Die von der Bibliothek herausgegebenen Neuerwerbungslisten (Erscheinungsweise 3-4 Mal jährlich) dienen der in- und ausländischen Hochschulgermanistik als wichtige Informationsquelle. Sie werden auf Anforderung verschickt und sind darüber hinaus monatlich im Internet aktualisiert abrufbar über http://www.ids-mannheim.de/oea/neueingang.html.

\subsection{Verwaltung und Vorstandssekretariat}

\section{Verwaltungsleiter:}

Harald Forschner

\section{Verwaltungsangestellte:}

Jean Christoph Clade - Gerhard Köck - Hildegard Magis (bis 31.7.2001) Gerd Piroth - Heike Ripke - Irmgard Schlösser - Hermann Schmitt 
Telefonzentrale/Poststelle:

Sibylle Heiss-Foster - Hannelore Wittmann

Hausmeister: Uwe Zipf

Direktionsbüro: Cornelia Pfützer-König

2.7 Hilfskräfte am IDS (Stand November 2001)

Abteilung Grammatik

Tamara Altmann; Hagen Augustin; Bouchra Boukhriss Clemente; Christian Drexler; Luis Escarate; Ilona Anna Ewald; Mareike Hafner; Natascha Korol; Beata Kouchnir; Bela Nemoda; Boris Nicklas; Olga Posovitnyuk; Julia Reichert; Jutta Reinisch; Heiko Schoberwalter; AnnaVolodina; Wiebke Wagner

\section{Abteilung Lexik}

Juliane Borm; Saskia Dams; Mirko Ganz; Tanja Klankert; Ilona Link; Ute Paulokat; Stefaniya Ptashnyk; Ulla Radtke; Roland Reibeling; Jarek Schlauch; Greta Stanaityte; Kristina Türschmann; Nilgün Yavuz

\section{Abteilung Öffentlichkeitsarbeit, Dokumentation, Bibliothek}

Thirza-Kathryn Albert; Dagmar Klitzsch; Sabine Mantel; Alexander Michailidis; Monika Schäfer; Christina Schepers; Liping Yan

\section{Abteilung Pragmatik}

Asel Baiborieva; Necmiye Ceylan; Elisabeth-Christine Gamer; Mirko Ganz; Fabian Hörack; Richard Jäger; Julia Kommert; Daniel Kraft; Nina Nikolic; Azim Yasar Özdemir; Björn Peter; Andrea Scheinert; Ersoy Sentürk; Emran Sirim

\section{Tagungen, Kolloquien und Vorträge externer Wissenschaftler am IDS}

\subsection{Sprache - Recht - Öffentlichkeit}

\section{Jahrestagung des IDS vom 13. - 15.3.2001}

In seiner Eröffnungsansprache umriss der Direktor des IDS, Gerhard Stickel, den Gegenstand der Tagung. Wer sich mit dem Thema ,Sprache und Recht" beschäftigt, befasst sich mit dem Sprachgebrauch im Rechtssystem, in der Verwaltung und in der Politik - unter historischen und pragmatischen Fragestellungen. Die Sprache des Rechts ist dem Alltag sehr nah, sie enthält wenig Fremdwörter - und doch sind Rechtstexte oft unverständlich für $\mathrm{Au}$ ßenstehende. Die Frage nach der Verständlichkeit spielt daher schon immer eine zentrale Rolle bei der Beschäftigung mit Sprache und Recht. Auch der Kulturbürgermeister der Stadt Mannheim, Peter Kurz, hob in seinem Gruß- 
wort das Problem der Verständlichkeit hervor: Recht ist geronnene Politik, und wer das Recht nicht mehr versteht, der versteht auch die Politik nicht mehr. Bloße Vereinfachung beseitigt dieses Problem jedoch nicht, vor allem dann nicht, wenn es durch mangelnde Kenntnisse auf der Empfängerseite bedingt ist. Ein solcher Sprachverlust ist eigentlich ein Demokratieverlust, den Kurz vor allem durch vereinfachende mediale Inszenierungen befürchtet.

Das Spannungsfeld von Sprache, Recht und Öffentlichkeit leuchtete der Sprachwissenschaftler Georg Stötzel anhand der Beziehung von gesellschaftlichem Wandel zu Rechtswandel aus. Öffentlichkeit und Recht sind Diskurssysteme, deren Verhältnis dann brisant wird, wenn sich gesellschaftliche Wertungen derart verändern, dass davon auch rechtliche Regelungen betroffen sind. Werte sind sprachvermittelt, und wenn sich Werte wandeln, wandelt sich auch die Sprache - die Gerichte müssen dem in ihren Urteilen Rechnung tragen. Allgemein wird auch erwartet, dass das Rechtssystem sich dem gesellschaftlichen Wertewandel anpasst. Die Aufhebung von Straftatbeständen wie „Ehebruch“ oder „Unzucht zwischen Männern“, die Einführung des Straftatbestandes „Vergewaltigung in der Ehe“ sind Beispiele hierfür.

Sprachveränderungen zeigen daher an, dass sich die Interpretation der Wirklichkeit verändert hat. Hierbei ist die Justiz oft zeitnäher am Bedeutungswandel des Wortschatzes als die Redaktionen von Wörterbüchern. Polizisten bezeichneten sich schon 1980 selbst als „Bulle“ und daraus schlossen Gerichte, dass die Verwendung von „Bulle“ allein noch keine Beleidigung ist - im Wörterbuch hingegen wurde noch 1999 „Bulle“ ausschließlich als negative Fremdbezeichnung für „Polizist" geführt.

Auch „Zeitpunkt des Todes“ ist ein Beispiel für gesellschaftliche Umwertungen - medizinisch-technischer Wandel macht juristische Umdefinitionen nötig. Mit dem Einsatz von Herz-Kreislauf-Maschinen verschob sich der Zeitpunkt des Todes vom Herz- zum Hirntod. Für das Transplantationsgesetz von 1995 musste eine Umschreibung gefunden werden, nach der der Mensch - zugespitzt formuliert - so tot wie nötig und die Organe so lebendig wie möglich sind.

Der Jurist Thomas Seibert erheiterte das Publikum mit seiner Deutung des Zwangs zur Abgabe der Steuererklärung als Freiheitsberaubung: das Formular, mit dem sie abgegeben werden muss, strukturiert die möglichen Antworten bereits vor und macht bestimmte Antworten unmöglich. Erst im Gerichtsverfahren besteht die Möglichkeit zur freien Erzählung der Sachverhalte.

\section{Theorien und Folien}

Am Nachmittag des ersten Tages wurden Detailuntersuchungen zu Gerichtspraxis und -verfahren vorgestellt. Johannes Schwitalla aus Würzburg untersuchte fingierte Gerichtsverhandlungen in Serien im ZDF und auf Sat 1. 
Er konnte aufzeigen, dass sie in Verfahrensablauf und -dauer sowie in der benutzten Sprache von der Wirklichkeit abweichen. Anstelle von Einigungsverhandlungen, die sich in Wirklichkeit oft quälend lange hinziehen, kommt es in den dramaturgisch aufbereiteten Sendungen bereits nach kurzen Verhandlungen zu einer Einigung. Das Auftreten der Personen und ihr Sprachgebrauch wirken insgesamt aufgesetzt. Wie eine Verhandlung abläuft, vermitteln die Serien daher nicht; eine „Botschaft“ liegt darin, dass eine Konfliktlösung immer als möglich angesehen wird.

An realen Fällen stellte Ralph Christensen zwei Ansätze der Rechtsanwendung vor. In einem Flugblatt zum 10. Jahrestag des Oktoberfest-Attentats von 1991 wurden Parallelen zwischen dem Verhalten der bayerischen Staatsregierung und dem, „was Hitler auf seine Fahnen geschrieben hatte“, gesehen. In der ersten Instanz wurden diejenigen, die das Flugblatt verfasst hatten, wegen Verunglimpfung des Staates und seiner Symbole nach $\S 90 \mathrm{a}$ StGB verurteilt, mit der Begründung, dass in dem Flugblatt eine Gleichsetzung des Freistaats Bayern mit dem faschistischen Unrechtsstaat Hitlers vorgenommen wurde. In der zweiten Instanz erfolgte ein Freispruch, da der Text des Flugblatts für das Gericht keine ausdrückliche Gleichsetzung enthielt und damit durch das Grundrecht der freien Meinungsäußerung abgedeckt war.

Christensen geht davon aus, dass jeweils eine von zwei impliziten Sprachtheorien das juristische Urteil bestimmt. Dem erstinstanzlichen Urteil lag die "starke" Theorie der Sprache zugrunde. Nach ihr steht alles Relevante im Gesetz, und ein Verfahren ist eigentlich nicht mehr nötig. Starken Einfluss auf das Urteilen haben hierbei die Gesetzeskommentare, die Beispiele für abstrakt gefasste gesetzliche Bestimmungen enthalten. Im geschilderten Fall wurde im Kommentar eine Umschreibung des Tatbestands gegeben, die im Urteil ohne weitere Begründung als Inhaltsangabe des Flugblatts übernommen wurde.

Dem Freispruch lag die „schwache“ Theorie der Sprache zugrunde: das Gesetz - plus Kommentar - gibt hiernach nicht objektiv vor, wann der Sachverhalt vorliegt. Vor Gericht tragen vielmehr alle Beteiligten ihre Sichtweise vor, und das Gericht entscheidet nach Anhörung und Abwägung aller Argumente. In der Urteilsbegründung muss das Gericht nicht den Kommentar wiederholen, sondern darlegen, dass es den besseren Argumenten folgt. Dies lässt im fraglichen Fall mehrere Lesarten des Flugblatts zu, also auch die, dass keine Gleichsetzung gemeint war. Normativität wird nach der schwachen Theorie nicht vorgefunden, sondern erst im Verfahren hergestellt.

$\mathrm{Ob}$ die beiden Ansätze in der Praxis nebeneinander existieren oder ob in der Praxis nicht eine „schwächliche“ Theorie der Empirie vorherrscht, wurde in der Diskussion aufgeworfen. Bereits das „Bullenbeispiel“ am Vortag hatte gezeigt, dass Richter und Richterinnen in der Praxis nicht nur in Gesetze oder Wörterbücher sehen. Auch dass es möglich ist, über mehrere Instanzen in Revision zu gehen und höhere Gerichte immer mit mehreren 
Richtern und Richterinnen besetzt sind, ist ein Indiz dafür, dass im deutschen Rechtssystem Rechtsanwendung nicht einfach der starken Theorie einer objektiven Bedeutung folgt.

In Gerichtsverfahren ist somit die Art der Tathergangs- bzw. Sachverhaltsbeschreibung entscheidend. Dies machte Gisela Harras aus sprachphilosophischer Sicht klar, indem sie nach den Merkmalen bzw. Bedingungen von Handlungen und Nicht-Handlungen in der Alltagssprache fragte. Ob z. B. „Vergessen, eine Kerze auszulöschen“ als nicht strafrelevante Handlung gewertet werden kann, hängt weitgehend von der Akzeptanz bestimmter Normen ab.

Auch der Verlauf eines Verfahrens wird von sogenannten Normalitätsfolien bestimmt, die Ludger Hoffmann in verschiedenen Anhörungen vor Gericht untersucht hat. Weicht eine Vorgangsschilderung vom Erfahrungswissen der Zuhörenden und deren Erwartungen ab, hinterfragen sie es. Dies beeinflusst beispielsweise die Befragung dahingehend, dass man Angeklagten nicht glaubt. Wenn Angeklagte jedoch ihr Verhalten in eine Normalitätsfolie einordnen (,,ich bin schließlich verheiratet und habe ein Kind, ich würde das nie tun"), haben sie eher eine Chance, glaubhaft zu erscheinen. Dass derartige Folien auch durch interkulturelle Missverständnisse und Vorurteile beeinflusst sein können, zeigte Jochen Rehbein an Verhandlungen im Rahmen von Abschiebeverfahren auf.

Christoph Sauer aus Groningen knüpfte an diese Untersuchungen an. Für ihn geht der Kampf im Gerichtsaal um Plausibilität. Dazu werden für die zahlreichen Aussagen, die im Laufe eines Verfahrens vorgeschrieben sind, gezielt die Inhalte und sprachlichen Mittel ausgewählt, die das Verständnis der Zuhörenden sichern. Der als Zeuge gehörte Polizist wird eher eine Berichtsform, der Angeklagte eher einen persönlichen Erzählstil wählen. Neben dem Ziel, Verständnis über den Vorgang zu erlangen, werden in Schattendiskursen auch andere Ziele verfolgt: wer Reue zeigt, kann manche Unstimmigkeiten überspielen und die Chancen auf ein mildes Urteil erhöhen.

\section{Rechtsprechen als Textarbeit}

Der Mittwoch begann mit zwei grundlegenden Vorträgen zur juristischen Textauslegung. Der ehemalige Verfassungsrichter Paul Kirchhof stellte Überlegungen zum Zusammenhang von Rechtsetzung, Sprache und den kulturellen Grundlagen des Staatsvolks an. Recht lebt in und von Sprache: es basiert auf guten Gewohnheiten, die schriftlich festgehalten werden. Die heutige Rechtssprache ist nüchtern, was zwar eine Distanz zum Staatsvolk mit sich bringt, aber damit zugleich Objektivität, Verlässlichkeit und Verbindlichkeit sichern soll.

Doch dies lässt sich nicht immer garantieren. Völlig ohne werbende Passagen kommen beispielsweise Verfassungen nicht aus und Bereiche, die noch nicht lange gesetzlich geregelt sind, wie der Naturschutz, suchen ihre 
präzise Gesetzessprache erst noch. Vieles, wie „Schule“, „Steuern“, „Partei““ scheint klar, ist aber aus alten Verfassungen übernommen und muss fortgebildet werden. In anderen Bereichen müssen gültige Normen neuen Anforderungen gerecht werden: „Presse“ ist heute nicht mehr nur das, was aus dem Pressstock kommt, und das Rundfunkgesetz gilt längst nicht mehr nur fürs Radio. Ähnliches gilt für veränderte gesellschaftliche Normen: Eine rauchende Frau war noch vor 100 Jahren eine Gefährdung der „öffentlichen Ordnung“, heute hat niemand mehr mit dieser Rechtsauffassung Erfolg vor Gericht. Festzustellen, ob etwas unter eine gesetzliche Regelung fällt oder nicht, bleibt daher in letzter Instanz immer Aufgabe des Gerichts. Es muss zu einem Urteil kommen, da jeder Bürger und jede Bürgerin einen Anspruch auf einen Urteilsspruch hat.

Der Referent sitzt einer Expertenkommission vor, die das deutsche Steuerrecht systematisiert. Bisher sind der Arbeitsgruppe ca. 170 Gesetze bekannt, die mit dem Steuerrecht zusammenhängen. Wie viele man am Ende finden wird, wollte er nicht abschätzen. Die Steuerzahler und -zahlerinnen werden bei der Abgabe ihrer Steuererklärung - so griff Kirchhof ein bereits am Vortag gestreiftes Thema auf - planmäßig überfordert. Was in der Steuererklärung an Angaben verlangt wird, ist derart abgehoben von der Alltagssprache, dass es nicht mehr verstanden werden kann. Wer beispielsweise glaubt, seine Ehe sei keine Zugewinngemeinschaft, weil es keinen materiellen Gewinn gab, Gütertrennung nicht ankreuzt, da dies angesichts des guten Klimas in der Ehe nicht angesagt ist und in der Steuererklärung dann Gütergemeinschaft angibt, gibt damit im Normalfall eine falsche Steuererklärung $a b$ - und das ist - auch wenn es auf Unkenntnis beruht - strafbar.

Dietrich Busse aus Köln betrachtete anschließend die juristische Auslegungstätigkeit aus linguistischer Sicht. Seine Kernaussage war, dass nicht ein einzelnes Gesetz bei der Beurteilung eines rechtsrelevanten Vorfalls Anwendung findet. Vielmehr produziert der Richter erst einen neuen Normtext, den er dann auf den vorliegenden Fall anwendet. Im Diebstahlsparagrafen des BGB beispielsweise ist von „Eigentum" oder „fremd“ die Rede. Was darunter zu verstehen ist, wird in anderen Gesetzen oder in Kommentaren erläutert. Dem Juristen oder der Juristin sind diese Regelungen bekannt. Die Verwendung von z.B. „Eigentum" ruft bei ihnen einen hochkomplexen und bedeutungskonstitutiven Wissensrahmen (,frame $<$ ) von Textgeflechten und dadurch die Bedeutung des Paragrafen auf. Juristen und Juristinnen wenden nicht einfach das Gesetz auf den vorliegenden Sachverhalt an, sondern stellen aufgrund ihres Textwissens erst alle einschlägigen Regelungen zusammen, bevor sie urteilen.

Für das abschließende Urteil ist oft nicht so sehr die Bedeutungsebene, sondern die Begründungsebene entscheidend. Kauft jemand beispielsweise ein gebrauchtes Auto mit fehlerhaften Reifen und kommt es zu einem Unfall, so könnten Gesetze verschieden angewendet werden. Erst aufgrund der Beschreibung des Vorgangs z. B. als Verkauf eines betriebsbereiten Autos 
oder eines nicht betriebsbereiten Autos - findet das eine oder andere Gesetz Anwendung.

\section{Sprache vor Gericht}

Die Forensik bestimmte den Donnerstag Morgen. Welches Handlungsmuster abläuft, wenn jemand eine Person - zum Beispiel durch die Bezeichnung als „Bulle“ - beleidigt, analysierte Hans Jürgen Heringer. Er beobachtete die Beteiligten, die Art der Formulierung und untersuchte die Frage, wann eine Beleidigung erfolgreich ist sowie deren Konsequenzen. Wenn eine Beleidigung erfolgreich ist, also jemand sich durch jemanden - sei es durch eine Äußerung oder durch Gesten - beleidigt fühlt, kann diese Person die Justitz einschalten. Sie verfolgt auf Antrag den Fall und verhängt eine Strafe, normalerweise eine Geldstrafe. Interessant waren die „Preisunterschiede" für ähnliche Beleidigungen in unterschiedlichen Urteilen.

Mit Erpresserbriefen befasste sich Christa Baldauf vom BKA aus Wiesbaden. Mit Beispielen konnte sie kaum dienen, da das BKA-Corpus streng unter Verschluss gehalten wird. Baldauf geht davon aus, dass es zwar keinen „sprachlichen Fingerabdruck“, aber einen Fingerzeig gibt und je mehr man über Sprache weiß, desto besser wird man ihn verstehen. Die linguistische Analyse kann z. B. Sprachgenese bedingte Fehler erfassen; Fehlerprofile und Ausdrucksweisen lassen auch bei Verstellung Rückschlüsse auf bestimmte Personenkreise oder Denkkonzepte zu. Auch Schreiben von Trittbrettfahrern kann man durch einen sprachlichen Vergleich mit Tätertexten identifizieren.

Für Norbert R. Wolf aus Würzburg gibt es ebenfalls keinen sprachlichen Fingerabdruck, da letztlich alle Aussagen über den Stil eines Textes impressionistisch sind. Wolf verwies auf einige Prozesse, in denen linguistische Gutachten sich im Nachhinein als falsch erwiesen. Zwar kann man an einzelnen Texten typische syntaktische Merkmale oder Worthäufungen feststellen, doch sind diese vor allem textsortentypisch oder situationsbedingt und keine individuellen Merkmale. Auch der Vergleich mit vermeintlich von derselben Person geschriebenen Texten, führt nicht zu sicheren Ergebnissen, da Erpresser oft den eigenen Sprachstil verstellen und die herangezogenen Vergleichstexte oft älter als der Erpresserbrief sind.

Recht und Sprache in der juristischen Ausbildung

Sprachprobleme in der juristischen Ausbildung und Rechtsprechung als Sprachproblem wurden am Mittwoch Nachmittag und Donnerstag Vormittag behandelt.

In der EU gibt es offiziell elf Amtssprachen und somit elf amtliche Versionen von Unionsgesetzen. Petra Braselmann aus Innsbruck zeigte anhand eines Falls, der vor dem Europäischen Gerichtshof verhandelt wurde, dass dies zur Folge haben kann, dass ein Gesetz in einer deutschen Fassung angewandt wird, die in Details von der französischen oder englischen Urfassung abweicht. Das Urteil lautet dann zu Ungunsten einer Klägerin, die als 
Französin evtl. Recht bekommen würde. Zwar sollten mögliche Übersetzungsfehler im Vorfeld linguistisch ermittelt und ausgeschaltet werden, aber gänzlich vermeiden lassen sie sich nicht. Daher wäre es besser, die Urfassung im Zweifelsfall für verbindlich zu erklären.

Für Gerard-Réne de Groot aus Maastricht ist das Übersetzen von Gesetzen nicht nur eine sprachwissenschaftliche Angelegenheit, sondern immer auch angewandte Rechtsvergleichung. $\mathrm{Zu}$ beachten ist hierbei auch, dass es für einige Sprachen mehrere Rechtssprachen gibt. Hinzu kämen noch die Übersetzungen für die deutschen Rechtssprachen der Schweiz, Liechtensteins, Italiens und Belgiens. Viele Übersetzungen sind auch nur teiläquivalent oder nicht umkehrbar, da die Rechtssysteme sich zu stark unterscheiden. In den Niederlanden beispielsweise können auch zwei Männer eine „Ehe“ eingehen: Die niederländische Bezeichnung für „Ehe“ unterscheidet sich semantisch also erheblich von der in Deutschland gebrauchten Bezeichnung. Nach de Groot sind viele der Wörterbücher, die er untersucht hat, in dieser Hinsicht äußerst unzulänglich.

Eine Konsequenz aus diesen Erkenntnissen wäre die Förderung von Mehrsprachigkeit und internationalem Recht unter Jura-Studierenden. Doch Wilhelm Grießhaber aus Münster und Gerhard Struck aus Hamburg wussten zu berichten, dass das Interesse der Jura-Studierenden an Sprachkursen nicht darauf gerichtet ist, sich Fremdsprachen anzueignen oder sich in fremde Rechtssysteme einzuarbeiten. Dies geschieht eher durch Praktika vor Ort. Erschwerend kommt hinzu, dass die sprachliche Ausbildung im deutschen Jura-Studium eher mit sprachlicher Dressur als mit Fachsprachenausbildung $\mathrm{zu}$ vergleichen ist und auf dieser Grundlage kein Interesse an kontrastiver Sprachbetrachtung geweckt wird.

\section{Musterland Schweiz?}

Am Mittwoch Vormittag unterhielt Theodor Stemmler aus Mannheim das Publikum mit verunglückten Formulierungen aus deutschen Landesverfassungen. So gewährt die bayerische Verfassung allen Bürgern und Bürgerinnen die „Aneignung wildwachsender Waldfrüchte in ortsüblichem Umfang“, gesunde Kinder sind „das köstlichste Gut eines Volkes“, in Bremen werden Richter vor das Gericht ,gezogen“, und die Syntax der Präambel der Verfassung von Baden-Württemberg ist so kompliziert, dass man sie erst nach dem dritten Lesen versteht. Die meisten „Pannen“ ergeben sich nach Stemmler aus einer verunglückten Syntax und unpassender Wortwahl, vor allem durch onomasiologische Fehlleistungen.

In der Schweiz wird man derlei verunglückte Formulierungen vermutlich nicht finden. Dort gibt es für deutschsprachige Gesetze eine siebenköpfige hauptamtliche Redaktionskommission, die an Gesetzgebungsverfahren beteiligt werden muss. Werner Hauck, Vorsitzender der deutschen Sektion der zentralen Sprachdienste der Bundeskanzlei, erläuterte, wie Gesetze unverständlich werden und wo die linguistische Arbeit beginnt. Entscheidend ist, 
dass der Text von Anfang an mit der Kommission gemeinsam erarbeitet und seine Verständlichkeit in allen Phasen des Verfahrens geprüft wird. Formulierungen sollten für die Zielgruppe des Gesetzes verständlich sein und in verschiedenen Gesetzen sollte Gleiches gleich benannt werden - ein Aspekt, der oft vergessen wird, wenn verschiedene Instanzen mit derselben Sache befasst sind. Er warnte vor dem Wahn, alles bis ins Detail abschließend im Gesetz zu regeln - dies sei eine Beamtensicht, und nicht die Perspektive der Rechtsbetroffenen. Mit Beispielen aus der Erarbeitung der neuen Schweizer Bundesverfassung zeigte Markus Nussbaumer, dass es der Kommission nicht nur um gelungene Formulierungen und klare Gliederung von Artikeln geht. Gesetze sind ein Arm der Politik, und viele Gesetze - besonders eine Verfassung - enthalten neben klaren Regelungen immer auch Ziel- oder Zweckbestimmungen. Diese können ,politisch unruhig" sein, d.h. es gibt keine einheitliche Interpretation des Ausdrucks, evtl. wird er als Fahnenwort sehr unterschiedlich verstanden. Seit 1996 nennt die Schweiz in ihrer Bundesverfassung als ein Staatsziel „Nachhaltigkeit“ - doch dieses Wort ist juristisch noch nicht ,stillgelegt", um seine Bedeutung wird politisch noch gestritten. Gerade in Verfassungen lassen sich derartige Wörter nicht vermeiden - sie sind politisch wünschenswert, um Themen in der Diskussion zu halten. Die Kommission kann aber durch die Nennung wichtiger Bestimmungselemente eine „Reliefgebung“ vornehmen.

Die Wechselwirkungen zwischen politischer Sprache und dem in Gesetzen fixierten Wortschatz machte Josef Klein an zahlreichen Beispielen aus der Kommunikation im deutschen Bundestag deutlich. Der parlamentarische Diskurs wirkt wie ein Transmissionsriemen, durch den - durchaus sehr zeittypische - begriffliche Orientierungen aus der politischen Allgemeinsprache in die Rechtssprache transportiert werden. Das Ergebnis scheint hierzulande, wo die Begleitung der Gesetzesformulierung durch eine Kommission fehlt, ungesteuerter und „wilder" als in der Schweiz.

\section{Geschichte}

In vielen Beiträgen wurde auf den Zusammenhang von Recht, Sprache und Kultur hingewiesen. Der historische Wandel dieses Zusammenhangs wurde in einem eigenen Block behandelt. Ingo Warnke aus Kassel stellte die Veränderungen im Übergang zur frühen Neuzeit und ihre Auswirkungen auf Recht und Rechtssprache dar; Hans Hattenhauer aus Kiel erläuterte, welche Auffassungen man seit dem 19. Jahrhundert mit „Rechtsstaat“ verband.

Heidrun Kämper zeigte anhand von Urteilen aus der Nachkriegszeit den Wandel in der Beurteilung und der Haltung zu NS-Verbrechen auf. 1947 war die Behauptung, man habe nicht alle Erlasse gekannt und nicht genau gewusst, wozu man beiträgt, unerheblich für die Strafbemessung. In späteren Urteilen entlastet Nichtwissen vom Vorwurf des Vorsatzes und wirkt strafmildernd. Parallel dazu wurde durch die Einführung der Kategorien „Haupttäter“ und „Mitläufer“ eine Schuldverlagerung auf einige wenige - meist tote - 
Mörder vorgenommen und andere Beteiligte mit dem Hinweis, sie hätten Gesetze befolgen müssen, entschuldigt. Die Schulddefinition hat sich gewandelt. Die Urteilsbegründungen belegen den Wandel der Schuldkonzepte und des Umgangs mit den NS-Verbrechen. Die Vorstellung eines naturrechtlichen Unrechts, die anfangs der Verurteilung zugrunde lag, wurde durch die positivistische Auffassung, man könne Angeklagte nur nach den damaligen Gesetzen verurteilen, ersetzt - moralisches Unrecht wurde zu juristischem Recht. Die Urteilsbegründungen sind daher in ihrer Widersprüchlichkeit besonders interessante Dokumente für die gesellschaftliche Bewertung der NS-Zeit.

\section{Kann man Gesetze verständlich machen?}

Das Abschlusspodium stand unter der Fragestellung „Kann man Gesetze verständlich machen?."

Nach Ingeborg Lasser aus Potsdam kann man Gesetze dann verständlich machen, wenn man an Rezeptionsbedingungen und Textfaktoren ansetzt. Große Hoffnung setzte sie in die übersichtlichere Gestaltung der Textoberfläche. Bereits Überschriften und andere Gliederungsmerkmale wie das Absetzen einzelner Elemente bei Aufzählungen machen einen Gesetzestext verständlicher. Im Internet kann man z. B. durch erläuternde Links Kommentar und Gesetz verknüpfen. Gesetze im Internet sieht man sich auch eher an als in gedruckter Form. Doch auch das Lesen von Gesetzen muss geübt werden. Lasser plädiert dafür, Lesestrategien schon in der Schule zu vermitteln. (Allerdings musste sie hier für Baden-Württemberg gleich Pessimismus verbreiten: die neue Prüfungsordnung für das Lehramt an Gymnasien sieht eine weitere Kürzung der sprachwissenschaftlichen Anteile vor.)

Ulrich Karpen, Jurist und Vorsitzender des Rechtsausschusses der Hamburger Bürgerschaft, forderte, dass Gesetze überschaubar sein müssen um zumindest von denjenigen, an die sie gerichtet sind, verstanden zu werden. Denn, wenn Recht nicht verstanden wird, wird es nicht akzeptiert, nicht mehr befolgt und damit wird der Rechtsstaat gefährdet.Vor allem sollen Gesetze nicht zu lang und gut verständlich sein. Bei der Abstimmung über Gesetze allerdings, so belegte er mit unterhaltsamen Beispielen, geht es nicht um Verständlichkeit. Wenn die Opposition die Verständlichkeit eines Gesetzes in Frage stellt und bessere Formulierungen vorschlägt, macht sich die Regierungsmehrheit diese normalerweise nicht zu Eigen, sondern stimmt für den eigenen ggf. unverständlichen Entwurf. Als Beispiel für unverständliche Gesetze führte er einige Paragrafen des auf der Tagung öfter bemühten Einkommenssteuergesetzes an. Auf die Frage, ob denn alle Mitglieder einer Fraktion ein Gesetz verstanden haben, dem sie zustimmen, erwiderte er, dass sich Abgeordnete jeweils in verschiedene Bereiche einarbeiten. Für diese Bereiche haben sie eine hohe Kompetenz und können auch Gesetzesentwürfe beurteilen. Für andere Bereiche übernehmen sie die Voten ihrer Fraktionskollegen und -kolleginnen.

Barbara Wieners-Horst aus Bonn, ist der „Redaktionsstab“ der Gesell- 
schaft für deutsche Sprache (Wiesbaden) beim deutschen Bundestag. Sie verkörpert den Stellenwert, der der gesetzgeberischen Sprachpflege in Deutschland verglichen mit der Schweiz zugestanden wird. Beim Bundestag gibt es nur ihre halbe Stelle, die in sprachlichen Fragen konsultiert werden kann. Ihre Möglichkeiten, die Formulierung von Gesetzen zu beeinflussen, sind daher gering. Sie konzentriert sich primär auf lexikalische und syntaktische Verstehenshindernisse. Oft machen Satzlänge und komplexe Nominalgruppen Gesetzesentwürfe unverständlich - sogar für die, die sie verfasst haben. Werner Hauck aus Bern ergänzte die Ausführungen aus seiner Praxis. Die Sprachwissenschaft müsse, so sein Aufruf am Ende, politischer werden und den Marsch durch die Institutionen antreten, sie dürfe sich nicht nur beklagen, sondern müsse „Anwälte der Verständlichkeit“ ausbilden.

Ansatzpunkte für linguistische Arbeit und rechtslinguistische Tätigkeitsfelder gibt es sicher mehr, als den meisten Teilnehmenden zu Beginn der Tagung bewusst war.

\subsection{Kolloquien/Workshops am IDS}

6.2.2001, GAIS - Gesprächsanalytisches Informationssystem, Kolloquium 5.-7.4.2001, Sprachliche Integration von Aussiedlern in den 90er Jahren Forschungsbilanz und Forschungsperspektiven, Internationales Kolloquium 26.6.2001, Tendenzen der Lexikalisierung kommunikativer Konzepte, Kolloquium

29.6.2001, „Wissen über Wörter“ - linguistische Konzeption und Struktur eines lexikalisch-lexikologischen Informationssystem, Kolloquium

16.-17.11.2001, Reflexivierung, Workshop

\subsection{Vorträge externer Wissenschaftler im IDS 2001}

20.04.2001, Prof. Dr. Rudolf Wille/TU Darmstadt, „Mensch und Mathematik. Logisches und mathematisches Denken“"

03.05.2001, Prof. Dr. Heide Wegener/Universität Potsdam, ,Pizzen und Kekse - Zur Assimilation von Fremdwörtern an das deutsche Flexionssystem“

15.06.2001, Prof. Dr. Peter Auer/Universität Freiburg, „Syntaktische Subordination und die Linearität der gesprochenen Sprache: Ein Widerspruch?““

15.06.2001, Prof. Dr. Ludwig Eichinger/Universität Kiel, ,,Syntaktische Präferenzen in informativen Texten. Zum Wandel von Textsortenerwartungen“ 15.06.2001, Prof. Dr. Hartmut Günther/Universität Köln, ,Stolz darauf, ein (z.B.) Germanist zu sein“

15.06.2001, Prof. Dr. Ludger Hoffmann/Universität Dortmund, „Funktionale Syntax" 
15.06.2001, Prof. Dr. Richard Wiese/Universität Marburg, „Zur Konversion im Deutschen“"

25.06.2001, Prof. Dr. Bernd Wiese/Berlin, „Zum Bau der Flexionsparadigmen des Neuhochdeutschen aus formbezogener Sicht"

13.07.2001, Prof. Dr. Anatolij Anatol'evič Polikarpov, „Principles of Computational Corpus Classification and Analysis: Russian Newspaper Texts of the End of the $20^{\text {th }}$ Century"

21.08.2001, Prof. Dr. Chang San-lii/Taiwan, „Inkongruenz der Handlungsinterpretationen in interkultureller Kommunikation“

05.11.2001, Dr. Renate Raffelsiefen/Hamburg, „Lücken und Paradigmaeffekte in der Flexionsmorphologie des Schwedischen"

\section{Lehraufträge und Vorträge von IDS-Mitarbeitern}

\subsection{Lehraufträge}

\section{Dr. Jannis Androutsopoulos}

SS 2001, Analyse gesprochener Sprache, Seminar, Pädagogische Hochschule Heidelberg

WS 2001/2002, Sprachvariation in der Medienkommunikation, Proseminar, Germanistische Linguistik und MKW, Universität Mannheim

\section{Michael Beißwenger}

SS 2001, Einführung in die Linguistik, Proseminar, Germanistisches Seminar, Universität Heidelberg

SS 2001, Einführung in die Sprachwissenschaft, Seminar, Institut für Germanistik, Universität Koblenz

WS 2001/2002, Online-Journalismus, Berufsbegleitende Weiterbildung, Akademie für Weiterbildung an den Universitäten Heidelberg und Mannheim e. V.

\section{PD Dr. Nina Berend}

WS 2001/2002, Deutsch in Mehrsprachigkeitssituationen, Hauptseminar, Universität Heidelberg

\section{PD Dr. Hardarik Blühdorn}

SS 2001, Kognitive Linguistik, Hauptseminar, Universität Halle-Wittenberg

WS 2001/2002, Teoria e análise da referência โReferenztheorie und Referenzanalyse], Hauptseminar, Universidade Federal do Rio Grande do Sul, Porto Alegre/Brasilien

\section{Dr. Elke Donalies}

5.6.-7.6.2001, Die deutsche Wortbildung, Hauptseminar als Blockseminar, Universität Köln 


\section{Prof. Dr. Reinhard Fiehler}

SS 2001, Sprache und Kommunikation im Alter, Blockseminar, Universität Bielefeld

\section{PD Dr. Claudia Fraas}

SS 2001, Kommunikation - Kultur, Strategien, Konflikte, Vorlesung, Universität Chemnitz

SS 2001, Medienwissenschaft, Proseminar, Universität Chemnitz

SS 2001, Text und Hypertext, Proseminar, Universität Chemnitz

SS 2001, Neue Medien - neue Kommunikations- und Informationsformen, Proseminar, Universität Chemnitz

WS 2001/2002, Kommunikation I, Vorlesung, Universität Chemnitz

WS 2001/2002, Einführung in die Medienwissenschaft, Proseminar, Universität Chemnitz

WS 2001/2002, Text und Multimedia, Proseminar, Universität Chemnitz WS 2001/2002, Neue Medien - neue Kommunikations- und Informationsformen, Proseminar, Universität Chemnitz

\section{Daniel Glatz}

WS 2001/2002, Deklarative Phonologie, Proseminar, Germanistisches Seminar, Lehrstuhl für Computerlinguistik, Universität Heidelberg

\section{Lutz Gunkel}

WS 2001/2002, Einführung in die deutsche Grammatik, Proseminar, Universität Heidelberg

\section{Prof. Dr. Gisela Harras}

WS 2001/2002, Übersetzen, Hauptseminar, Universität Mannheim

\section{Prof. Dr. Ulrike Haß-Zumkehr}

SS 2001, Lexikografie II: Wörterbücher schreiben, Hauptseminar, Universität Mannheim

WS 2001/2002, Einführung in die germanistische Linguistik (,Synchronie"), Vorlesung mit Tutorien, Universität Mannheim

\section{Prof. Dr. Werner Kallmeyer}

SS 2001 (zusammen mit Wilfried Schütte), Aspekte der Gesprächsforschung am Beispiel von Kommunikation über Kunst, Hauptseminar, Universität Mannheim

SS 2001 (zusammen mit Christine Bierbach), Linguistisches Kolloquium, Universität Mannheim

WS 2001/2002 (zusammen mit Wilfried Schütte), Gesprächsinszenierung im Fernsehen, Hauptseminar, Universität Mannheim

WS 2001/2002 (zusammen mit Christine Bierbach), Linguistisches Kolloquium, Universität Mannheim 


\section{PD Dr. Inken Keim}

WS 2001/2002, Einführung in die Soziolinguistik, Proseminar/Hauptseminar, Universität Mannheim

\section{Dr. Marek Konopka}

SS 2001, Linguistik, Vorlesung, Schule für Logopädie, Universitätsklinikum Heidelberg

WS 2001/2002, Linguistik, Vorlesung, Schule für Logopädie, Universitätsklinikum Heidelberg

\section{Dr. Anja Lobenstein-Reichmann}

SS 2001, Mittelhochdeutsch/Frühneuhochdeutsch, Proseminar, Universität Mannheim

WS 2001/2002, Mittelhochdeutsch, Proseminar, Universität Mannheim

\section{Prof. Dr. Katharina Meng}

SS 2001, Sprachpolitik, Sprachenpolitik und Minderheiten, Hauptseminar, Universität Mannheim

WS 2001/2002, Spracherwerb, Hauptseminar, Universität Mannheim

\section{PD Dr. Gereon Müller}

SS 2001, Flexion und Syntax des Nominals, Hauptseminar, Universität Tübingen

WS 2001/2002, Economy in Syntax, Hauptseminar, Universität Mannheim

\section{Dr. Herbert Schmidt}

SS 2001, Einführung in die diachrone Sprachwissenschaft, Einfuihrungsseminar, Universität Mannheim

SS 2001, Einführung in das Mittelhochdeutsche, Proseminar, Universität Heidelberg

WS 2001/2002, Einführung in die diachrone Sprachwissenschaft, Einführungsseminar, Universität Mannheim

WS 2001/2002, Einfuihrung in das Mittelhochdeutsche, Proseminar, Universität Heidelberg

\section{Dr. Rudolf Schmidt}

WS 2001/2002, Digitale Sprach- und Bildverarbeitung, Vorlesung, Berufsakademie Mannheim

\section{Dr. Wilfried Schütte}

SS 2001 (zusammen mit Werner Kallmeyer), Aspekte der Gesprächsforschung am Beispiel von Kommunikation über Kunst, Hauptseminar, Universität Mannheim 
WS 2001/2002 (zusammen mit Werner Kallmeyer), Gesprächsinszenierung im Fernsehen, Hauptseminar, Universität Mannheim

\section{PD Dr. Thomas Spranz-Fogasy}

SS 2001, Führungskräfte in den Medien, Hauptseminar, Universität Mannheim

WS 2001/2002, Einführung in die ärztliche Gesprächsführung, Kurs, Medizinische Fakultät, Universität Heidelberg

WS 2001/2002, Kommunikation von Führungskräften im Spiegel der Medien, Hauptseminar, Universität Saarbrücken

\section{Dr. Angelika Storrer}

WS 2001/2002, Online-Journalismus, Berufsbegleitende Weiterbildung, Akademie für Weiterbildung an den Universitäten Heidelberg und Mannheim e. V.

\section{Prof. Dr. Bruno Strecker}

WS 2001/2002, Die Struktur deutscher Nominalphrasen, Hauptseminar, Universität Augsburg

\section{Dr. Annette Trabold}

WS 2001/2002, Sprachgeschichte, Sprachkultur, Sprachkritik, Seminar, Pädagogische Hochschule Heidelberg

\section{Dr. Peter Wagener}

SS 2001, Advanced conversation and composition, Seminar, University of Wisconsin, Madison/USA

SS 2001, German dialectology, Vorlesung und Seminar, University of Wisconsin, Madison/USA

\section{Dr. Ulrich Hermann Waßner}

SS 2001, Semantische Relationen zwischen Sätzen, Hauptseminar, Institut für Deutsch als Fremdsprachenphilologie, Universität Heidelberg WS 2001/2002, Kausale Konnektoren - Kausale Relationen, Hauptseminar, Institut für Deutsch als Fremdsprachenphilologie, Universität Heidelberg

\section{Prof. Dr. Gisela Zifonun}

SS 2001, „Fremdwörter“ im Deutschen, Proseminar/Hauptseminar, Universität Mannheim

WS 2001/2002, Grundkurs Grammatik, Proseminar, Universität Mannheim 


\subsection{Kurse und Kurzseminare}

\section{PD Dr. Nina Berend}

17.10.2001, Interkulturelle Pädagogik, Workshop, Stadtjugendamt Mannheim

\section{PD Dr. Hardarik Blühdorn}

1.8.-3.8.2001, O papel da gramática no ensino e na aprendizagem de línguas estrangeiras - exemplificado na língua alemã [Die Rolle der Grammatik in Unterricht und Erwerb von Fremdsprachen - verdeutlicht am Beispiel des Deutschen], Seminar zur Lehrerfortbildung, VI Encontro de Professores de Línguas e Literaturas Estrangeiras EPLLE Internacional [VI. Internationaler Fremdsprachenlehrerkongress], Universidade Estadual Paulista UNESP, Assis/Brasilien

14.11.2001, Das Internet-Angebot des Instituts für Deutsche Sprache in Mannheim und seine Nutzung im Unterricht des Deutschen als Fremdsprache, Seminar zur Lehrerfortbildung, Goethe-Institut Porto Alegre/ Brasilien

\section{Dr. Eva Breindl}

8.1.2001, Das grammatische Informationssystem GRAMMIS: Wie schreibt man eine Hypertextgrammatik und wie benutzt man sie?, Hauptseminar Ewald Lang: Was sind und zu welchem Zweck schreibt man Grammatiken?, Humboldt-Universität Berlin

\section{Prof. Dr. Reinhard Fiehler}

28.9.2001 (zusammen mit A. Deppermann), Leitung der Sektion „Gesprächsforschung " zum Schwerpunktthema "Generationen im Gespräch" auf der Jahrestagung der GAL, Passau

1.11.2001, Workshop „Besonderheiten des Kommunikationsverhaltens im Alter", 1. Jahrestagung der Gesellschaft für Aphasieforschung und -behandlung, Bielefeld

\section{Prof. Dr. Gisela Harras}

1.-2.3.2001 (zusammen mit J. Grabowski), Leitung der AG „Dynamik der schriftlichen Sprachproduktion“, Jahrestagung der Deutschen Gesellschaft für Sprachwissenschaft, Universität Leipzig

\section{Prof. Dr. Dieter Herberg}

18.7.2001, Neologismen, Kurzseminar, Goethe-Institut Mannheim, IDS, Mannheim

\section{Prof. Dr. Werner Kallmeyer}

17.2.2001, Zur Arbeit mit großen Korpora, Doktorandenseminar, Romanistisches Seminar, Universität Basel/Schweiz 
9.5.2001 (zusammen mit Liisa Tiitula), Stereotype in der finnisch-deutschen Wirtschaftskommunikation, Kolloquium „Wirtschaftskommunikation“, Universität Tampere/Finnland

29.5.2001, Gesprächsrhetorik, Vorlesung, Fakultät für Linguistik und Literaturwissenschaft, Universität Bielefeld

5.7.2001, Zur Analyse von Interaktionsmodalitäten, Kolloquium „Interaktionsmodalitäten“, Universität Konstanz

25.7.-27.7.2001, Gesprächsrhetorik, Fortbildungsveranstaltung „Gesprächsführung“, BMI-Schule 1, Heimerzheim

13.12.2001, Sprachvariation und soziale Stile, Ringvorlesung „Sprachliche Variation", Philosophische Fakultät, Universität Mannheim

\section{PD Dr. Inken Keim}

10.9.-18.10.2001, Einführung in die neuere Soziolinguistik, Vorlesung, Weltsprachenuniversität Taschkent/Uzbekistan

10.9.-18.10.2001, Gesprächsanalyse am Beispiel von Gesprächen aus unterschiedlichen sozialen Milieus in Deutschland, Workshop, Weltsprachenuniversität Taschkent/Uzbekistan

\section{Ulrich Reitemeier}

1.4.-30.1 1.2001, Soziologie für Logopäden, Deuserschule, Ludwigshafen

\section{Dr. Wilfried Schütte}

7.3.2001, Kommunikation im Internet, insbesondere in E-Mails, Expertenhearing im Rahmen einer Projektwoche „Infoschul II - Telecommunication: past-present-future" und AG-Arbeit zu den Themen „Internetsprache" und "soziales Verhalten im Internet“, Heinrich-Hertz-Berufskolleg, Düsseldorf

\section{PD Dr. Thomas Spranz-Fogasy}

21.3.-23.3.2001, Vortragsmoderationen auf der 7. Arbeitstagung „Neuere Entwicklungen in der Gesprächsforschung“, Universität Freiburg 21.3.2001, Zur Rolle des Kontextes bei der Gesprächsauswertung, Workshop, 7. Arbeitstagung „Neuere Entwicklungen in der Gesprächsforschung", Universität Freiburg

14.6.2001, Koordinationstreffen der Gesprächsforschung/Pragmatik, IDS, Mannheim

30.6.2001, Communicating Citizenship in Decision Making Procedures, Datensitzung, Zentrum für interdisziplinäre Forschung, Bielefeld

\section{Prof. Dr. Gerhard Stickel}

1.6.-21.6.2001, Entwicklungstendenzen der deutschen Gegenwartssprache, Teil I und II, Kurzzeitdozentur an der Staatlichen Universität der Leningrader Region, St. Petersburg-Puschkin/Russland 


\section{Dr. Annette Trabold}

31.3.-1.4.2001, Workshop „Sprachkritik“, 10. Jahrestagung des Bulgarischen Deutschlehrerverbandes, Dobritsch/Bulgarien

\section{Dr.h.c. Klaus Vorderwülbecke}

23.2.2001, Landeskunde in Lehrwerk und Fernsehsprachkurs, Workshop, Internationale Lehrerfortbildung, Internationales Haus Sonnenberg, St. Andreasberg/Oberharz

\section{Dr. Peter Wagener}

18.1.-25.1.2001, Modern German: Language Shift, Change and Loss, Madison Area Technical College, Madison, Wisconsin/USA

12.5.2001, German in Wisconsin, Annual Max Kade Institute Mini-Conference on German Dialects in the Midwest, Madison, Wisconsin/USA

\subsection{Vorträge}

\section{Dr. Jannis Androutsopoulos}

7.2.2001, „Fette Bässe über Köpenick!“ Zur (medialen) Kommunikation in Musikszenen, Vortragsreihe der Gesellschaft für deutsche Sprache, Universität Heidelberg

9.3.2001, Konturen der SMS-Kommunikation am Beispiel einer Kleingruppe, 24. Stuttgarter Tage der Medienpädagogik, Stuttgart

31.3.2001, Switching from national language to English in media discourse: an analysis of German print and internet texts, Language, the Media and International Communication Conference, Oxford/England

2.6.2001, Schöne neue Schriftlichkeit: Jugendliche Schreibstile in der Gästebuch-Kommunikation, Internationale Fachkonferenz „Jugendsprachen - Spiegel der Zeit“, Universität Wuppertal

12.6.2001, Szenesprache - Szenekommunikation, Gastvortrag, Universität Mannheim

4.7.2001, Stilbildung im Internet, Gastvortrag, Universität Saarbrücken 14.9.2001, Linguistic variation in chat communication: code switching in Hellas, $5^{\text {th }}$ International Conference on Greek Linguistics, Paris/Frankreich

19.9.2001, Sprache in virtuellen Gemeinschaften, Vortragsreihe „Heilkunst und Sprache“, Zentrum für interkulturelle Psychologie, Heidelberg 29.9.2001, Vom „TechnoForum“ zum „Punk-Chat": Stilbildung im Internet, 32. GAL-Jahrestagung, Passau

3.10.2001, (zusammen mit Daniel Kraft), Homepage-Design: Problemlösungsstrategien in einer neuen Textsorte, Germanistentag 2001, Erlangen 27.10.2001, Sprachliche Kreuzungen im Alltagsleben Jugendlicher, Fachtagung des Amtes für rheinische Landeskunde, Köln

16.11.2001, Online-Gemeinschaften und sprachliche Variation, Workshop „Sprache und Kommunikation im Internet", Universität Hannover 
8.12.2001, Sprachliche Kreuzungen im Alltagsleben, „Viele Sprachen lernen ... ein nothwendiges Übel"? Chancen und Probleme der Mehrsprachigkeit, Universität Leipzig

\section{Michael Beißwenger}

16.11.2001 (zusammen mit Angelika Storrer), Chat-Kommunikation: Kommunikationstheoretische Verortung - sprachliche Besonderheiten Lust am Spiel, Workshop "Sprache und Kommunikation im Internet“, Universität Hannover

\section{Cyril Belica}

29.6.2001, (zusammen mit Kathrin Steyer), Die statistische Kookkurrenzanalyse/Korpusbasiertheit, Kolloquium „Wissen über Wörter“ - linguistische Konzeption und Struktur eines lexikalisch-lexikologischen Informationssystems, IDS, Mannheim

\section{PD Dr. Nina Berend}

31.3.2001, Deutsch-englischer Sprachkontakt in Sprachinseln in den USA, International Symposium on German Settlement Dialects: Sprachinselkonferenz 2001, Max Kade Center for German-American Studies, University of Kansas/USA

5.4.2001, Sprachliche Anpassung. Eine soziolinguistisch-dialektologische Untersuchung zum Russlanddeutschen, Internationales wissenschaftliches Kolloquium, Sprachliche Integration von Aussiedlern in den 90er Jahren - Forschungsbilanz und Forschungsperspektiven, IDS, Mannheim

20.7.2001, Vorüberlegungen zur Gestaltung einer Datenbank von Aussprachevarianten des Deutschen, Soziolinguistentag, Neuphilologische Fakultät, Universität Heidelberg

3.10.2001 (zusammen mit Rudolf Schmidt), Perspektiven der computergestützten Untersuchung der Variation im gesprochenen Deutsch, Deutscher Germanistentag 2001, Erlangen

\section{PD Dr. Hardarik Blühdorn}

13.3.2001, Die Adverbien hier, da, dort und ihre portugiesischen Entsprechungen, Internationales Kolloquium „Die kleineren Wortarten im Sprachvergleich Deutsch-Portugiesisch", Institute für Romanistik und Germanistik, Universität Rostock

22.5.2001, Zur Semantik des Raums am Beispiel der deiktischen Adverbien hier, da und dort, IDS, Mannheim

2.8.2001, Ensino e aprendizagem de línguas estrangeiras no começo do terceiro milênio - quo vadunt? [Lehren und Lernen von Fremdsprachen am Anfang des dritten Jahrtausends - Wohin geht der Weg?], VI Encontro de Professores de Línguas e Literaturas Estrangeiras [Hauptvortrag auf 
dem VI. Internationalen Fremdsprachenlehrerkongress EPLLE], Departamento de Letras Modernas, Universidade Estadual Paulista UNESP, Assis/Brasilien

26.9.2001, Lehren und Lernen von Fremdsprachen am Anfang des dritten Jahrtausends - Wohin geht der Weg?, Öffentlicher Abendvortrag zum Europäischen Tag der Sprachen, Vortragsreihe "Sprachforum" des IDS, Mannheim

11.12.2001, Raumadverbien und Demonstrativität. Am Beispiel des Deutschen, Germanistisches Institut, Universität Halle-Wittenberg

11.12.2001, Zur Semantik der deutschen Satzkonnektoren. Am Beispiel von wenn und denn, Hallesches Linguistenkolloquium, Philosophische Fakultät, Universität Halle-Wittenberg

\section{Franck Bodmer}

4.9.2001 (zusammen mit Rudolf Schmidt), Computertechnische Erschließung von Gesprächskorpora, GLDV-Workshop, Werkzeuge zur automatischen Analyse und Verarbeitung von Texten: Formate, Tools, SoftwareSysteme, Universität Trier

\section{Dr. Eva Breindl}

9.1.2001, Vom Buch zum Hypertext: Die „Grammatik der deutschen Sprache" (GDS) und das grammatische Informationssystem GRAMMIS, Humboldt-Universität Berlin

28.9.2001, Grammatik lernen mit Hypertexten, GAL-Tagung „Sprache Transdisziplinär", Passau

\section{Ibrahim Cindark}

30.11.2001 (zusammen mit Werner Kallmeyer und Inken Keim), Metalinguistische Intuition in der Analyse von sprachlichen Orientierungen in deutsch-türkischen Migrantengruppen, Kolloquium ,Metalinguistic Intuitions“ der Forschergruppe „Sprachvariation als kommunikative Praxis“, Universität Mannheim

14.12.2001 (zusammen mit Werner Kallmeyer und Inken Keim), Deutschtürkische Sprachvariation, Kolloquium „Variation and Contrast: Sociolinguistic and pragmatic perspectives on language in use", Universität Mannheim

\section{Prof. Dr. Reinhard Fiehler}

31.1.2001, Operator-Skopus-Strukturen in gesprochener und geschriebener Sprache, Universität Konstanz

15.2.2001 (zusammen mit Reinhold Schmitt), Kundenorientierte Gesprächsführung. Authentische Beispiele aus der Praxis und wie man es besser machen kann, 45. Ulmer Beton- und Fertigteil-Tage 20.4.2001, Syntaktische Besonderheiten gesprochener Sprache und ihr theoretisches Potenzial, Universität Rostock 
18.5.2001, Bewertung von Kommunikationsverhalten - im Kontext von Kommunikationsberatung und -training, 29. Treffen des Arbeitskreises Angewandte Gesprächsforschung, Universität Wien

27.9.2001, Die kommunikative Verfertigung von Altersidentität, 32. Jahrestagung der GAL, Sektion ,Gesprächsforschung', Passau

12.10.2001, Mündlichkeit und gesprochene Sprache als wissenschaftlicher Gegenstand - Wo ist da das Problem?, Jahrestagung der Deutschen Gesellschaft für Sprechwissenschaft und Sprecherziehung, Landau 30.11.2001, Operator-Skopus-Strukturen im gesprochenen und geschriebenen Deutsch, Freie Universität Berlin

\section{PD Dr. Claudia Fraas}

19.9.2001, Kognition und gesellschaftliche Konstruktion der Wirklichkeit - Begriffsgeschichte zwischen Kognitivismus und Konstruktivismus, Tagung „Deutsche Sprachgeschichte nach 1945. Theoretische Grundlagen, methodische Möglichkeiten, empirische Forschungsergebnisse", Universität Düsseldorf

\section{Daniel Glatz}

26.6.2001, Zur Lexikalisierung ereignisstruktureller Eigenschaften bei Sprechaktverben, Kolloquium ,Tendenzen der Lexikalisierung kommunikativer Konzepte", IDS, Mannheim

29.8.2001, On some event structural pecularities of verbs of communication, 34 $4^{\text {th }}$ SLE Meeting: „Language Study in Europe at the turn of the Millenium“, Societas Linguistica Europaea (SLE), Katholieke Universiteit Leuven/Belgien

\section{Lutz Gunkel}

9.5.2001, Das System der infiniten Verbformen im Deutschen, Deutsche Gesellschaft, Universiät Kraków/Polen

10.5.2001, Das Passiv im Deutschen, Deutsche Gesellschaft, Universität Lódź/Polen

16.11.2001, Reflexivierung in A.c.I.-Konstruktionen, Workshop „Reflexivierung“, IDS, Mannheim

\section{Prof. Dr. Gisela Harras}

16.03.2001, Mord, Totschlag \& Handlung. Verursachung und Verantwortung in der Handlungssprache. 37. Jahrestagung des IDS, Sprache und Recht, Mannheim

26.06.2001, Ein konzeptuelles System für die Semantik von Sprechaktverben, Kolloquium „Tendenzen der Lexikalisierung kommunikativer Konzepte“, IDS, Mannheim

11.07.2001, Einige Merkwürdigkeiten der Semantik von Sprechaktverben, Universität Passau 
19.07.2001, Sprechakte und Sprechaktverben, Universität Trier

31.8.2001 (zusammen mit Kristel Proost), The Lexicalization of Speech Act Evaluations in English, Dutch and German, $34^{\text {th }}$ International SLE 2001 Conference „Language Study in Europe at the Turn of the Millennium: Towards the Integration of Cognitive, Historical and Cultural Approaches to Language", Katholieke Universiteit Leuven/Belgien

28.09.2001, Intentionalität als Kategorie der Handlungssprache, Jahrestagung der Gesellschaft für angewandte Linguistik, Universität Passau

\section{Prof. Dr. Ulrike Haß-Zumkehr}

20.9.2001, Hat die Frauenbewegung Wortschatzgeschichte geschrieben?, Abschiedskolloquium für Georg Stötzel „Sprachgeschichte nach 1945“, Düsseldorf

15.11.2001, Hermann Pauls „Principien der Sprachgeschichte“. Von der ersten zur zweiten Auflage, Internationale Konferenz „Geschichte der Sprachwissenschaft in Texten und Konzepten“, Universität Potsdam 17.11.2001, Das Projekt „Wissen über Wörter“, Tagung „Sprachkultur und Lexikographie. Von der Forschung zur Nutzung von Wörterbüchern“, Verein zur Förderung sprachwissenschaftlicher Studien e. V., Humboldt Universität Berlin

\section{Dr. Klaus Heller}

11.05.2001: Kommunikative Normen im Wandel, Statement zur Podiumsdiskussion „Sprache - Moden - Normen“, Universität Innsbruck

\section{Prof. Dr. Dieter Herberg}

22.3.2001, „Techno-Deutsch: Worthülsen statt Informationen?“, Tenovision Talk, Cebit, Hannover

17.11.2001, Das Projekt „Neologismen der 90er Jahre des 20. Jahrhunderts“, Tagung ,Sprachkultur und Lexikographie“, VfsS und HumboldtUniversität, Berlin

\section{Prof. Dr. Werner Kallmeyer}

29.6.2001 (zusammen mit Inken Keim und Ersoy Sentürk), How young Turks use their communicative repertoire, Workshop on „Modern Urban Bilingualism", Tilburg/Niederlande

28.9.2001, Kommunikative soziale Stile, GAL-Tagung „Sprache TRANSDISZIPLINÄR“, Universität Passau

1.10.2001, Kommunikative soziale Stile und wechselseitige Wahrnehmung sozialer Gruppen, Jahrestagung des Deutschen Germanistenverbandes, Universität Erlangen

25.10.2001, Zur pragmatischen Analyse von Mediensprache und Medienkommunikation, Konferenz „Mediensprache aus interdisziplinärerer Sicht", Philologische Fakultät, Lomonosov-Universität Moskau/Russland 
29.11.2001, Gesprächsrhetorik, Fachhochschule des Bundes für öffentliche Verwaltung, Haar

30.11.2001 (zusammen mit Inken Keim und Ibrahim Cindark), Metalinguistische Intuition in der Analyse von sprachlichen Orientierungen in deutsch-türkischen Migrantengruppen, Kolloquium „Metalinguistic Intuitions" der Forschergruppe „Sprachvariation als kommunikative Praxis“, Universität Mannheim

14.12.2001 (zusammen mit Inken Keim und Ibrahim Cindark), Deutschtürkische Sprachvariation, Kolloquium „Variation and Contrast: Sociolinguistic and pragmatic perspectives on language in use", Universität Mannheim

\section{Dr. Heidrun Kämper}

14.3.2001, „Übergesetzliches Recht“. Reflexionen nationalsozialistischen Unrechts in der frühen Nachkriegszeit, 37. Jahrestagung des IDS, Sprache und Recht, Mannheim

20.4.2001, Zeitreflexion und Sprachgeschichte. Ein Konzept zur Erforschung des Nachkriegsdeutschen, Tagung „Wissenschaftstheoretische Perspektiven einer künftigen Linguistik", Ascona, Monte Verità/Italien

21.9.2001, Zeitreflexion in der frühen Nachkriegszeit, Tagung „Deutsche Sprachgeschichte nach 1945“, Heinrich-Heine-Universität Düsseldorf

5.12.2001, „Es ist doch nirgendwo so interessant wie hier und heute“. Gegenwartsbewusstsein und Sprache in der frühen Nachkriegszeit, Vortragsreihe „Sprachforum“, IDS, Mannheim

\section{PD Dr. Inken Keim}

28.5.2001, Kommunikationsstile in jugendlichen MigrantInnengruppen, Kolloquium „Theorie und Praxis“, Gesamthochschule Kassel

31.5.2001 (zusammen mit Ibrahim Cindark), Der unterschiedliche Umgang mit dem negativen Face in zwei Gruppen jugendlicher MigrantInnen, Internationale Fachkonferenz, „Jugendsprachen - Spiegel der Zeit“, Wuppertal

29.6.2001 (zusammen mit Werner Kallmeyer und Ersoy Sentürk), How young Turks use their communicative repertoire, Workshop on „Modern Urban Bilingualism", Tilburg/Niederlande

5.7.2001, „Gespanntheit" zwischen Spiel und Gereiztheit. Einordnung des Konzepts "Gespanntheit“" und Exemplifizierung am Beispiel einer Migrantinnengruppe, Workshop „Interaktionsmodalitäten“, Universität Konstanz

30.11.2001 (zusammen mit Werner Kallmeyer und Ibrahim Cindark), Metalinguistische Intuition in der Analyse von sprachlichen Orientierungen in deutsch-türkischen Migrantengruppen, Kolloquium „Metalinguistic Intuitions" der Forschergruppe "Sprachvariation als kommunikative Praxis“, Universität Mannheim 
14.12.2001 (zusammen mit Werner Kallmeyer und Ibrahim Cindark), Deutsch-türkische Sprachvariation, Kolloquium „Variation and Contrast: Sociolinguistic and pragmatic perspectives on language in use", Universität Mannheim

\section{Dr. Anja Lobenstein-Reichmann}

6.10.2001, Luther und die Bauern. Haben die Bauern Luther missverstanden?, 25. Annual German Studies Association Conference, German Studies Association, Washington D.C./USA

\section{Prof. Dr. Katharina Meng}

5.4.2001, Sprachliche Integration von Aussiedlern - einige Ergebnisse, einige Probleme, Internationales wissenschaftliches Kolloquium, Sprachliche Integration von Aussiedlern in den 90er Jahren - Forschungsbilanz und Forschungsperspektiven, IDS, Mannheim

27.4.2001, Sprachliche Integration von Aussiedlern: Familie Sennwald, Tagung „Polyphonie in Kultur, Text und Sprache“, Lehrstuhl für russische Sprache, Universität Vilnius/Litauen

28.5.2001, Mehrsprachigkeit aus der Sicht der Familie, Tagung „Chancen und Probleme von Mehrsprachigkeit in der Grundschule“, Fachbereich Germanistik, Primarstufendidaktik, Universität Gesamthochschule Kassel 29.8.2001, Selbstbezeichnungen und kulturelle Selbstverständnisse bei Russlanddeutschen, Tagung „Language study in Europe at the turn of the millenium. Towards the integration of cognitive, historical and cultural approaches to language" $34^{\text {th }}$ SLE Meeting, Katholieke Universiteit Leuven/ Belgien

28.9.2001, Kulturelle Zugehörigkeiten als Gegenstand interkultureller Gespräche, Tagung „Sprache transdisziplinär“, 32. Jahrestagung der Gesellschaft für Angewandte Linguistik, Passau

28.10.2001, Russischsprachige Kinder in Deutschland: ihre familiäre Spracherwerbssituation, Tagung „Language Contact and Language Policy before and after the 1992 European Charter. Focus on Multilingual Domains (Administration, Education, Law and Media)", Seminar für Allgemeine Linguistik und Eurolinguistischer Arbeitskreis Mannheim e.V., Universität Mannheim

\section{PD Dr. Gereon Müller}

17.2.2001, The Deletion of Morphological Case, Fourth Workshop on Optimality Theoretic Syntax, Universität Stuttgart

1.3.2001, Free Word Order, Morphological Case, and Sympathy Theory, Tagung der Deutschen Gesellschaft für Sprachwissenschaft (DGfS), AG "Clause Structure and Models of Grammar from the Perspective of Languages with Rich Morphology", Universität Leipzig

24.4.2001, Was bringt die Optimalitätstheorie für die deutsche Grammatik?, Kolloquium, IDS, Mannheim 
17.6.2001, Freie Wortstellung, morphologischer Kasus und Sympathietheorie, Treffen „Generative Grammatik im Süden“, Universität Bochum 23.8.2001, Remarks on Nominal Inflection in German, SFB-Tagung „The Lexicon in Linguistic Theory“, Universität Düsseldorf 27.10.2001, V/2 as Remnant vP Movement, Workshop on Head Movement, University of California, Los Angeles/USA

7.11.2001, Deutsche Nominalflexion in der Optimalitätstheorie, Linguistischer Arbeitskreis, Institut für Deutsche. Sprache und Literatur, Universität Köln

7.12.2001, On Phrase Impenetrability, Workshop on the MLC, Universität Potsdam

13.12.2001, Remarks on Nominal Inflection in German, Optimality Theoretic Syntax Workshop, Universität Potsdam

\section{Kristel Proost}

26.6.2001, (Un)möglichkeiten der Lexikalisierung kommunikativer Konzepte, Kolloquium, „Tendenzen der Lexikalisierung kommunikativer Konzepte", IDS, Mannheim

31.8.2001 (zusammen mit Gisela Harras), The Lexicalization of Speech Act Evaluations in English, Dutch and German, $34^{\text {th }}$ International SLE 2001 Conference „Language Study in Europe at the Turn of the Millennium: Towards the Integration of Cognitive, Historical and Cultural Approaches to Language", Katholieke Universiteit Leuven/Belgien

28.9.2001, Lücken und Lückenfüller im Wortschatzausschnitt der kommunikativen Ausdrücke, 32. Jahrestagung der Gesellschaft für Angewandte Sprachwissenschaft, „Sprache transdisziplinär“, Universität Passau

\section{Ulrich Reitemeier}

5.4.2001, Aussiedler treffen auf Einheimische. Paradoxien der Identitätsarbeit und Schwierigkeiten des Aufbaus gemeinsamer kommunikativer Welten, Kolloquium „Sprachliche Integration von Aussiedlern in den 90er Jahren - Forschungsbilanz und Forschungsperspektiven“, IDS, Mannheim

27.11.2001, Perspektiven der sprachlichen Integration von Aussiedlern, Fachtagung für Sozialarbeit der Diakonie in Baden, „Starke Partner - gute Perspektiven. Gemeinsam in Haupt- und Ehrenamt“, Bad Herrenalb

\section{Dr. Rudolf Schmidt}

26.3.2001, Automatische Detektion von Aussprachevarianten in Korpora gesprochener Sprache, GESUS Jahrestagung, Universität Bochum 30.3.2001, Instrumente zur Erstellung multimedialer Gesprächskorpora, GLDV-Frühjahrstagung, Universität Gießen

4.9.2001 (zusammen mit Franck Bodmer), Computertechnische ErschlieBung von Gesprächskorpora, GLDV-Workshop, „Werkzeuge zur automa- 
tischen Analyse und Verarbeitung von Texten: Formate, Tools, SoftwareSysteme, Universität Trier

3.10.2001 (zusammen mit Nina Berend), Perspektiven der computergestützten Untersuchung der Variation im gesprochenen Deutsch, Deutscher Germanistentag 2001, Erlangen

\section{Dr. Reinhold Schmitt}

15.2.2001 (zusammen mit Reinhard Fiehler), Kundenorientierte Gesprächsführung. Authentische Beispiele aus der Praxis und wie man es besser machen kann, 45. Ulmer Beton- und Fertigteil-Tage

\section{Dr. des. Ulrich Schnörch}

29.6.2001, Lemma-Auswahl in WiW und konzeptionelle Perspektiven, Kolloquium „Wissen über Wörter“ - linguistische Konzeption und Struktur eines lexikalisch-lexikologischen Informationssystem, IDS, Mannheim

\section{Dr. Wilfried Schütte}

17.1.2001, „Sie ham die Stelle!“ Zur sozialen Welt der Orchestermusiker, Vortragsreihe „Sprachszenen, Szenesprachen“, Gesellschaft für Deutsche Sprache und Germanistisches Seminar, Universität Heidelberg

6.2.2001, GAIS - Gesprächsanalytisches Informationssystem, Kolloquium, IDS, Mannheim

11.5.2001, GAIS - Gesprächsanalytisches Informationssystem, PortaLingua-Projekttagung, Projektverbund „PortaLingua“ („Hochschulverbund zum Einsatz curricularer Bausteine in der Sprach- und Kommunikationswissenschaft - multimediale Lehr- und Lernressourcen für Präsenzlehre, Selbststudium und Weiterbildung“", Konsortialführer Universität Essen) im Rahmen des BMBF-Förderprogramms „Neue Medien in der Bildung“, Essen

5.6.2001, „Thanks for letting me rant off topic“. Zum kommunikativen Stil in professionell genutzten Mailinglisten und anderen Internet-Foren, Ringvorlesung „Homo s@piens - Arbeiten und Leben in der vernetzten Welt", Institut für Arbeitswissenschaft und Technologiemanagement, Universität Stuttgart

29.6.2001, IDS-Werkzeuge für Gesprächskorpora: DIDA als Editor für Transkriptpartituren und COSMAS-II als Recherchesystem, Workshop „Transkriptionen: Standards, Tools und gesprächsanalytische Anforderungen“, Graduiertenkolleg „Aufgabenorientierte Kommunikation“, Universität Bielefeld

28.9.2001, „TV Total“: Ein Fernsehformat zwischen Postmoderne, Genre-Mix und Marketing, 32. Jahrestagung der Gesellschaft für Angewandte Linguistik (GAL), ,Sprache transdisziplinär“", Sektion Medienkommunikation, Rahmenthema: „Das Vergnügen in und an den Medien“, Passau 
16.11.2001, Formen der Kommunikationsregulierung in professionell genutzten Mailinglisten, Workshop, ,Sprache und Kommunikation im Internet“, Projekt sprache@web, Universität Hannover

\section{Dr. Horst Schwinn}

12.5.2001, Vorstellung der ProGr@mm-Konzeption, Projektverbund PortaLingua, Universität Essen

\section{PD Dr. Thomas Spranz-Fogasy}

27.2.2001, Was Politiker reden - Der kommunikative Alltag von Führungskräften der Politik, 5. Arbeitstreffen des Arbeitskreises Linguistische Pragmatik, Universität Leipzig

3.5.2001, Ethnografie gesellschaftlicher Führungskräfte in der Bundesrepublik Deutschland, Fachhochschule Fulda

23.5.2001, Einführung in die ärztliche Gesprächsführung, Medizinische Fakultät der Universität Heidelberg

28.6.2001, Kommunikationsstilistische Eigenschaften gesellschaftlicher Führungskräfte im Spiegel der Medien, Sektion „Textlinguistik/Stilistik“, 32. Jahrestagung der Gesellschaft für Angewandte Linguistik (GAL), „Sprache transdisziplinär“", Universität Passau

30.6.2001, Communicative Involvement in Public Discourses as Paradigm for Citizenship in Decision Making Procedures, Zentrum für interdisziplinäre Forschung, Bielefeld

15.11.2001, Gesprächsanalyse als Instrument zur Untersuchung gestörter Kommunikation, Arbeitstagung „Gestörte Kommunikation“, Philosophische Fakultät, Universität Rostock

\section{Dr. Doris Steffens}

5.4.2001, Die Neologismen der 90er Jahre im Deutschen unter besonderer Berücksichtigung der Anglizismen, Tagung „Deutsche und Bulgaren im Gespräch“, Universität Sofia/Bulgarien

\section{Dr. Kathrin Steyer}

22.6.2001, Die korpusbasierte Kookkurrenzanalyse - ein empirischer Weg zur Verifizierung usueller Wortverbindungen, Internationale Phraseologietagung, „Phraseologie und Parömiologie - neue Perspektiven der Forschung", Ascona/Schweiz

29.6.2001, (zusammen mit Cyril Belica), Die statistische Kookkurrenzanalyse/Korpusbasiertheit, Kolloquium „Wissen über Wörter“ - linguistische Konzeption und Struktur eines lexikalisch-lexikologischen Informationssystem, IDS, Mannheim

\section{Prof. Dr. Gerhard Stickel}

26.1.2001, Die Entwicklung des Deutschen im mehrsprachigen Europa, Beitrag zum Symposium „,Wege zu einer europäischen Identität“, Auftakt- 
veranstaltung des Europäischen Jahres der Sprachen 2001, Goethe-Institut, München

1.2.2001, Die Bedeutung der eigenen Sprache im "global village“, 5. Deutsch-Niederländische Konferenz über „Sprache, Kommunikation und Identität im sich verändernden Europa“, Duitsland Instituut, Amsterdam/Holland

12.2.2001, Aktuelle Tendenzen der deutschen Sprache, Goethe-Institut Mailand/Italien

13.3.2001, Eröffnung der Jahrestagung „Sprache und Recht“, 37. Jahrestagung des IDS, Mannheim

24.3.2001, Anglizismen im Deutschen, Klett-Forum, Leipziger Messe

20.4.2001, Deutsch, Englisch und das vielsprachige Europa, Beitrag zu einer Vortragsreihe des Institut Cervantes auf der Expolingua, Madrid/Spanien

8.5.2001, Eigene und fremde Sprachen im vielsprachigen Europa, Ringvorlesung „Sprachen und Sprachpolitik in Europa“, Universität München 24.5.2001, Deutsch als Fachsprache in deutschen Forschungsinstituten, Konferenz der Sektion „Geistes- und Bildungswissenschaften“ der Wissenschaftsgemeinschaft Leibniz, Nürnberg

12.6.2001, Kontrastive Linguistik und Fremdsprachenunterricht, Staatliche Universität der Leningrader Region, St. Petersburg-Puschkin/Russland

14.6.2001, Pragmatik der Negation, Staatliche Universität der Leningrader Region, St. Petersburg-Puschkin/Russland

2.10.2001, Germanistik heute, Sprach- und wissenschaftspolitisches Podium. Germanistentag 2001, Erlangen

25.10.2001, Wie weit sind wir?, Eröffnungsvortrag zur Konferenz „Europa plurilingue - Projetti per la promozione delle lingue nazionali“", Academia della Crusca, Florenz/Italien

27.11.2001, Podium: „Daheim im Haus Europa - wo wohnt die deutsche Sprache?“, Internationale Konferenz der GfdS „Deutsch im vielsprachigen Europa“, Goethe-Institut Brüssel/Belgien

\section{Dr. Angelika Storrer}

16.1.2001, Computervermittelte Kommunikation, Institut für deutsche Sprache und Literatur, Universität Dortmund

28.6.2001, Hypertext und Textgrammatik, Symposium „Sprache und Kommunikation", TU Aachen

16.11.2001 (gemeinsam mit Michael Beißwenger), Chat-Kommunikation: Kommunikationstheoretische Verortung - sprachliche Besonderheiten Lust am Spiel, Workshop „Sprache und Kommunikation im Internet", Universität Hannover

1.12.2001, Deixis in der computervermittelten Kommunikation, Internationale Tagung „Funktionale Pragmatik“, Universität Köln 


\section{Prof. Dr. Bruno Strecker}

28.6.2001, Grammatik im Rahmen von Kommunikationsforschung, Symposium „Kommunikation/Sprache“, RWTH Aachen

\section{Eva Teubert}

27.11.2001, Das Institut für Deutsche Sprache und seine Bibliothek, Tagung des Arbeitskreises Bibliotheken der WGL, Forschungszentrum Rossendorf

\section{Dr. Annette Trabold}

19.1.2001, Öffentlichkeitsarbeit für die Wissenschaft, Workshop „Probleme fachexterner Kommunikation in der Öffentlichkeitsarbeit", Universität Koblenz-Landau

30.3.2001, Das Institut für Deutsche Sprache und aktuelle Entwicklungen der deutschen Sprache, 10. Jahrestagung des Bulgarischen Deutschlehrerverbandes, Dobritsch/Bulgarien

4.4.2001, Das Institut für Deutsche Sprache, Aufgaben und Ziele, ZontaClub, Mannheim

16.5.2001, Das Institut für Deutsche Sprache, Aufgaben und Ziele; Artes Liberales, Mannheim

\section{Dr. h.c. Klaus Vorderwülbecke}

19.3.2001, Einführung in den Fernsehsprachkurs „Einblicke“, Präsentation von GRAMMIS, Goethe-Institut Tel Aviv/Israel

21.3.2001, Einführung in den Fernsehsprachkurs „Einblicke“, Goethe-Institut Jerusalem

12.5.2001, Vorstellung der ProGr@mm-Konzeption, Tagung des Projektverbunds PortaLingua, Universität Essen

23.7.2001, Projekte und Simulationen in und zu Lehrwerken, Lehrerfortbildungsseminar des Goethe-Instituts Augsburg

2.8.2001, Sequenzieren Sie mal! (Phonetik), Internationaler Deutschlehrertag, Luzern/Schweiz

20.10.2001, Unterrichtsprojekte, Deutschlehrertag der „Regio“, Freiburg

\section{Dr. Peter Wagener}

31.1.2001, Languages in a Virtual World: German and German-American Dialects on the Internet, Max Kade Institute, University of Wisconsin, Madison/USA

31.3.2001, Wozu noch Deutsch? Funktionen und Funktionsverluste des Deutschen in Wisconsin, International Symposium on German, Settlement Dialects: Sprachinselkonferenz 2001, Max Kade Center for GermanAmerican Studies, University of Kansas, Lawrence/USA 10.4.2001, Sprachbiographien deutschstämmiger Emigranten und Aussiedler, German Department, University of Wisconsin, Madison/USA 
23.4.2001, Sprachverlust, 7. Germanic Linguistics Annual Conference, University of Alberta, Banff/Canada

26.4.2001, Corpus Linguistics in the Internet: German Dialects Virtual, Department of Foreign Languages, Iowa State, Ames/USA

16.10.2001, Databases of Spoken German, Linguistic Data Consortium, University of Pennsylvania, Philadelphia/USA

22.10.2001, Language Loss, Linguistic Laboratories, University of Pennsylvania, Philadelphia/USA

23.10.2001, Spoken German online: The virtual German Language Archives, Department of German and Slavic Studies, University of Georgia, Athens/USA

27.10.2001, Ist Niederdeutsch eine Minderheitensprache? Zur Sprachpolitik in Norddeutschland, $3^{\text {rd }}$ International Symposium on Eurolinguistics, Universität Mannheim

\section{Dr. Ulrich Hermann Waßner}

26.3.2001, Repetitive Konnektoren, Gesellschaft für Sprache und Sprachen e. V. (GESUS), Bochum

\section{Prof. Dr. Gisela Zifonun}

27.4.2001, Grammatik des Deutschen im europäischen Vergleich: Sprachtypologie und einzelsprachliche Grammatikographie, Germanistisches Institut, Universität Potsdam

27.9.2000, Grammatik des Deutschen im europäischenVergleich: Der Relativsatz, Wirtschaftshochschule Kopenhagen/Dänemark

\section{Im Berichtsjahr erschienene Publikationen von IDS-Mitarbeitern}

\section{Dr. Jannis Androutsopoulos}

Androutsopoulos, Jannis (2001): From the streets to the screens and back again: On the mediated diffusion of variation patterns in contemporary German. In: LAUD Linguistic Agency, Series A: No. 522. Universität Essen. $27 \mathrm{~S}$.

Androutsopoulos, Jannis (2001): Ultra korregd Alder! Zur medialen Stilisierung und Popularisierung von „Türkendeutsch“. http://www.linse.uniessen.de/papers/ultra_korregd/ultra_korregd.pdf

Androutsopoulos, Jannis/Hinnenkamp, Volker (2001): Code-Switching in der bilingualen Chat-Kommunikation: ein explorativer Blick auf hellas und turks. In: Beißwenger, Michael (Hg.): Chat-Kommunikation: Sprache, Interaktion, Sozialität \& Identität in synchroner computervermittelter Kommunikation. Stuttgart: Ibidem. S. 67-401.

Androutsopoulos, Jannis (2001):Von fett zu fabelhaft: Jugendsprache in der Sprachbiographie. In: Gessinger, Joachim/Sachweh, Svwenja (Hg.): Sprechalter. Osnabrücker Beiträge zur Sprachtheorie 62, S. 55-78. 
Androutsopoulos, Jannis (2001): Textsorten und Fankulturen. In: Klein, Josef/ Habscheid, Stephan/Fix, Ulla (Hg.): Kulturspezifik von Textsorten. Tübingen: Stauffenburg. S. 33-50.

Androutsopoulos, Jannis (2001): selbst, wenn es in der Bravo steht: Medien über Medien in der Jugendkultur. In: merz [medien + erziehung] 2/ 2001, S. 86-94.

Androutsopoulos, Jannis (2001): What names reveal about the music style: A study of naming patterns in popular music. In: Németh, Enikö (Hg.): Pragmatics in 2000. Antwerp: International Pragmatics Association. Vol. 2, S. 16-29.

Iordanidou, Anna/Androutsopoulos, Jannis (2001): Youth slang in Modern Greek. In: Georgakopoulou, Alexandra/Spanaki, Marianna (Hg.): A Reader in Greek Sociolinguistics. Frankfurt a. M.: Peter Lang. S. 285-302. Androutsopoulos, Jannis (2001) [in griechischer Sprache]: Linguistic approaches to journalistic language: genres, variation and ideology. In: Journalists' Union of the Athens Daily Newspapers (Hg.): Journalism and language. Athens: ESIEA. S. 167-184.

Androutsopoulos, Jannis (2001): Kontaktanzeigen - und was man damit machen kann. In: Der Deutschunterricht 2/2001, S. 88-91.

Androutsopoulos, Jannis (2001): Rezension „Eva Martha Eckkrammer/ Eder, Maria Hildegund: (Cyber)Diskurs zwischen Konvention und Revolution. Frankfurt. 2000. 344 S.“ In: sprache@web, Universität Hannover. http://www.websprache.net/literatur/rezensionen/3631348312.htm

\section{Dr. Karl-Heinz Bausch}

Bausch, Karl-Heinz/Steffens, Doris (2001): „Deutsche und Bulgaren im Gespräch“, Universität Sofia. In: Sprachreport 3/2001, S. 24.

\section{Cyril Belica}

Belica, Cyril (2001): Von absoluten Häufigkeiten zum Differenzenkoeffizienten. http://www.ids-mannheim.de/kt/diffcoeff.doc

\section{PD Dr. Nina Berend}

Knipf-Komlósi, Elisabeth/Berend, Nina (Hg.) (2001): Regionale Standards. Sprachvariationen in deutschsprachigen Ländern. Budapest-Pécs: Dialóg Campus Kiadó. S. 164.

\section{PD Dr. Hardarik Blühdorn}

Blühdorn, Hardarik (2001): Generische Referenz: Ein semantisches oder ein pragmatisches Phänomen? In: Deutsche Sprache 29, S. 1-19.

Blühdorn, Hardarik (2001): A codificação de informação espacial no alemão e no português do Brasil: Adposições e advérbios como meios para especificar relações estáticas [Die Kodierung von Rauminformation im Deutschen und im brasilianischen Portugiesisch: Adpositionen und Adverbien als Mittel zur Spezifizierung statischer Relationen]. São Paulo: Humanitas Publicações. $400 \mathrm{~S}$. 


\section{Michael Beißwenger}

Beißwenger, Michael (2001): Stoibers Kreuzzug und der Canossa-Gang des Bundestrainers. Spuren von Geschichtlichem in metaphorischen Wendungen der Alltagssprache. In: Lehr, Andrea/Kammerer, Matthias et al. (Hg.): Sprache im Alltag. Beiträge zu neuen Perspektiven in der Linguistik. Herbert Ernst Wiegand zum 65. Geburtstag gewidmet. Berlin u. a.: de Gruyter. S. 129-149.

Beißwenger, Michael (Hg.) (2001): Chat-Kommunikation. Sprache, Interaktion, Sozialität \& Identität in synchroner computervermittelter Kommunikation. Perspektiven auf ein interdisziplinäres Forschungsfeld. Stuttgart: Ibidem-Verlag. $580 \mathrm{~S}$.

Beißwenger, Michael (2001): Einleitung. In: Beißwenger, Michael (Hg.): Chat-Kommunikation. Sprache, Interaktion, Sozialität \& Identität in synchroner computervermittelter Kommunikation. Perspektiven auf ein interdisziplinäres Forschungsfeld. Stuttgart: Ibidem-Verlag. S. IX-XVI.

Beißwenger, Michael (2001): Introduction. In: Beißwenger, Michael (Hg.): Chat-Kommunikation. Sprache, Interaktion, Sozialität \& Identität in synchroner computervermittelter Kommunikation. Perspektiven auf ein interdisziplinäres Forschungsfeld. Stuttgart: Ibidem-Verlag. S. XVII-XXIII.

Beißwenger, Michael (2001): Das interaktive Lesespiel. Chat-Kommunikation als mediale Inszenierung. In: Beißwenger, Michael ( $\mathrm{Hg}$.): ChatKommunikation. Sprache, Interaktion, Sozialität \& Identität in synchroner computervermittelter Kommunikation. Perspektiven auf ein interdisziplinäres Forschungsfeld. Stuttgart: Ibidem-Verlag. S. 79-138.

Beißwenger, Michael/Pütz, Ulrike (2001): ,hauptsache wir verstehen uns gegeneinander". Ver(fremd)sprachlichung und Ausdrucksbildung in textbasierten Gehörlosen-Webchats. In: Beißwenger, Michael (Hg.): ChatKommunikation. Sprache, Interaktion, Sozialität \& Identität in synchroner computervermittelter Kommunikation. Perspektiven auf ein interdisziplinäres Forschungsfeld. Stuttgart: Ibidem-Verlag. S. 403-429.

Beißwenger, Michael (2001): Rezension: „Hans-Rainer Beck: Politische Rede als Interaktionsgefüge: Der Fall Hitler. Tübingen: Niemeyer. 2001. 225 S. (Linguistische Arbeiten 436)“. In: Zeitschrift für Angewandte Linguistik 35, S. 105-118.

\section{Dr. Eva Breindl}

Breindl, Eva (2001): Rezension: „Pittner, Karin: Adverbiale im Deutschen. Untersuchungen zu ihrer Stellung und Interpretation. Tübingen: Stauffenburg Verlag 1999“. In: Zeitschrift für Sprachwissenchaft 20/2, S. $280-286$.

Breindl, Eva (2001): Präpositionalphrasen. http://hypermedia.ids-mannheim.de/grammis/ 


\section{Dr. Elke Donalies}

Donalies, Elke (2000): Wortbildungspflege. Folge 3: Azubi mit Abi. In: Sprachreport 4/2000, S. 30. (Nachtrag).

Donalies, Elke (2001): Wortbildungspflege. Folge 4: Der maskulistische Hexerich. In: Sprachreport 1/2001, S. 24-26.

Donalies, Elke (2001): Wortbildungspflege. Folge 5: Über Liebes und Böses. In: Sprachreport 2/2001, S. 29.

Donalies, Elke (2001): Wortbildungspflege. Folge 6: Donaudampfschifffahrtskapitänskompositabildungsexpertenrunde. In: Sprachreport 3/2001, S. $17 \mathrm{f}$.

Donalies, Elke (2001): Wortbildungspflege. Folge 7: Hippes Sterbing. In: Sprachreport 4/2001, S. 25 f.

\section{Dr. Mechthild Elstermann}

Elstermann, Mechthild (2001): Rezension: Die Sprachmauer. In: Sprachreport $3 / 2001$, S. 5-8.

\section{Prof. Dr. Reinhard Fiehler}

Fiehler, Reinhard (2000): Gesprochene Sprache - gibt's die? In: Ágel, V./ Herzog, A. (Hg.): Jahrbuch der ungarischen Germanistik 2000. Budapest/ Bonn. S. 93-104. (Nachtrag).

Fiehler, Reinhard (2001): Die kommunikative Verfertigung von Altersidentität. In: Sichelschmidt, Lorenz/Strohner, Hans (Hg.): Sprache, Sinn und Situation. Festschrift für Gert Rickheit zum 60. Geburtstag. Wiesbaden: Deutscher Universitäts-Verlag. S. 125-144.

Fiehler, Reinhard (2001): Emotionalität im Gespräch. In: Brinker, Klaus/ Antos, Gerd/Heinemann, Wolfgang/Sager, Sven F. (Hg.): Text- und Gesprächslinguistik. Ein internationales Handbuch zeitgenössischer Forschung. 2. Halbband: Gesprächslinguistik. Berlin/New York: de Gruyter. S. 1425-1438. (Handbücher zur Sprach- und Kommunikationswissenschaft 16.2).

Fiehler, Reinhard (2001): Gesprächsanalyse und Kommunikationstraining. In: Brinker, Klaus/Antos, Gerd/Heinemann, Wolfgang/Sager, Sven F. (Hg.): Text- und Gesprächslinguistik. Ein internationales Handbuch zeitgenössischer Forschung. 2. Halbband: Gesprächslinguistik. Berlin/ New York: de Gruyter. S. 1697-1710. (Handbücher zur Sprach- und Kommunikationswissenschaft 16.2).

\section{Dr. Kristine Fischer-Hupe}

Fischer-Hupe, Kristine (2001): Zur Entstehungs-, Editions- und Rezeptionsgeschichte von Victor Klemperers „LTI“. In: Siehr, Karl-Heinz (Hg.): Victor von Klemperers Werk. Texte und Materialien für Lehrer. Berlin: Aufbau Taschenbuch Verlag. S. 32-53.

Fischer-Hupe, Kristine (2001): Zu Victor Klemperers „LTI“: ein Auswahl- 
register für den Schulgebrauch. In: Siehr, Karl-Heinz (Hg.): Victor von Klemperers Werk. Texte und Materialien für Lehrer. Berlin: Aufbau Taschenbuch Verlag. S. 291-301.

\section{Prof. Dr. Claudia Fraas}

Fraas, Claudia (2001): Usuelle Wortverbindungen als sprachliche Manifestation von Bedeutungswissen. Theoretische Begründung, Methodischer Ansatz und empirische Befunde. In: Nikula, Henrik/Drescher, Robert (Hg.): Lexikon und Text. Vaasa. S. 41-66.

\section{Daniel Glatz}

Glatz, Daniel (2001): Zur Ereignisstruktur von Kommunikationsverben. In: Harras, Gisela (Hg.): Kommunikationsverben. Konzeptuelle Ordnung und semantische Repräsentation. Tübingen: Narr. S. 33-59. (Studien zur deutschen Sprache Bd. 24).

\section{Prof. Dr. Gisela Harras}

Harras, Gisela (2001): Alltag, Lebenswelt, Lebensform und Sprache. Ein Gespräch. In: Lehr, Andrea/Kammerer, Matthias et alii (Hg.): Sprache im Alltag. Beiträge zu neuen Perspektiven in der Linguistik. Herbert Ernst Wiegand zum 65. Geburtstag gewidmet. Berlin/New York. S. 41-55.

Harras, Gisela (Hg.) (2001): Kommunikationsverben: Konzeptuelle Ordnung und semantische Repräsentation. Tübingen: Narr. 229 S. (Studien zur deutschen Sprache Bd. 24).

Harras, Gisela (2001): Performativität, Sprechakte und Sprechaktverben. In: Harras, Gisela (Hg.): Kommunikationsverben: Konzeptuelle Ordnung und semantische Repräsentation. Tübingen: Narr. S. 11-32.

Harras, Gisela (2001): Synonymie und Synonymik. In: Harras, Gisela (Hg.): Kommunikationsverben: Konzeptuelle Ordnung und semantische Repräsentation. Tübingen: Narr. S. 131-193.

\section{Prof. Dr. Ulrike Haß-Zumkehr}

Haß-Zumkehr, Ulrike (2001): Deutsche Wörterbücher - Brennpunkt von Sprach- und Kulturgeschichte, Berlin: de Gruyter. 411 S.

Haß-Zumkehr, Ulrike (2001): Zur Mikrostruktur im Hypertext-Wörterbuch. In: Lemberg, Ingrid/Schröder, Bernhard/Storrer, Angelika (Hg.): Chancen und Perspektiven computerunterstützter Lexikographie. Tübingen: Niemeyer. S. 103-115 (Lexicographica. Series Maior, 107).

Haß-Zumkehr, Ulrike (2001): Sprache im Alltag als Konstruktion von Lexikografie und Sprachwissenschaft. In: Lehr, Andrea/Kammerer, Matthias/Konerding, Klaus-Peter/Storrer, Angelika/Thimm, Caja/Wolski, Werner (Hg.): Sprache im Alltag. Beiträge zu neuen Perspektiven in der Linguistik. Herbert Ernst Wiegand zum 65. Geburtstag gewidmet. Berlin. S. $57-70$. 
Haß-Zumkehr, Ulrike (2001): Hermann Paul. In: Neue Deutsche Biographie. Hrsg. v. d. Historischen Kommission bei der Bayerischen Akademie der Wissenschaften. 20. Bd., Berlin. S. 115-116.

Haß-Zumkehr, Ulrike (2001): Die Grenzen des Stolzes im Deutschen. In: Sprachreport 2/2001, S. 2-4.

Haß-Zumkehr, Ulrike (2001): Agathe Lasch (1879-1942?). In: Barner, Wilfried/König, Christoph (Hg.): Jüdische Intellektuelle und die Philologien in Deutschland 1871-1933. Göttingen: Wallstein. S. 203-211. (Marbacher Wissenschaftsgeschichte Bd. 3).

Haß-Zumkehr, Ulrike (2001): Zur Sprachkritik der Achtundsechziger. In: Ott, Ulrich/Luckscheiter, Roman (Hg.): Belles lettres/Graffiti. Soziale Phantasien und Ausdrucksformen der Achtundsechziger. Göttingen: Wallstein. S. 115-121.

\section{Dr. Klaus Heller}

Heller, Klaus (2001): Statement zu „Verändert elektronische Kommunikation unsere Sprache?" In: New World 1/2001, S. 7.

Heller, Klaus (2001): Rechtschreibung 2000. Wörterliste der geänderten Schreibungen. Lizenzausgabe in bulgarischer Sprache. Sofia. $78 \mathrm{~S}$. Heller, Klaus (2001): Die Last der Freiheit oder Von den engen Grenzeneiner weit gedachten Norm. Erfahrungen bei der Neuregelung der deutschen Rechtschreibung. In: Text und Kontext. Zeitschrift für germanistische Literaturforschung in Skandinavien. Kopenhagen/München, Jg. 23, Heft 2, S. 311-331. (Nachdruck aus Språkbrukeren - fri til å velge? Artikler om homogen og heterogen språknorm. Kristiansand 1999).

\section{Prof. Dr. Dieter Herberg}

Herberg, Dieter (2001): Neologismen der Neunzigerjahre. In: Stickel, Gerhard (Hg.): Neues und Fremdes im deutschen Wortschatz. Aktueller lexikalischer Wandel. Berlin/New York. S. 89-104. (Jahrbücher des Instituts für deutsche Sprache).

Herberg, Dieter (2001): Anglizismen: Kein Grund zur Panik. Unverdauliche Wörter werden wieder abgestoßen. In: Journalistik Journal, Jg. 4, Nr. 1, S. $22 \mathrm{f}$.

Herberg, Dieter (2001): Euro: The Career of a European Neologism in German Press Texts (1995-1999). In: Musolff, Andreas/Good, Colin/Points, Petra/Wittlinger, Ruth (Hg.): Attitudes Towards Europe. Language in the unification process. Aldershot/Burlington USA/Singapore/Sydney: Ashgate Publishing Ltd. S. 113-151.

Herberg, Dieter (2001): Euro. Zur Karriere eines europäischen Neologismus in deutschen Pressetexten (1995-1999). http://www.dur.ac.uk/SMEL/ depts/german/eurometa/Eurocorp-analysis.htm Herberg, Dieter/Steffens, Doris/Tellenbach, Elke (2001): Schlüsselwörter der Wendezeit. Wörter-Buch zum öffentlichen Sprachgebrauch 1989/90. 
Berlin, New York: Walter de Gruyter 1997. (Schriften des Instituts für Deutsche Sprache 6). (CD-Rom Sofia 2001).

Herberg, Dieter (2001): Plädoyer für mehr Sensibilität beim Übereinanderreden. In: Antos, Gerd/Fix, Ulla/Kühn, Ingrid (Hg.): Deutsche Sprachund Kommunikationserfahrungen zehn Jahre nach der „Wende“. Frankfurt am Main/Berlin/Bern. S. 261-263. (Wittenberger Beiträge zur deutschen Sprache und Kultur 2).

\section{Prof. Dr. Werner Kallmeyer}

Kallmeyer, Werner/Streeck, Jürgen (2001): Interaction by inscription. In: Journal of Pragmatics 33, S. 465-490.

Kallmeyer, Werner (2001): Beraten und Betreuen. Zur gesprächsanalytischen Untersuchung von helfenden Interaktionen. In: Zeitschrift für Qualitative Bildungs-, Beratungs- und Sozialforschung 2, S. 227-252.

Kallmeyer, Werner (2001): Perspektivenumkehrung als Element des emanzipatorischen Stils in Migrantengruppen. In: Jakobs, Eva Maria/ Rothkegel, Annelie (Hg.): Perspektiven auf Stil. Festschrift für Barbara Sandig zum 60. Geburtstag. Tübingen. S. 397-418.

\section{Dr. Heidrun Kämper}

Kämper, Heidrun (2001): Words, Phrases and Argumentational Structures in the German Debate on Europe in the Early Post-War Period. In: Musolff, Andreas/Good, Colin/Points, Petra/Wittlinger, Ruth (Hg.): Attitudes Towards Europe. Language in the unification process. Aldershot/Burlington USA/Singapore/Sydney: Ashgate Publishing Ltd. S. 89-113.

Kämper, Heidrun (2001): Europa - Formeln, Begriffe und Argumente in der frühen Nachkriegszeit. http://www.dur.ac.uk/SMEL/depts/german/eurometa/EuroIdee-analysis.htm

Kämper, Heidrun (2001): Die Amerikanisierung der deutschen Sprache. In: Junker, Detlef/Gassert, Philipp/Mausbach, Wilfried/Morris, David B. (Hg.): Die USA und Deutschland im Zeitalter des Kalten Krieges 1945-1990. Ein Handbuch. Stuttgart/München: Deutsche Verlags-Anstalt. S. $496-507$.

Kämper, Heidrun (2001): Das Sprach- und Kulturkonzept Victor Klemperers. In: Siehr, Karl-Heinz (Hg.): Victor von Klemperers Werk. Texte und Materialien für Lehrer. Berlin: Aufbau Taschenbuch Verlag. S. 53-69.

Kämper, Heidrun (2001): Jugendsprache um 1900 und die schöne Literatur. In: Der Deutschunterricht 1/2001, S. 47-58.

\section{PD Dr. Inken Keim}

Keim, Inken (2000): Der Umgang mit territorialen Ansprüchen als Merkmal sozialen Stils. Am Beispiel von Interaktionen aus zwei Frauengruppen in Mannheim. In: Lüger, Heinz-Helmut (Hg.): Höflichkeitsstile. Berlin/New York. S. 187-212. (Nachtrag).

Keim, Inken (2001): Klatsch und Tratsch als lustvolles Gruppenerlebnis. Eine ethnographisch-soziolinguistische Studie. In: Ivanyi, Zsuzsan- 
na (Hg.): Gesprächsforschung: Tendenzen und Perspektiven. Debrecen. S. 131-153.

\section{Dr. Michael Kinne}

Kinne, Michael (2001): Aus dem Unwörterbuch des Menschen. Über ein Lexikon mit verletzenden, verharmlosenden, täuschungsvollen und beschönigenden, aber auch mit ziemlich harmlosen und sehr vielen ganz braven Wörtern (Rezension). In: Sprachreport 1/2001, S. 18-19.

\section{Ralf Knöbl}

Knöbl, Ralf (2001): Form und Funktion von Sprachmischung. Erstes Kolloquium der DFG-Forschergruppe ,Sprachvariation als kommunikative Praxis“ (Mannheim, 1.-2. Dezember 2000). In: Deutsche Sprache 1/2001, S. $90-96$.

\section{Dr. Anja Lobenstein-Reichmann}

Lobenstein-Reichmann, Anja (2001): Vom Adel christlicher Freiheit. In: Hartweg, Frédéric (Hg.): Martin Luther. Strasbourg. S. 63-137. Lobenstein-Reichmann, Anja (2001): Rezension: „Rudolf Grosse (Hg.): Bedeutungserfassung und Bedeutungsbeschreibung in historischen und dialektologischen Wörterbüchern. Stuttgart, Leipzig: Hirzel. 1998“. In: Donhauser, Karin et alii. (Hg.): Beiträge zur Geschichte der Deutschen Sprache und Literatur. Bd. 123, Heft 1, S. 99-103.

Lobenstein-Reichmann, Anja/Reichmann, Oskar (2001): „... iederman wolt gen himl". Das frühneuhochdeutsche Wörterbuch als Spiegel der Kulturgeschichte. In: Der Sprachdienst, Jahrgang 45, Heft 4, S. 134-141. Anderson, Robert R./Goebel, Ulrich/Reichmann, Oskar (Hg.): Frühneuhochdeutsches Wörterbuch. Berlin/New York: de Gruyter. $1989 \mathrm{ff}$. hier: Frühneuhochdeutsches Wörterbuch (2001): gnacke - grossprecher. Bearbeitet von Anja Lobenstein-Reichmann. Berlin/New York: de Gruyter. 2001. Spalten 1-512. (Frühneuhochdeutsches Wörterbuch 7/Lieferung 1).

\section{Prof. Dr. Katharina Meng}

Meng, Katharina (2001): Russlanddeutsche Sprachbiografien. Untersuchungen zur sprachlichen Integration von Aussiedlerfamilien. Unter Mitarbeit von Ekaterina Protassova. Tübingen: Gunter Narr. 549 S. (Studien zur deutschen Sprache Bd. 21).

Meng, Katharina (2001): Sprachliche Integration von Aussiedlern. Einige Ergebnisse, einige Probleme. In: Sprachreport 2/2001, S. 4-11.

\section{PD Dr. Gereon Müller}

Müller, Gereon (2000): Elemente der optimalitätstheoretischen Syntax. Tübingen: Stauffenburg Verlag. 344 S. (Erschienen 2001).

Müller, Gereon/Sternefeld, Wolfgang (Hg.) (2001): Competition in Syntax. Berlin: Mouton de Gruyter. $410 \mathrm{~S}$.

Müller, Gereon/Sternefeld, Wolfgang (2001): The Rise of Competition in 
Syntax: A Synopsis. In: Müller, Gereon/Sternefeld, Wolfgang (Hg.): Competition in Syntax. Berlin: Mouton de Gruyter. S. 1-68.

Müller, Gereon (2001): Order Preservation, Parallel Movement, and the Emergence of the Unmarked. In: Legendre, Géraldine/Grimshaw, Jane/ Vikner, Sten (Hg.): Optimality-Theoretic Syntax. Cambridge, Mass: MIT Press. S. 279-313.

\section{Kristel Proost}

Proost, Kristel (2001): Zum Lexikalisierungsraum kommunikativer Konzepte. In: Harras, Gisela (Hg.) (2001): Kommunikationsverben: Konzeptuelle Ordnung und semantische Repräsentation. Tübingen: Narr. S. 77-129. (Studien zur deutschen Sprache Bd. 24).

Proost, Kristel (2001): Warum LÜGEN \& CO., aber nicht WAHRSAGEN? Asymmetrien im Wortschatz. In: Sprachreport 1/2001. S. 14-17.

\section{Dr. Herbert Schmidt}

Schmidt, Herbert (Hg.) (2001): Einführung in die Diachrone Sprachwissenschaft. Ein Lehr-, Studien- und Übungsbuch für Germanisten. 5., verbesserte, erweiterte und vollständig überarbeitete Auflage. Göppingen. 293 S. (Göppinger Arbeiten zur Germanistik; 459).

\section{Dr. Rudolf Schmidt}

Schmidt, Rudolf (2001): Instrumente zur Erstellung multimedialer Gesprächskorpora. In: Lobin, H. (Hg.): Sprach- und Texttechnologie in digitalen Medien - Proceedings der GLDV-Frühjahrstagung 2001. Norderstedt. S. 115-127.

\section{Dr. Wilfried Schütte}

Schütte, Wilfried/Born, Joachim (2000): Die Stellung des Deutschen in den europäischen Institutionen. In: Besch, Werner/Betten, Anne/Reichmann, Oskar/Sonderegger, Stefan (Hg.): Sprachgeschichte. Ein Handbuch zur Geschichte der deutschen Sprache und ihrer Erforschung. 2. Auflage. Berlin/New York. S. 2175-2186.

Schütte, Wilfried (2001): Alltagsgespräche. In: Brinker, Klaus/Antos, Gerd/Heinemann, Wolfgang/Sager, Sven F.(Hg.): Text- und Gesprächslinguistik. Linguistics of Text and Conversation. Ein internationales Handbuch zeitgenössischer Forschung. An International Handbook of Contemporay Research. 2. Halbband. Berlin/New York: de Gruyter. S. 1485-1492.

\section{Dr. des Ulrich Schnörch}

Schnörch, Ulrich (2001): PONS Großes Schulwörterbuch Deutsch. Stuttgart/Düsseldorf/Leipzig: Ernst Klett Verlag. $1380 \mathrm{~S}$. (Ulrich Schnörch = Mitautor).

Schnörch, Ulrich (2001): Lemmatisierung und Wortschatzauswahl. http:/ /www.ids-mannheim.de/wiw/lemmatisierung.html

Schnörch, Ulrich (2001): Deskriptive vs. Präskriptive Ausrichtung - 
Sprachreflexion. http://www.ids-mannheim.de/wiw/sprachreflexion.html Schnörch, Ulrich (2001): Historische und sachliche Zusatzinformationen. http://www.ids-mannheim.de/wiw/gesa.html

\section{PD Dr. Thomas Spranz-Fogasy}

Spranz-Fogasy, Thomas (2001): Zur Methodologie der Handlungsstrukturanalyse von Gesprächen (mit Carmen Spiegel). In: Ivanyi, S. (Hg.): Gesprächsforschung. Reihe Metalinguistica. Frankfurt. S. 243-257.

Spranz-Fogasy, Thomas/Deppermann, A. (2001): Teilnehmende Beobachtung in der Gesprächsanalyse. In: Brinker, K./Antos, G./Heinemann, W./ Sager, S.F. (Hg.): Text- und Gesprächslinguistik. Handbücher zur Sprachund Kommunikationswissenschaft. Berlin/New York. S. 1007-1013.

Spranz-Fogasy, Thomas/Deppermann, A. (2001): Aspekte und Merkmale der Gesprächssituation. In: Brinker, K./Antos, G./Heinemann, W./Sager, S.F. (Hg.). Text- und Gesprächslinguistik. Handbücher zur Sprach- und Kommunikationswissenschaft. Berlin/New York. S. 1148-1161.

Spranz-Fogasy, Thomas/Spiegel, C. (2001): Aufbau und Abfolge von Gesprächsphasen. In: Brinker, K./Antos, G./Heinemann, W./Sager, S.F. (Hg.): Text- und Gesprächslinguistik. Handbücher zur Sprach- und Kommunikationswissenschaft. Berlin/New York. S. 1241-1252.

\section{Dr. Doris Steffens}

Bausch, Karl-Heinz/Steffens, Doris (2001): „Deutsche und Bulgaren im Gespräch“, Universität Sofia. In: Sprachreport 3/2001, S. 24.

Herberg, Dieter/Steffens, Doris/Tellenbach, Elke (2001): Schlüsselwörter der Wendezeit. Wörter-Buch zum öffentlichen Sprachgebrauch 1989/90. Berlin, New York: Walter de Gruyter 1997. (Schriften des Instituts für Deutsche Sprache 6). (CD-Rom Sofia 2001).

\section{Dr. Kathrin Steyer}

Steyer, Kathrin (2001): Usuelle Wortverbindungen. http:/www/wiw/ mehrwort.shtml

Steyer, Kathrin (2001): Pragmatische Perspektive. http://www/wiw/pragmatik.html

\section{Prof. Dr. Gerhard Stickel}

Stickel, Gerhard (2001): Wissenschaftssprachen an deutschen Forschungsinstituten. In: Ehlich, Konrad/Ossner, Jakob/Stammerjohann, Harro (Hg.): Hochsprachen in Europa. Entstehung, Geltung, Zukunft. Freiburg i.Br. S. 309-320.

Stickel, Gerhard (2001): Deutsch als Wissenschaftssprache an außeruniversitären Forschungsinstituten. In: Debus, Friedhelm/Krollmann, Franz Gustav/Pörksen, Uwe (Hg.): Deutsch als Wissenschaftssprache im 20. Jahrhundert. Vorträge des Internationalen Symposions vom 18./19. Januar 2000. Mainz: Akademie der Wiss. und der Literatur. Stuttgart: Steiner. S. $125-142$. 
Stickel, Gerhard/Gerhardt, Rudolf (2001): Die Sprache der Juristen ist besser als ihr Ruf. In: Zeitschrift für Rechtspolitik mit Rechtspolitischer Umschau, Mai 2001, 34. Jg., S. 229-232.

Stickel, Gerhard (2001): Memorandum: Politik für die deutsche Sprache. In: Sprachreport 3/2001, S. 8-11.

Stickel, Gerhard (2001): Ost- und westdeutsche Spracheinstellungen. In: Kühn, Ingrid (Hg.): Ost-West-Sprachgebrauch - zehn Jahre nach der Wende. Eine Disputation. Opladen. S. 51-64.

Stickel, Gerhard (2001): (Hg.): Neues und Fremdes im deutschen Wortschatz. Aktueller lexikalischer Wandel. Jahrbuch 2000 des Instituts für Deutsche Sprache. Berlin/New York. VIII, 388 S.

\section{Dr. Angelika Storrer}

Storrer, Angelika (2001): Sprachliche Besonderheiten getippter Gespräche: Sprecherwechsel und sprachliches Zeigen in der Chat-Kommunikation. In: Beißwenger, Michael (Hg.): Chat-Kommunikation. Sprache, Interaktion, Sozialität und Identität in synchroner computervermittelter Kommunikation. Stuttgart: ibidem. S. 3-24.

Lehr, Andrea/Kammerer, Matthias/Konerding, Klaus-Peter/Storrer, Angelika/Thimm, Caja/Wolski, Werner (Hg.) (2001): Sprache im Alltag. Beiträge zu neuen Perspektiven in der Linguistik. Herbert Ernst Wiegand zum 65. Geburtstag gewidmet. Berlin u. a.: de Gruyter. S. 634.

Storrer, Angelika (2001): Getippte Gespräche oder dialogische Texte? Zur kommunikationstheoretischen Einordnung der Chat-Kommunikation. In: Lehr, Andrea/Kammerer, Matthias et al. (Hg.): Sprache im Alltag. Beiträge zu neuen Perspektiven in der Linguistik. Herbert Ernst Wiegand zum 65. Geburtstag gewidmet. Berlin u. a.: de Gruyter. S. 439-465.

Storrer, Angelika (2001): Schreiben, um besucht zu werden: Textgestaltung fürs World Wide Web. In: Bucher, Hans-Jürgen/Püschel, Ulrich (Hg.): Die Zeitung zwischen Print und Digitalisierung. Opladen/Wiesbaden: Westdeutscher Verlag. S. 173-205.

Storrer, Angelika (2001): Neue Medien - neue Stilfragen: Das World Wide Web unter stilistischer Perspektive. In: Jakobs, Eva-Maria/Rothkegel, Annely (Hg.): Perspektiven auf Stil. Tübingen: Niemeyer. S. 101-124.

Lemberg, Ingrid/Schröder, Bernhard/Storrer, Angelika (Hg.) (2001): Chancen und Perspektiven computergestützter Lexikographie. Hypertext, Internet und SGML/XML für die Produktion und Publikation digitaler Wörterbücher. Tübingen: Niemeyer. $270 \mathrm{~S}$.

Lemberg, Ingrid/Schröder, Bernhard/Storrer, Angelika: Einführung. In: Lemberg, Ingrid/Schröder, Bernhard/Storrer, Angelika (Hg.) (2001): Chancen und Perspektiven computergestützter Lexikographie. Hypertext, Internet und SGML/XML für die Produktion und Publikation digitaler Wörterbücher. Tübingen: Niemeyer. S. 1-4. 
Storrer, Angelika (2001): Digitale Wörterbücher als Hypertexte: Zur Nutzung des Hypertextkonzepts in der Lexikographie. In: Lemberg, Ingrid/ Schröder, Bernhard/Storrer, Angelika (Hg.): Chancen und Perspektiven computergestützter Lexikographie. Hypertext, Internet und SGML/XML für die Produktion und Publikation digitaler Wörterbücher. Tübingen: Niemeyer. S. 88-104.

Cölfen, Hermann/Liebert, Wolf-Andreas/Storrer, Angelika (Hg.) (2001): Wissenskonstruktion mit Hypermedien. Osnabrücker Beiträge zur Sprachtheorie. OBST 63/2001. $164 \mathrm{~S}$.

\section{Prof. Dr. Bruno Strecker}

Strecker, Bruno (2000): Logik und die Bedeutung von Sätzen der deutschen Sprache. In: Lueken, Geert-Lueke (Hg.): Formen der Argumentation, Leipzig. S. 131-140. (Nachtrag).

Strecker, Bruno (2001): Grammatik in Forschung und Unterricht. In: Grammatik und Grammatikunterricht. Mitteilungen des Deutschen Germanistenverbands, Heft 1/2001, S. 10-17.

Strecker, Bruno (2001): Kurze Präsentation der „Grammatik der deutschen Sprache" mit einem Ausblick auf neuere Formen der Grammatikschreibung. In: Grammatik und Grammatikunterricht. Mitteilungen des Deutschen Germanistenverbands, Heft 1/2001, S. 108-117.

\section{Dr. Elke Tellenbach}

Herberg, Dieter/Steffens, Doris/Tellenbach, Elke (2001): Schlüsselwörter der Wendezeit. Wörter-Buch zum öffentlichen Sprachgebrauch 1989/90. Berlin, New York: Walter de Gruyter 1997. (Schriften des Instituts für Deutsche Sprache 6). (CD-Rom Sofia 2001).

Tellenbach, Elke (2001): Neologismen der neunziger Jahre. Vom Textkorpus zur Datenbank. In: Barz, Irmhild/Fix, Ulla/Lerchner, Gotthard (Hg.): Das Wort in Text und Wörterbuch. Leipzig. S. 105-118. (Abhandlungen der Sächsischen Akademie der Wissenschaften zu Leipzig. Philologischhistorische Klasse. Band 76, Heft 4).

\section{Dr. Annette Trabold}

Trabold, Annette (2001): Deutsch - eine Sprache, die Menschen und Kulturen näher bringt. In: Sprachreport 3/2001, S. 23.

\section{Dr. Ulrich Hermann Waßner}

Waßner, Ulrich Hermann (Hg.) (2001): Lingua et Linguae. Festschrift für Clemens-Peter Herbermann zum 60. Geburtstag. Aachen: Shaker Verlag. VIII/471 S. (Bochumer Beiträge zur Semiotik. Neue Folge 6.).

Waßner, Ulrich Hermann (2001): Halb zog sie ihn, halb sank er hin - Anmerkungen zu einem phraseologischen Konnektor des Deutschen. In: Waßner, Ulrich Hermann (Hg.), Lingua et Linguae. Festschrift für Cle- 
mens-Peter Herbermann zum 60. Geburtstag. Aachen: Shaker Verlag. S. 447-468. (Bochumer Beiträge zur Semiotik. Neue Folge 6).

\section{Dr. Edeltraud Winkler}

Winkler, Edeltraud (2001): Möglichkeiten der semantischen Dekomposition von Kommunikationsverben. In: Harras, Gisela (Hg.): Kommunikationsverben. Konzeptuelle Ordnung und semantische Repräsentation. Tübingen: Narr. S. 61-76. (Studien zur deutschen Sprache Bd. 24).

Winkler, Edeltraud (2001): Aufbau und Gliederung einer Synonymik deutscher Sprechaktverben. In: Harras, Gisela (Hg.): Kommunikationsverben. Konzeptuelle Ordnung und semantische Repräsentation. Tübingen: Narr. S. 61-76. (Studien zur deutschen Sprache Bd. 24).

\section{Prof. Dr. Gisela Zifonun}

Zifonun, Gisela (2000):,,Man lebt nur einmal“". Morphosyntax und Semantik des Pronomens man. In: Deutsche Sprache 3/2000, S. 232-253. (Nachtrag).

Zifonun, Gisela (2001): Die „Grammatik der deutschen Sprache“: eine wissenschaftliche Grammatik als Schlüssel zu authentischem Gegenwartsdeutsch. In: Zickfeldt, Aug. Wilhelm/Issel, Burkhard/Ehlich, Konrad (Hg.): Deutsch in Norwegen. Neue Beiträge im Gespräch zwischen Germanistik, Lehrerausbildung und Schule. Regensburg. S. 40-58. (Materialien Deutsch als Fremdsprache 62).

Zifonun, Gisela (2001): Eigennamen in der Narrenschlacht. Oder: Wie man Walther von der Vogelweide in den Genitiv setzt. In: Sprachreport 3/ 2001. S. 2-5.

Zifonun, Gisela (2001): Grammatik des Deutschen im europäischen Vergleich: Der Relativsatz. 104 S. (amades 3/01).

Zifonun, Gisela (2001): Grammatik des Deutschen im europäischen Vergleich: Das Pronomen Teil I. Überblick und Personalpronomen. (amades $4 / 01)$.

\section{Kontakte des IDS zu anderen Institutionen, Studien- aufenthalte und Besuche in- und ausländischer Wissenschaftler am IDS, Praktika, Besuchergruppen}

\subsection{Kontakte $\mathrm{zu}$ anderen Institutionen}

Accademia della Crusca, Florenz

Alexander-von-Humboldt-Stiftung, Bonn

Arbeitskreis der Sprachzentren, Sprachlehrinstitute und Fremdspracheninstitute

Arbeitsstelle Deutsches Wörterbuch, Berlin

Arbeitsstelle Deutsches Wörterbuch, Göttingen

Arbeitsstelle „Sprache in Südwestdeutschland“, Tübingen 
DANTE. Deutschsprachige Anwendervereinigung TEX e. V., Heidelberg

Deutsche Forschungsgemeinschaft (DFG), Bonn

Deutsche Gesellschaft für Sprachwissenschaft (DGfS)

Deutsche Gesellschaft für Dokumentation (DGD), Frankfurt a.M.

Deutscher Akademischer Austauschdienst (DAAD), Bonn

Deutscher Germanistenverband

Deutscher Sprachatlas, Marburg

DIN-Normenausschuss Terminologie, Berlin

Dudenredaktion des Bibliographischen Instituts, Mannheim

EURALEX, European Association for Lexicography, Exeter

Fachverband Deutsch als Fremdsprache

Fachverband Moderne Fremdsprachen

Forschungszentrum für die Landessprachen Finnlands, Helsinki

Fritz-Thyssen-Stiftung, Köln

GLDV, Gesellschaft für linguistische Datenverarbeitung, Frankfurt

Geisteswissenschaftliche Zentren, Berlin

Gesellschaft für angewandte Linguistik e. V. (GAL)

Gesellschaft für deutsche Sprache (GfdS), Wiesbaden

Gesellschaft für Mathematik und Datenverarbeitung $\mathrm{mbH}$, Bonn

Goethe-Institut/Inter Nationes, München

Hugo-Moser-Stiftung im Stifterverband für die Deutsche Wissenschaft, Essen

Institut für Auslandsbeziehungen, Stuttgart

International Association of Sound Archives (IASA)

Institut für niederdeutsche Sprache (INS), Bremen

Laboratoire d'Automatique Documentaire et Linguistique (LADL), Paris

Max-Planck-Institut für Psycholinguistik, Nijmegen

Nederlandse Taalunie, Den Haag

Nordischer Sprachenrat, Kopenhagen

Österreichische Akademie der Wissenschaften, Wien

Polnische Akademie der Wissenschaften, Warschau

Robert-Bosch-Stiftung, Stuttgart

Russische Akademie der Wissenschaften, Institut für russische Sprache, Moskau

Universitäten Mannheim und Heidelberg sowie zahlreiche germanistische Institute an weiteren Universitäten und Hochschulen im In- und Ausland Verein zur Förderung sprachwissenschaftlicher Studien, Berlin Volkswagen-Stiftung, Hannover

Wissenschaftsgemeinschaft Gottfried Wilhelm Leibniz (WGL) 
6.2 Studienaufenthalte und Besuche in- und ausländischer Wissenschaftler am IDS

Teuta Abrashi, M. A., Universität Prišstina, Kosovo, 19.7.-10.8.2001

Hasmik Adilkhanyan, Staatliche Universität Erevan, Armenien, 8.11.200126.1.2002

Margarita Aharonyan, Staatliche Universität Erevan, Armenien, 8.11.200126.1.2002

Mohammed al-Schaar, Universität Al-Minia, Ägypten, 1.9.2000-1.9.2005

Anna Arabyan, Staatliche Universität Erevan, Armenien, 8.11.200126.1.2002

Dr. Uldus Aslanowa, Universität Baku, Aserbaidschan, 17.9.-17.11.2001

Dr. Birte Asmuss, Universität Arhus, Dänemark, 23.4.-5.5.2001

Hrair Baghramyan, Staatliche Universität Erevan, Armenien, 17.11.200012.2.2001

Dr. Yasemin Balci, Marmara Universität, Istanbul, Türkei, 22.10.22.12.2001

Dr. Maria Teresa Bianco, Istituto Universitario Orientale, Napoli, Italien, 2.4.-28.5.2001

Edyta Blachut, M. A., Universität Breslau, Polen, 1.10.2001-31.7.2002

Thomas Blumenrath, Jerusan, Armenien, 8.1.-2.2.2001

Emilija Bojkovskr, M. A., Universität Skopje, Mazedonien, 20.2.-28.2.2001

Kalé Burridge, La Trobe Universität, Melbourne, Australien, 2.5.-1.6.2001

Prof. Dr. San-lii Chang, Fu-Jen Universität, Taipei, Taiwan, 20.6.-24.8.2001

Prof. Dr. Lesław Cirko, Universität Breslau, Polen, 17.7.-30.7.2001

Prof. Dr. Dr. h.c. Michael Clyne, Monash Universität, Melbourne, Australien, 12.6.-19.6.2001

Dr. Ruxandra Cosma, Universität Bukarest, Rumänien, 1.2.-30.6.2001

Dr. Winifred Davies, Universität Wales Aberystwyth, U.K., 1.11.30.11 .2001

Prof. Maria José Dominguez Vázquez, Universität Santiago de Compostela, Spanien, 1.7.-30.9.2001

Prof. Dr. Martin Durrell, Universität Manchester, Großbritannien, 1.10.1.11 .01

Prof. Dr. Csaba Földes, Universität Veszprém, Ungarn, 18.7.-25.7.2001

Prof. Dr. Jura Gabrieljan, Universität Erevan, Armenien, 8.1.-29.2.2001

Vahram Gharibjanyan, Universität Erevan, Armenien, 1.11.2000-15.2.2001

Prof. Wanheng Han $\dagger$, Tianjin, V. R. China, 24.10.2000-24.1.2001

Prof. Dr. Kenichi Hashimoto, Doshisha-Universität Kyoto, Japan, 12.4.2000-31.3.2002

Prof. Dr. Monica Heller, Universität Toronto, Ontario, Kanada, 10.9.31.12.2001

Karine Horhannisjan, Universität Erevan, Armenien, 1.12.2001-31.2.2002

Dr. Manshu Ide, Shinshu Universität Nagano, Japan, 7.11.2001-30.9.2002 
Dr. Uta Itakura, Keio-Universität, Tokio, Japan, 29.1.-31.3.2001

Gulnara Ishtuganova, Baschkirische Staatliche Universität Ufa, Russland, 14.8.2000-31.8.2002

Dr. Sally Johnson, Universität Lancaster, Großbritannien, 1.7.200030.6.2001

Prof. Dr. András Kertész, Universität Debrecen, Ungarn, 1.8.-31.10.2001

Olga Kortschigo, Leo-Tolstoi-Universität, Tula, Russland, 9.8.-27.9.2001

Dipl.germ. Jelena Kostić, Universität Belgrad, Jugoslawien, 1.7.31.8.2001

Dr. Olga Kostrowa, Pädagogische Universität, Samara, Russland, 23.10.24.10.2001

Prof. Dr. Elena Kubryakova, Linguistisches Institut Moskau, Russland Hung-Ling Kuo, Fu-Jen Universität Taipei, Taiwan, 20.6.-24.8.2001

Arusyak Kyurkchyan, Staatliche Universität Erevan, Armenien, 17.11.200012.2.2001

Prof. Dr. Duk Ho Lee, Universität Seoul, Korea, 22.6.-25.8.2001

Barbara Lübke, Universität Santiago de Compostela, Spanien, 5.7.-3.8.2001

Meike Meliss, Universität Santiago de Compostela, Spanien, 25.6.20.7.2001

Inga Meškauskiene, Pädagogische Universität Vilnius, Litauen, 8.1.8.3.2001

Hermine Nazaryan, Fremdsprachen-Hochschule Erevan, Armenien, 16.10.2000-1.3.2001

Klara Olah Töth, Universität Veszprém, Ungarn, 28.7.-25.7.2001

Aicha Ouaret, Universität Algier, Algerien, 17.10.-14.11.2001

Dr. Viktor Portiannikov, Universität Nishnij Novgorod, Russland, 17.10.19.10.2001

Beata Raczynska, Pädagogische Universität Vilnius, Litauen, 14.5.1.7.2001

Teresa Sanchez-Nieto, Universität Valladolid, Soria, Spanien, 3.7.30.7.2001

Dr. Alexander Sawjalow, Universität Nishnij Novgorod, Russland, 17.10.19.10.2001

Prof. Dr. Carlo Serra-Borneto, Universität La Sapienza, Rom, Italien, 21.5.25.5.2001

Swetlana Simonowa, Universität Nishnij Novgorod, Russland, 17.10.19.10.2001

Prof. Dr. Speranţa Stanescu, Universität Bukarest, Rumänien, 1.2.30.4.2001

Matt Stevens, Universität Oxford, Großbritannien, 16.7.-29.9.2001

Patrick Stevenson, Universität Southampton, England, 25.6.-29.6.2001

Mariana Trendafcheva, Universität Plovdiv, Bulgarien, 1.10.-31.7.2001

Dipl.-phil. Zorica Trpčevska, Universität Tetovo, Mazedonien, 20.4.5.7.2001 
Céline Vié, Universität Sorbonne, Paris, Frankreich, 1.10.-30.11.2001 Dr. phil. hab. Maja Volodina, Lomonosov Universität Moskau, Russland Erminka Zilić, Universität Sarajevo, Bosnien-Herzegowina, 4.5--17.5.2001 Prof. Dr. Jianhua Zhu, Tongji-Universität, Shanghai, VR China, 6.8.2001

\subsection{Praktika}

Ungeachtet der Tatsache, dass Praktika am IDS nicht vergütet werden können, ist ein wachsendes Interesse bei Studierenden an Praktika zu verzeichnen. In der Regel dauert ein Praktikum vier Wochen. Die Studierenden sollten die Zwischenprüfung in Sprachwissenschaft abgelegt haben. Die Tätigkeitsbereiche werden nach Interesse der Studierenden von der Arbeitsstelle Öffentlichkeitsarbeit zusammengestellt.

2001 waren als Praktikanten am IDS:

Christian Duis, 24.08.01-30.10.01 (academica); Robert Drehmann, 11.06.01-02.11.01 (Euro-Schule); Jan Eckhard, 03.09.01-28.09.01 (Universität Heidelberg); Serpil Ergun, 04.09.01-21.09.01 (Universität Istanbul); This Fetzer, 19.03.01-27.04.01 (Schweiz); Kerstin Groß, (Universität Freiburg) 05.06.01-29.06.01; Jasmin Christine Hambsch, 13.08.0107.09.01 (Universität Karlsruhe); Dagmar Herrmann, 23.07.01-31.08.01; Sarah Hitzler 26.02.01-06.04.01 (Universität Bielefeld); Irmtraud Kaiser, 04.07.01-26.07.01 (Universität Heidelberg); Stephanie Morris, 17.09.0112.10.01 (Universität Basel); Magdalena Muehlemann, 03.09.01-28.09.01 (Universität Heidelberg); Adam Polarz, 23.01.01-16.01.01; Kathia Samland; 19.03.01-06.04.01 (Universität Bremen); Karin Vorderstemann, 19.02.01-16.03.01 (Universität Freiburg); Stefanie Wagner, 13.04.014.09.01 (Universität Trier); Kerstin Zipproth, 19.02.01-16.03.01 (Universität Bamberg)

\subsection{Besuchergruppen}

Im Jahr 2001 waren 10 Besuchergruppen - zusammen rund 260 Personen Gäste im IDS (die Gastwissenschaftlerinnen und Gastwissenschaftler sowie Bibliotheksgäste nicht mitgezählt). Darunter waren 22 Deutschlehrerinnen aus 20 Ländern im Rahmen eines Seminars des Goethe-Instituts sowie Absolventen der Diplom- und Verwaltungsakademien Rhein-Neckar. Auch Schülerinnen und Schüler mehrerer Deutsch-Leistungskurse aus Mannheim, Heidelberg und Umgebung lernten die Erforschung der deutschen Sprache an Ort und Stelle kennen. Darüber hinaus informierten sich in- und ausländische Germanistik-Studierende von deutschen Universitäten im Rahmen ihrer Seminare über die Arbeit des IDS. Die Besuchergruppen erwartet ein möglichst auf ihre Interessen und Forschungsschwerpunkte zugeschnittenes Programm, das von einer allgemeinen Einführung in die Arbeit des IDS und von einer Bibliotheksführung eingerahmt wird. 


\section{Gremien des Instituts für Deutsche Sprache}

\subsection{Stiftungsrat}

Vorsitzender: Ltd. Min. Rat Dr. Klaus Herberger, Ministerium für Wissenschaft, Forschung und Kunst Baden-Württemberg

Stellvertreter: Reg. Dir. Dr. Manfred Pusch, Bundesministerium für Bildung, Wissenschaft, Forschung und Technologie

Peter Roschy, Freundeskreis des IDS - LR I Becker (bis Juni), LR I Kobsa (ab Juli), Auswärtiges Amt - Bürgermeister Dr. Peter Kurz, Stadt Mannheim - Prof. Dr. Heinrich Löffler, Universität Basel - Min. Rat Kaag, Ministerium für Wissenschaft, Forschung und Kunst Baden-Württemberg

\subsection{Wissenschaftlicher Beirat}

Vorsitzender: Prof. Dr. Heinrich Löffler, Universität Basel

Stellvertreter: Prof. Dr. Ludwig Eichinger, Universität Kiel (bis März)

Prof. Dr. Konrad Ehlich, Universität München - Prof. Dr. Peter Eisenberg, Universität Potsdam - Prof. Dr. Werner Eroms, Universität Passau - Prof. Dr. Cathrine Fabricius-Hansen, Universität Oslo - Prof. Dr. Hans Uszkoreit, Universität des Saarlandes - Prof. Dr. Peter Wiesinger, Universität Wien Prof. Dr. Norbert Richard Wolf, Universität Würzburg

\subsection{Direktor}

Prof. Dr. Gerhard Stickel

\subsection{Leitungskollegium}

Direktor: Prof. Dr. Gerhard Stickel - Abteilungsleiter: Prof. Dr. Werner Kallmeyer (Pragmatik) - Prof. Dr. Ulrike Haß-Zumkehr (Lexik) - Prof. Dr. Gisela Zifonun (Grammatik) - Mitarbeiterausschussvorsitzende: Dr. Annette Trabold (bis September), Dr. Kathrin Steyer (ab Oktober)

\subsection{Mitarbeiterausschuss}

(bis September) Vorsitzende: Dr. Annette Trabold - Stellvertreter: Prof. Dr. Bruno Strecker - Helmut Frosch - Ulrich Reitemeier - Eric Seubert - Dr. Kathrin Steyer - Dr. Wilfried Schütte

(ab Oktober) Vorsitzende: Dr. Kathrin Steyer - Stellvertreterin: Dr. Sabine Karl - Dr. Eva Breindl-Hiller - PD Dr. Thomas Spranz-Fogasy - Dr. Ulrich Waßner - Dr. Anja Lobenstein-Reichmann - Dr. Kristina Fischer-Hupe

\subsection{Betriebsrat}

\section{Vorsitzender: Claus Hoffmann}

Stellvertreter: Dr. Karl-Heinz Bausch

Stefanie Ablaß (bis 30.4.2001) - Jean-Christoph Clade - Dr. Edeltraud Winkler - Wolfgang Rathke (ab 1.5.2001) 


\subsection{Internationaler Wissenschaftlicher Rat (Stand: Dezember 2001)}

Professor Dr. Werner Abraham, Groningen, Niederlande - Professor Dr. Ulrich Ammon, Duisburg - Professor Dr. Gerhard Augst, Siegen - Professor Dr. Peter Bassola, Szeged, Ungarn - Professor Dr. Henning Bergenholtz, Arhus, Dänemark - Professor Dr. Anne Betten, Salzburg, Österreich - Professor Dr. Harald Burger, Zürich, Schweiz - Professor Dr. Dieter Cherubim, Göttingen - Professor Dr. Michael Clyne, Clayton, Australien - Professor Dr. Tomasz Czarnecki, Gdansk, Polen - Professor Dr. Martine Dalmas, Paris, Frankreich - Professor Dr. Miloje Dordjevic, Sarajevo, Bosnien-Herzegovina - Professor Dr. Dimitrij Dobrovol'skij, Moskau, Russland - Professor Dr. Martin Durrell, Manchester, Großbritannien - Professor Dr. Veronika Ehrich, Tübingen - Professor Dr. Ulla Fix, Leipzig - Professor Dr. Csaba Földes, Veszprem, Ungarn - Professor Dr. Franciszek Grucza, Warschau, Polen - Professor Dr. Walter Haas, Freiburg, Schweiz - Professor Dr. Hans Jürgen Heringer, Augsburg - Professor Dr. Regina Hessky, Budapest, Ungarn - Professor Dr. Rudolf Hoberg, Darmstadt - Professor Dr. Werner Holly, Chemnitz - Professor Dr. Ludwig Jäger, Aachen - Professor Dr. Alan Kirkness, Auckland, Neuseeland - Professor Dr. Gottfried Kolde, Genf, Schweiz - Professor Dr. Erwin Koller, Braga, Portugal - Professor Dr. Jarmo Korhonen, Helsinki, Finnland - Professor Dr. Oddleif Leirbukt, Bergen, Norwegen - Professor Dr. Karl Lepa, Tartu, Estland - Professor Dr. Angelika Linke, Zürich, Schweiz - Professor Dr. Thomas A. Lovik, East Lansing, USA - Professor Dr. René Metrich, Nancy, Frankreich - Prof. Dr. Eva Neuland, Wuppertal - Professor Dr. Henrik Nikula, Turku, Finnland - Professor Dr. Ernesta Raciené, Vilnius, Litauen - Professor Dr. Carlo Serra Borneto, Rom, Italien - Professor Dr. Marisa Siguan, Barcelona, Spanien - Professor Dr. Speranta Stanescu, Bukarest, Rumänien - Professor Dr. Eugeniusz Tomiczek, Breslau, Polen - Professor Dr. Livia Tonelli, Trieste, Italien - Professor Dr. Michael Townson, Dublin, Irland - Professor Dr. Vural Ülkü, Mersin, Türkei - Professor Dr. Sigurd Wichter, Göttingen - Professor Dr. M.I. Teresa Zurdo, Madrid, Spanien

\subsection{Beiräte}

Beirat Deutsches Fremdwörterbuch

Prof. Dr. Oskar Reichmann, Heidelberg - Dr. Heino Speer, Heidelberg

\section{Beirat Erklärende Synonymik}

Prof. Dr. Herbert Ernst Wiegand, Heidelberg - Prof. Dr. Peter Rolf Lutzeier, London - Prof. Dr. Georg Meggle, Leipzig 


\section{Besondere Nachrichten}

\subsection{Personalia}

Prof. Dr. Claudia Fraas ist zum 1. November 2001 auf eine C-3 Professur für Medienkommunikation der TU Chemnitz berufen worden.

Prof. Dr. Gerhard Stickel erhielt am 17. Oktober 2001 in einer Feierstunde des Staatsministeriums Baden-Württemberg das Bundesverdienstkreuz am Bande. Er wurde für seine Verdienste um die Entwicklung des Instituts für Deutsche Sprache zu einer im In- und Ausland als Forschungszentrum hoch geachteten Institution ausgezeichnet.

Außerdem wurde er in den „Beirat Sprache“ des Goethe-Institutes Inter Nationes berufen. Der „Beirat Sprache“ beschäftigt sich mit Projekten und Aufgaben der Abteilung Spracharbeit Ausland (Betreuung der Pädagogischen Verbindungsarbeit und der Sprachkursarbeit weltweit), sowie der Spracharbeit der Institute im Inland und der Sektion Forschung und Entwicklung.

Dr. Peter Wagener, Leiter des Deutschen Spracharchivs war von Juli 2000 bis Juli 2001 für einen Aufenthalt in den USA beurlaubt. Dort war er zunächst als Research Scholar am Max-Kade-Institut der Universität

Madison/Wisconsin tätig, arbeitete an der Modernisierung des Tonarchivs mit reichhaltigen Beständen deutscher Dialekte in den USA und setzte seine Arbeiten zum Sprachwandel fort. Im Frühjahrssemester 2001 war er als Max-Kade-Professor am German Department der Universität Madison/Wisconsin tätig und begründete daneben den Zweigverein Madison der Gesellschaft für deutsche Sprache, Wiesbaden.

PD Dr. Gereon Müller wurde in das Editorial Board des Journal of Comparative Germanic Linguistics aufgenommen.

Dr. Manfred Hellmann (Lexik), Dr. Ursula Hoberg (Grammatik), Dr. Irmtraud Jüttner (Lexik), Hilde Magis (Verwaltung) und Dr. Elke Tellenbach (Lexik) gingen im Laufe des Jahres 2001 in den Ruhestand.

\subsection{Vermischtes}

Mit dem Europäischen Tag der Sprachen am 26. September 2001 begann das Institut für Deutsche Sprache eine öffentliche Vortragsreihe: das Sprachforum. Die Referenten sind Mitarbeiterinnen und Mitarbeiter des IDS, die sich in ihren Vorträgen an ein sprachlich interessiertes Publikum wenden.

Im Jahr 2001 fanden folgende Vorträge statt:

- 26.09.2001, PD Dr. Hardarik Blühdorn, Lehren und Lernen von Fremdsprachen am Anfang des dritten Jahrtausends. Wohin geht der Weg?

- 05.12.2001, Dr. Heidrun Kämper, „Es ist doch nirgendwo so interessant wie heute und hier!" Sprache und Gegenwartsbewusstsein in der frühen Nachkriegszeit.

Ausführlichere Informationen sind im Internet unter http://www.ids-mannheim.de/org/sprachforum/foruml.html einsehbar. 
Seit September 2001 ist die Abteilung Grammatik in eine berufsbegleitende Weiterbildung zum Bereich „Online-Journalismus“ involviert, die von der Akademie für Weiterbildung der Universitäten Heidelberg und Mannheim e. V. angeboten wird. In Kooperation mit der Journalistin Dr. Katrin Bischl wird das texttechnologische und (hypertext)linguistische KnowHow, das in den Hypermedia-Projekten der Abteilung aufgebaut wurde, in die Berufsfelder Journalismus und Öffentlichkeitsarbeit transferiert, d.h. in handwerklich nutzbare Strategien und Leitlinien zur Gestaltung von Hypermedia-Texten umgesetzt. Von Seiten des IDS sind Prof. Dr. Gisela Zifonun (wissenschaftliche Leitung), Dr. Angelika Storrer und Michael Beißwenger M. A. (Dozenten) beteiligt; die Weiterbildung wird vom Ministerium für Wissenschaft, Forschung und Kunst Baden-Württemberg für 3 Jahre unterstützt; die Kurse finden in jährlichem Turnus statt (vgl. http://www.akademie-fuer-weiterbildung.de/).

Unter Beteiligung des Instituts für Deutsche Sprache fand vom 25. bis 27. Oktober 2001 die zweite Konferenz von Sprachakademien und zentralen Sprachinstituten europäischer Staaten statt, diesmal an der Accademia della Crusca in Florenz. Wie schon die erste Konferenz dieser Art im Dezember 2000 am IDS in Mannheim hatte sie das Thema ,Europäische Hochsprachen und europäische Mehrsprachigkeit“. Die Teilnehmer aus neun Staaten der Europäischen Union (B, D, DK, F, GB, I, NL, S, SF) beschlossen die „Mannheim-Florentiner Empfehlungen zur Förderung der europäischen Hochsprachen". Vereinbart wurde außerdem die Einrichtung einer ständigen Arbeitsgemeinschaft, die auch Vertreter von Sprachinstitutionen weiterer europäischer Länder aufnehmen soll.

Hans-Olaf Henkel, Präsident der Wissenschaftsgemeinschaft Gottfried Wilhelm Leibniz, besuchte im Dezember das Institut für Deutsche Sprache und informierte sich über aktuelle Forschungsprojekte. Das Institut für Deutsche Sprache gehört zusammen mit 77 anderen außeruniversitären Forschungs- und Serviceeinrichtungen für die Forschung zur Wissenschaftsgemeinschaft Gottfried Wilhelm Leibniz e. V. (WGL). Dies ist ein Zusammenschluß wissenschaftlicher Einrichtungen, die gemeinsam vom Bund und von den Ländern finanziert werden. Näheres unter: http://www.wgl.de

\section{Personalstärke, Anschrift, finanzielle Angaben}

\subsection{Personalstärke (Stand: 1.11.2001)}

Mitarbeiter (einschl. Teilzeit- und Projektmitarbeiter):

wissenschaftliche Angestellte:

(davon beurlaubt: 1)

Verwaltungs-/technische Angestellte:

Projekt-Mitarbeiter:

Insgesamt: 
9.2 Anschrift

Institut für Deutsche Sprache

R5, 6-13

D-68161 Mannheim

Postanschrift:

Postfach 101621

D-68016 Mannheim

Telefon (0621) 1581-0

Telefax (0621) 1581-200

E-Mail: vorstand@ids-mannheim.de

internet: http://www.ids-mannheim.de

9.3 Haushalt des Instituts im Berichtsjahr

Einnahmen:

Ministerium für Wissenschaft, Forschung und

$\begin{array}{lll}\text { Kunst Baden-Württemberg } & \text { DM } & 6.982 .000\end{array}$

Bundesministerium für Bildung und Forschung $\quad$ DM $\quad \mathbf{6 . 9 8 2 . 0 0 0}$

Stadt Mannheim

$\mathrm{DM} \quad 20.000$

eigene Einnahmen

DM $\quad 152.000$

DM $\quad 14.136 .000$

Ausgaben:

Personalausgaben

DM $\quad 11.362 .000$

Sachausgaben

Zuweisungen, Zuschüsse

DM $\quad 2.632 .000$

Investitionen

DM $\quad 22.000$

DM $\quad 120.000$

DM $\quad 14.136 .000$

Projektmittel:

Bundesministerium für Bildung und Forschung:

Personalmittel

$\begin{array}{ll}\mathrm{DM} & 162.000 \\ \mathrm{DM} & 162.000\end{array}$

Land Baden-Württemberg:

ALIGNMENT

Personalmittel

$\begin{array}{ll}\mathrm{DM} & 22.000 \\ \mathrm{DM} & 22.000\end{array}$

Referenz-Korpus

Personalmittel

DM $\quad 122.400$

Sachmittel

\begin{tabular}{lr} 
DM & 30.000 \\
\hline $\mathrm{DM}$ & 152.400
\end{tabular}


DFG:

Tendenzen der Lexikalisierung

kommunikativer Konzepte

Personalmittel

Sachmittel

DM $\quad 134.000$

DM $\quad 3.200$

DM $\quad 137.200$

Neue Medien in der Bildung

Textgrammatische Grundlagen

Für die Hypertextualisierung

Personalmittel

\begin{tabular}{lr} 
DM & 14.000 \\
DM & 2.000 \\
\hline DM & 16.000
\end{tabular}

Sachmittel

\begin{tabular}{ll} 
DM & 247.000 \\
\hline $\mathrm{DM}$ & 247.000
\end{tabular}

Sprachvariation als kommunikative

Praxis: „Sprachvariation deutsch-türkisch“

Personalmittel

\begin{tabular}{lr} 
DM & 64.500 \\
DM & 4.000 \\
\hline DM & 68.500
\end{tabular}

"Jugendkulturelle mediale Stile"

Personalmittel

\begin{tabular}{lr} 
DM & 64.500 \\
DM & 6.000 \\
\hline DM & 70.500
\end{tabular}

Summe der Projektmittel

\begin{tabular}{lr} 
DM & 875.600 \\
DM & 14.136 .000 \\
\hline DM & 15.011 .600
\end{tabular}

Institutioneller Haushalt

10. Veröffentlichungen im Jahre 2001

JAHRBÜCHER DES INSTITUTS FÜR DEUTSCHE SPRACHE

Verlag Walter de Gruyter, Berlin/New York

Redaktion: Franz Josef Berens

Stickel, Gerhard (Hg.): Neues und Fremdes im deutschen Wortschatz. Aktueller lexikalischer Wandel. Jahrbuch 2000 des Instituts für deutsche Sprache. VIII/388 S.

STUDIEN ZUR DEUTSCHEN SPRACHE

Forschungen des Instituts für Deutsche Sprache 
Hg. von Bruno Strecker, Reinhard Fiehler und Hartmut Schmidt Gunter Narr Verlag, Tübingen

Band 20: Wagner, Franc: Implizite sprachliche Diskriminierung als Sprechakt. Lexikalische Indikatoren impliziter Diskriminierung in Medientexten. $180 \mathrm{~S}$.

Band 21: Meng, Katharina: Russlanddeutsche Sprachbiografien. Untersuchungen zur sprachlichen Integration von Aussiedlerfamilien. $549 \mathrm{~S}$.

Band 23: Schilling, Marcel: Reden und Spielen. Die Kommunikation zwischen Trainern und Spielern im gehobenen Amateurfußball. $447 \mathrm{~S}$.

Band 24: Harras, Gisela (Hg.): Kommunikationsverben: Konzeptuelle Ordnung und semantische Repräsentation. 229 S.

DEUTSCHE SPRACHE

Im Auftrag des Instituts für Deutsche Sprache

hg. von Hans-Werner Eroms (Geschäftsführung), Gisela Harras, Heinrich Löffler, Gerhard Stickel und Gisela Zifonun

Redaktion: Franz Josef Berens

Jahrgang 2001: 4 Hefte

SPRACHREPORT

Informationen und Meinungen zur deutschen Sprache

$\mathrm{Hg}$. vom Institut für Deutsche Sprache

Jahrgang 2001: 4 Hefte

SPRACHREPORT Gesamtregister 1996 -2000. Titel und Autoren. Hg. vom Institut für Deutsche Sprache. 2001.

VERÖFFENTLICHUNGEN IM EIGENVERLAG amades - Arbeitspapiere und Materialien zur deutschen Sprache $\mathrm{Hg}$. vom Institut für Deutsche Sprache

Band 1/01: Fleischer, Holm: Wandlungen im Sprachgebrauch - Referenz und Pragmatik der Pronomen in ostdeutschen Zeitungskommentaren. Am Beispiel der Leipziger Volkszeitung vor, während und nach der „Wende“. $204 \mathrm{~S}$.

Band 2/01: Germanistik und Deutschunterricht in 16 Ländern. Berichte aus dem Internationalen Wissenschaftlichen Rat des IDS 1998-1999. 96 S.

Band 3/01: Zifonun, Gisela: Grammatik des Deutschen im europäischen Vergleich: Der Relativsatz. 104 S.

Band 4/01: Zifonun, Gisela: Grammatik des Deutschen im europäischen Vergleich: Das Pronomen Teil I. Überblick und Personalpronomen. 104 S. 